\title{
Pythagorean Dombi fuzzy graphs
}

\author{
Muhammad Akram ${ }^{1}$ (D) Jawaria Mohsan Dar $^{1}$. Sumera Naz $^{2}$
}

Received: 2 January 2019 / Accepted: 26 April 2019 / Published online: 23 May 2019

(c) The Author(s) 2019

\begin{abstract}
Pythagorean fuzzy graph, a broadly used extension of fuzzy and intuitionistic fuzzy graph, is helpful in representing structural relationships between several objects where the relation between these objects is vague, while the Dombi operators with operational parameters have excellent flexibility. Utilizing these two concepts, this research paper proposes the novel concept of Pythagorean Dombi fuzzy graphs (PDFGs). Basically, graph terminology is employed for introducing Pythagorean fuzzy analogs of various fundamental graphical ideas using Dombi operator. Further, under Pythagorean Dombi fuzzy environment, regular, totally regular, strongly regular and biregular graphs are defined with appropriate illustration and some of their crucial properties are examined. Meanwhile, the notion of edge regularity of PDFG is also initiated with substantial characteristics. Finally, a numerical example related to evaluation of appropriate ETL software for a business intelligence project is presented to better understand PDFGs.
\end{abstract}

Keywords Pythagorean fuzzy sets $\cdot \mathcal{T}$-norms and $\mathcal{T}$-conorms $\cdot$ Pythagorean Dombi fuzzy graphs $\cdot$ Regularity of Pythagorean Dombi fuzzy graphs

\section{Introduction}

In the past few years, many operators were initiated and the most important among them appeared in numerous monographs concerning with fuzzy logic. Particularly, it includes min-max, Einstein, Hamacher, Frank, product, Lukasiewicz, Azcel-Alsina and Dombi operators. The product and Einstein operators are the special cases of Hamacher operator. From practical perspectives, these parametrical families hold one's attention because by considering different value of parameter a distinct argument can be formed.

Zadeh [1] recommended to utilize the product and the minimum operator for defining fuzzy set. Hamacher [2] emphasised that by taking the solution of associativity operational equality, these operators can be created. Later, he

Muhammad Akram

m.akram@pucit.edu.pk

Jawaria Mohsan Dar

jiadar4@gmail.com

Sumera Naz

nsumeranaz@gmail.com

1 Department of Mathematics, University of the Punjab, New Campus, Lahore 4590, Pakistan

2 Department of Mathematics, Government College Women University, Faisalabad, Pakistan obtained the rational structure of disjunctive and conjunctive operators in accordance with Kuwagaki's results [3]. From that moment on, the researchers working in the field of fuzzy theory proposed a more general form, i.e., triangular norms (t-norms) and triangular conorms (t-conorms). Menger [4] introduced t-norms and t-conorms within probabilistic metric framework, where numbers are employed to narrate the distance between two objects of the space. Schweizer and Sklar [5] presented many axioms and results related to t-norms and $\mathrm{t}$-conorms that showed the rapid progress of this field. Furthermore, these norms are certified as standard models for union and intersection of fuzzy sets by Alsina et al. [6]. Several extensions and summarizations of beneficial outcomes of $\mathcal{T}$-operators for the similar cause can be seen in Klement et al. [7] and [8], respectively. In every fuzzy logic application, specifically fuzzy graph theory and decision-making procedures, Zadeh's min and max operators have been extensively applied. From experimental and theoretical point of view, other $\mathcal{T}$-operators may perform better in some cases, especially in decision-making problems, such as product operator may tend to choose over min operator [9]. One has to observe and examine the characteristics of $\mathcal{T}$-operators like suitability of the model, simplicity and implementation of hardware and software before the appropriate selection of these operators for a stated application. Since the study and work on 
these operators has broadened, multiple choices are available for choosing $\mathcal{T}$-operators that may be preferable for given research.

A pictorial representation that bonds the items together is known as 'graph'. But if in the bonding there occurs haziness, then the graph can be considered as fuzzy graph. Rosenfeld [10] established the layout of fuzzy graphs by taking into account fuzzy relations on fuzzy sets (FSs) with min and max operators. As the hesitancy part was not characterized explicitly, Atanassov [11] extended fuzzy sets to intuitionistic fuzzy sets (IFSs) by allocating membership $\mu$ and non-membership grade $v$ to the items, satisfying the constrain $\mu+v \leq 1$ with hesitancy part $\pi=1-\mu-v$. At that time graph theory was widely applied in almost every field of real life; hence Shannon and Atanassov [12] put forward the idea of intuitionistic fuzzy graphs by considering intuitionistic fuzzy relations on IFSs. Yager [13-15] inaugurated Pythagorean fuzzy sets (PFSs), a new extension of IFSs, to manage the complex uncertainty and impreciseness with constrain $\mu^{2}+v^{2} \leq 1$, where $\mu$ and $v$ represent membership and non-membership grade, respectively. Afterward, for explicating the dual features of an item, Zhang and $\mathrm{Xu}$ [16] proposed the notion of Pythagorean fuzzy number (PFN). The motivation of PFSs can be described as follows: in a decision-making environment, a specialist gives the preference information about an alternative with the membership grade 0.6 and the non-membership grade 0.5 ; it is noted that the intuitionistic fuzzy number (IFN) fails to address this situation, as $0.6+0.5>1$. But $(0.6)^{2}+(0.5)^{2} \leq 1$. Hence PFSs comprise and accommodate greater amount of vagueness than IFSs. The comparison between IFN space and PFN space is shown in Fig. 1. It has been successfully applied in numerous areas, including the internet stocks investment [16], the service quality of domestic airline [17] and the governor selection of the Asian Investment Bank [18]. In the practical multi-criteria group decision-making problems, Akram et al. [19] showed that PFS has much stronger ability to model fuzziness. Under PF environment, many researchers have initiated work in different directions and acquired various eminent results [17]. Some operations on PFSs [20] and Pythagorean fuzzy TODIM approach to multi-criteria decision making [18] have been discussed. Furthermore, the PFS has been investigated from different perspectives, including aggregation operators [21,22]. Garg [23-26] explored applications of Pythagorean fuzzy sets in decision-making problems. Graph theory has several applications, including cluster analysis and optimization of networks. Hence on the basis of its applications, Naz et al. [27] proffered the idea of Pythagorean fuzzy graphs (PFGs) using min and max operators and holding Pythagorean fuzzy relations on PFSs. Verma et al. [28] opened up the concept of strong Pythagorean fuzzy graphs and defined complement as well. Energy under Pythagorean fuzzy environment was

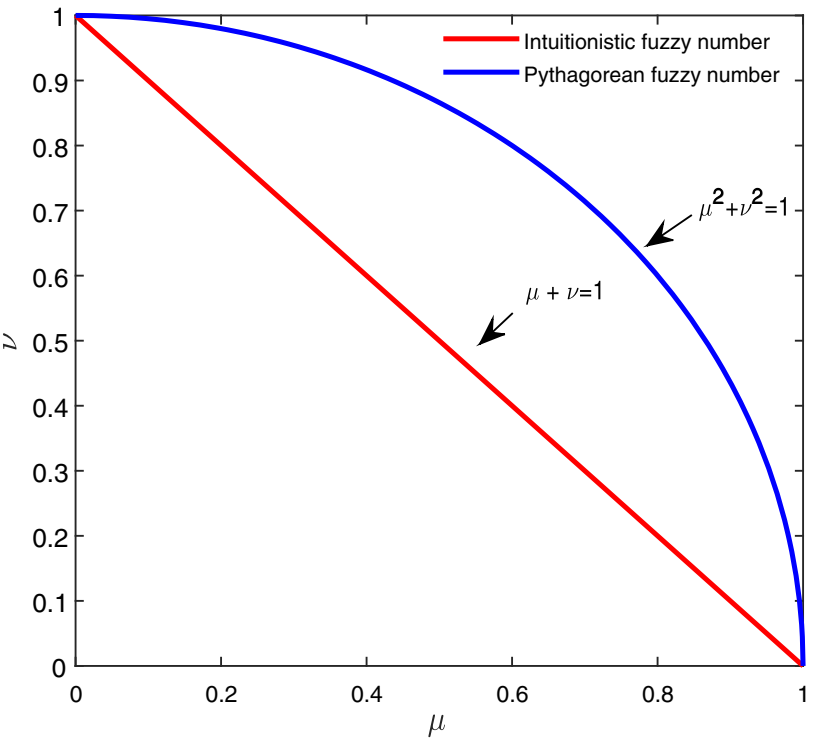

Fig. 1 Comparison of spaces of the IFN and the PFN

discussed by Akram and Naz [29]. Akram et al. [19,30-32] proposed certain graphs and explored their crucial properties under Pythagorean fuzzy circumstances. Recently, Akram and Habib [33] discussed regularity of $q$-rung picture fuzzy graphs with applications. Habib et al. [34] presented the notion of $q$-Rung orthopair fuzzy competition graphs by considering the most wide spread max and min operators and gave an application in the soil ecosystem.

Dombi [35] inaugurated Dombi operator with flexible operational parameter in 1982. This operator is exceptional as the sign of the parameter discovers whether the operator type is disjunctive or conjunctive. In decision-making problems, it is very useful as by taking distinct values of operational parameters, different arguments can be made depending upon the requirement or one's need. For this precedence, Chen and Ye [36], Jana et al. [37], Shi and Ye [38] used Dombi operations and presented MCDM problem in singlevalued neutrosophic, neutrosophic cubic and bipolar fuzzy environment, respectively. Liu et al. [39] proffered MCGDM problem utilizing Dombi Bonferroni mean operator on IFSs. He [40] investigated typhoon disaster assessment by considering Dombi operations in hesitant fuzzy environment. From the existing studies, it is observed that Dombi operational parameters have flexible nature in decision-making areas. Fuzzy graph theory can easily structure and model decision-making problems with uncertainty. A very limited effort is made for using Dombi operator in the field of graph theory. Hence on the basis of it, Ashraf et al. [41] presented the idea of Dombi fuzzy graph (DFG). As Pythagorean fuzzy graph is more powerful and more practical tool having the capability to deal with imprecise and incomplete information in different decision-making disciplines, such as engineer- 
ing, mathematics, statistics, artificial intelligence, medical and social sciences than fuzzy graphs. Therefore, the main objective of this research article was to emphasize on the fact that for generalizing the classical graphs to Pythagorean fuzzy graphs, the min and max operators are not preferable to model certain real-world situations. Further, our aim was to assemble the development introduced by Klement, Alsina, Hamachar and other founders in the field of Pythagorean fuzzy graph theory. The paper is accumulated to demonstrate the usage of a $\mathcal{T}$-operator, especially the Dombi operator. As Dombi operators with operational parameters, have excellent flexibility and have not yet been applied for Pythagorean fuzzy graphs, hence motivated from these operators, this paper introduces the notion of PDFGs as a generalization of Dombi fuzzy graphs. Further, some substantial characteristics like strongness, completeness, vertex and edge regularity are inspected as they are extensively applied in designing reliable computer networks and matrix representations.

The presented research article is structured as follows: Sect. 2 describes the basic notions and terminologies which will be utilized in the rest of sections. In Sect. 3, we propose the novel concept of Pythagorean Dombi fuzzy graphs and define the complement, homomorphism, isomorphism, completeness and strongness with appropriate illustration. In Sect. 4, we introduce regular, totally regular, strongly regular, biregular, edge regular, totally edge regular Pythagorean Dombi fuzzy graphs and examine some of their crucial characteristics. Section 5 presents a decision-making algorithm in Pythagorean Dombi fuzzy environment and solves a numerical example to illustrate the developed method. Section 6 contains concluding remarks and points out directions for future work.

\section{Preliminaries}

In this section, some prerequisite notations and terminologies have been stated for better understating.

In crisp sense, a graph is an ordered pair $G^{*}=(\mathscr{V}, E)$, where $\mathscr{V}$ is a vertex set and $E$ is the edge set of $G^{*}$. A vertex connected by an edge to a vertex $y$ is called a neighbor of $y$. The number of edges incident to a vertex $y$ is called the degree of that vertex. A graph without loops and multiple edges is called simple graph, whereas a graph in which each pair of graph vertices is connected by an edge is known as complete graph. The complement $\overline{G^{*}}$ of a graph, $G^{*}$, is a graph having vertex set same as in $G^{*}$, in which two vertices are incident if and only if they are not incident in $G^{*}$. If there occurs a one-one correspondence between the vertices of two graphs $G_{1}^{*}=\left(\mathscr{V}_{1}, E_{1}\right)=\left(\mathscr{V}\left(G_{1}^{*}\right), E\left(G_{1}^{*}\right)\right)$ and $G_{2}^{*}=\left(\mathscr{V}_{2}, E_{2}\right)=\left(\mathscr{V}\left(G_{2}^{*}\right), E\left(G_{2}^{*}\right)\right)$ which preserves adjacency, then the graphs $G_{1}^{*}$ and $G_{2}^{*}$ are called isomorphic. A self-complementary graph is a graph which is isomorphic to its complement. A regular and biregular graph is a graph, where each vertex has the same number of neighbors and each two vertices on the same side of the given bipartition have the same degree as each other, respectively. A graph with direction is called directed graph, where without direction is known as undirected graph.

Definition 1 [1] A fuzzy set (FS) on a universe $\mathscr{X}$ is an object of the following form:

$\mathscr{A}=\left\{\left\langle s, \mu_{\mathscr{A}}(s)\right\rangle \mid s \in \mathscr{X}\right\}$,

where $\mu_{\mathscr{A}}: \mathscr{X} \longrightarrow[0,1]$ represents the membership grades of $\mathscr{A}$.

Definition 2 [10] A fuzzy set on $\mathscr{X} \times \mathscr{X}$ is said to be a fuzzy relation $(\mathrm{FR})$ on $\mathscr{X}$, denoted by

$\mathscr{B}=\left\{\left\langle s t, \mu_{\mathscr{B}}(s t)\right\rangle \mid s t \in \mathscr{X} \times \mathscr{X}\right\}$,

where $\mu_{\mathscr{B}}: \mathscr{X} \times \mathscr{X} \longrightarrow[0,1]$ represents the membership grades of $\mathscr{A}$.

Definition 3 [10] A fuzzy graph on a non-empty set $\mathscr{X}$ is a pair $\mathscr{G}=(\mathscr{A}, \mathscr{B})$ with $\mathscr{A}$ a FS on $\mathscr{X}$ and $\mathscr{B}$ a FR on $\mathscr{X}$ such that

$\mu_{\mathscr{B}}(s t) \leq \mu_{\mathscr{A}}(s) \wedge \mu_{\mathscr{A}}(t)$

for all $s, t \in \mathscr{X}$, where $\mathscr{A}: \mathscr{X} \longrightarrow[0,1]$ and $\mathscr{B}:$ $\mathscr{X} \times \mathscr{X} \longrightarrow[0,1]$.

Definition 4 [11] An intuitionistic fuzzy set (IFS) on a universe $\mathscr{X}$ is an object of the form

$\mathscr{A}=\left\{\left\langle s, \mu_{\mathscr{A}}(s), v_{\mathscr{A}}(s)\right\rangle \mid s \in \mathscr{X}\right\}$,

where $\mu_{\mathscr{A}}: \mathscr{X} \longrightarrow[0,1]$ and $v_{\mathscr{A}}: \mathscr{X} \longrightarrow[0,1]$ represent the membership and non-membership grades of $\mathscr{A}$, and $\mu_{\mathscr{A}}, v_{\mathscr{A}}$ satisfies the condition $0 \leq \mu_{\mathscr{A}}(s)+v_{\mathscr{A}}(s) \leq 1$ for all $s \in \mathscr{X}$.

Definition 5 [12] An intuitionistic fuzzy set on $\mathscr{X} \times \mathscr{X}$ is said to be an intuitionistic fuzzy relation (IFR) on $\mathscr{X}$, denoted by

$\mathscr{B}=\left\{\left\langle s t, \mu_{\mathscr{B}}(s t), v_{\mathscr{B}}(s t)\right\rangle \mid s \in \mathscr{X}\right\}$,

where $\mu_{\mathscr{B}}: \mathscr{X} \times \mathscr{X} \longrightarrow[0,1]$ and $\nu_{\mathscr{B}}: \mathscr{X} \times \mathscr{X} \longrightarrow$ $[0,1]$ represent the membership and non-membership grades of $\mathscr{B}$, respectively, such that $0 \leq \mu_{\mathscr{B}}(s t)+v_{\mathscr{B}}(s t) \leq 1$ for all $s, t \in \mathscr{X}$. 
Definition 6 [12] An intuitionistic fuzzy graph (IFG) on a non-empty set $\mathscr{X}$ is a pair $\mathscr{G}=(\mathscr{A}, \mathscr{B})$ with $\mathscr{A}$ an IFS on $\mathscr{X}$ and $\mathscr{B}$ an IFR on $\mathscr{X}$ such that

$\mu_{\mathscr{B}}(s t) \leq \mu_{\mathscr{A}}(s) \wedge \mu_{\mathscr{A}}(t), v_{\mathscr{B}}(s t) \geq v_{\mathscr{A}}(s) \vee v_{\mathscr{A}}(t)$

and $0 \leq \mu_{\mathscr{B}}(s t)+v_{\mathscr{B}}(s t) \leq 1$ for all $s, t \in \mathscr{X}$, where $\mu_{\mathscr{B}}: \mathscr{X} \times \mathscr{X} \longrightarrow[0,1]$ and $\nu_{\mathscr{B}}: \mathscr{X} \times \mathscr{X} \longrightarrow[0,1]$ represent the membership and non-membership grades of $\mathscr{B}$, respectively.

Definition 7 [13] A Pythagorean fuzzy set (PFS) on a universe $\mathscr{X}$ is an object of the form

$\mathscr{A}=\left\{\left\langle s, \mu_{\mathscr{A}}(s), v_{\mathscr{A}}(s)\right\rangle \mid s \in \mathscr{X}\right\}$,

where $\mu_{\mathscr{A}}: \mathscr{X} \longrightarrow[0,1]$ and $v_{\mathscr{A}}: \mathscr{X} \longrightarrow[0,1]$ represent the membership and non-membership grades of $\mathscr{A}$, and $\mu_{\mathscr{A}}, v_{\mathscr{A}}$ satisfies the condition $0 \leq \mu_{\mathscr{A}}^{2}(s)+v_{\mathscr{A}}^{2}(s) \leq 1$ for all $s \in \mathscr{X}$.

Definition 8 [27] A Pythagorean fuzzy set on $\mathscr{X} \times \mathscr{X}$ is said to be a Pythagorean fuzzy relation (PFR) on $\mathscr{X}$, denoted by

$\mathscr{B}=\left\{\left\langle s t, \mu_{\mathscr{B}}(s t), v_{\mathscr{B}}(s t)\right\rangle \mid s \in \mathscr{X}\right\}$,

where $\mu_{\mathscr{B}}: \mathscr{X} \times \mathscr{X} \longrightarrow[0,1]$ and $\nu_{\mathscr{B}}: \mathscr{X} \times \mathscr{X} \longrightarrow$ $[0,1]$ represent the membership and non-membership grades of $\mathscr{B}$, respectively, such that $0 \leq \mu_{\mathscr{B}}^{2}(s t)+v_{\mathscr{B}}^{2}(s t) \leq 1$ for all $s, t \in \mathscr{X}$.

Definition 9 [27] A Pythagorean fuzzy graph (PFG) on a non-empty set $\mathscr{X}$ is a pair $\mathscr{G}=(\mathscr{A}, \mathscr{B})$ with $\mathscr{A}$ a PFS on $\mathscr{X}$ and $\mathscr{B}$ a PFR on $\mathscr{X}$ such that

$\mu_{\mathscr{B}}(s t) \leq \mu_{\mathscr{A}}(s) \wedge \mu_{\mathscr{A}}(t), v_{\mathscr{B}}(s t) \geq v_{\mathscr{A}}(s) \vee v_{\mathscr{A}}(t)$

and $0 \leq \mu_{\mathscr{B}}^{2}(s t)+v_{\mathscr{B}}^{2}(s t) \leq 1$ for all $s, t \in \mathscr{X}$, where $\mu_{\mathscr{B}}: \mathscr{X} \times \mathscr{X} \longrightarrow[0,1]$ and $v_{\mathscr{B}}: \mathscr{X} \times \mathscr{X} \longrightarrow[0,1]$ represent the membership and non-membership grades of $\mathscr{B}$, respectively.

Definition 10 [7] A binary function $\mathcal{T}:[0,1] \times[0,1] \rightarrow$ $[0,1]$ is known as t-norm if for all $s, t, u \in[0,1]$, it satisfies the following:

1. $\mathcal{T}(s, 1)=s$ (boundary condition or neutral property),

2. $\mathcal{T}(s, t)=\mathcal{T}(t, s)$ (commutativity),

3. $\mathcal{T}(s, \mathcal{T}(t, u))=\mathcal{T}(\mathcal{T}(s, t), u)$ (associativity),

4. $\mathcal{T}(s, t) \leq \mathcal{T}(u, v)$ if $s \leq u$ and $t \leq v$ (monotonicity).

Replacing 1 by 0 in condition 1 , we obtain the concept of dual t-conorm or s-norm.

There are the following common t-norms:
- The minimum operator $\mathscr{M}(s, t)=\min (s, t)$.

- The product operator $\mathscr{P}(s, t)=s t$.

- The Dombi's t-norm $\frac{1}{1}, \gamma>$

0.

$$
1+\left[\left(\frac{1-s}{s}\right)^{\gamma}+\left(\frac{1-t}{t}\right)^{\gamma}\right]^{\frac{1}{\gamma}}
$$

The corresponding $\mathrm{t}$-conorms are as follows:

- The maximum operator $\mathscr{M}^{*}(s, t)=\max (s, t)$.

- The probabilistic sum $\mathscr{P}^{*}(s, t)=s+t-s t$.

- The Dombi's t-conorm

1

$\gamma>0$.

$$
\overline{1+\left[\left(\frac{1-s}{s}\right)^{-\gamma}+\left(\frac{1-t}{t}\right)^{-\gamma}\right]^{\frac{1}{-\gamma}},}
$$

One more set of $\mathcal{T}$-operators is $\mathcal{T}(s, t)=\frac{s t}{s+t-s t}$ and $\mathscr{S}(s, t)=\frac{s+t-2 s t}{1-s t}$, which can be acquired by putting $\gamma=1$ in Dombi's t-norm and t-conorm. Additionally, $\mathscr{P}(s, t) \leq \frac{s t}{s+t-s t} \leq \mathscr{M}(s, t)$ and $\mathscr{M}^{*}(s, t) \leq$ $\frac{s+t-2 s t}{1-s t} \leq \mathscr{P}^{*}(s, t)$.

Definition 11 [41] A Dombi fuzzy graph on underlying set $\mathscr{V}$ is an ordered pair $G=(\mathscr{A}, \mathscr{B})$, where $\mathscr{A}: \mathscr{V} \longrightarrow[0,1]$ is a fuzzy subset in $\mathscr{V}$ and $\mathscr{B}: \mathscr{V} \times \mathscr{V} \longrightarrow[0,1]$ is a symmetric fuzzy relation on $\mathscr{A}$ such that

$\mu_{\mathscr{B}}(s t) \leq \frac{\mu_{\mathscr{A}}(s) \mu_{\mathscr{A}}(t)}{\mu_{\mathscr{A}}(s)+\mu_{\mathscr{A}}(t)-\mu_{\mathscr{A}}(s) \mu_{\mathscr{A}}(t)}$

for all $s, t \in \mathscr{V}$, where $\mu_{\mathscr{A}}$ and $\mu_{\mathscr{B}}$ represent the membership grades of $\mathscr{A}$ and $\mathscr{B}$, respectively.

Definition 12 [27] A Pythagorean fuzzy preference relation (PFPR) on the set of alternatives $\mathscr{X}=\left\{x_{1}, x_{2}, \ldots, x_{n}\right\}$ is represented by a matrix $\mathscr{Q}=\left(r_{i j}\right)_{n \times n}$, where $r_{i j}=$ $\left(x_{i} x_{j}, \mu\left(x_{i} x_{j}\right), v\left(x_{i} x_{j}\right)\right)$ for all $i, j=1,2, \ldots, n$. For convenience, let $r_{i j}=\left(\mu_{i j}, v_{i j}\right)$ where $\mu_{i j}$ indicates the degree to which the object $x_{i}$ is preferred to the object $x_{j}, v_{i j}$ denotes the degree to which the object $x_{i}$ is not preferred to the object $x_{j}$ and $\pi_{i j}=\sqrt{1-\mu_{i j}^{2}-v_{i j}^{2}}$ is interpreted as a hesitancy degree, with the following conditions:

$$
\begin{aligned}
\mu_{i j}, v_{i j} & \in[0,1], \mu_{i j}^{2}+v_{i j}^{2} \leq 1, \\
\mu_{i j} & =v_{j i}, \mu_{i i}=v_{i i}=0.5, \text { for all } i, j=1,2, \ldots, n .
\end{aligned}
$$

For other applications and prerequisite terminologies, the readers are referred to [42-48]. 


\section{Pythagorean Dombi fuzzy graphs}

Definition 13 A Pythagorean Dombi fuzzy graph (PDFG) on underlying set $\mathscr{V}$ is an ordered pair $G=(\mathscr{A}, \mathscr{B})$, where $\mathscr{A}=\left(\mu_{\mathscr{A}}, v_{\mathscr{A}}\right): \mathscr{V} \longrightarrow[0,1]$ is a Pythagorean fuzzy subset in $\mathscr{V}$ and $\mathscr{B}=\left(\mu_{\mathscr{B}}, v_{\mathscr{B}}\right): \mathscr{V} \times \mathscr{V} \longrightarrow[0,1]$ is a symmetric Pythagorean fuzzy relation on $\mathscr{A}$ such that

$\mu_{\mathscr{B}}(s t) \leq \frac{\mu_{\mathscr{A}}(s) \mu_{\mathscr{A}}(t)}{\mu_{\mathscr{A}}(s)+\mu_{\mathscr{A}}(t)-\mu_{\mathscr{A}}(s) \mu_{\mathscr{A}}(t)}$,

$v_{\mathscr{B}}(s t) \leq \frac{v_{\mathscr{A}}(s)+v_{\mathscr{A}}(t)-2 v_{\mathscr{A}}(s) v_{\mathscr{A}}(t)}{1-v_{\mathscr{A}}(s) v_{\mathscr{A}}(t)}$

and $0 \leq \mu_{\mathscr{B}}^{2}(s t)+v_{\mathscr{B}}^{2}(s t) \leq 1$ for all $s, t \in \mathscr{V}$, where $\mu_{\mathscr{B}}$ and $\nu_{\mathscr{B}}$ represent the membership and non-membership grades of $\mathscr{B}$, respectively.

Remark 1 We call $\mathscr{A}$ the Pythagorean Dombi fuzzy vertex set of $G$ and $\mathscr{B}$ the Pythagorean Dombi fuzzy edge set of $G$.

- If $\mathscr{B}$ is symmetric on $\mathscr{A}$, then $G=(\mathscr{A}, \mathscr{B})$ is called PDFG.

- If $\mathscr{B}$ is not symmetric on $\mathscr{A}$, then $\mathcal{D}=(\mathscr{A}, \overrightarrow{\mathscr{B}})$ is called Pythagorean Dombi fuzzy digraph.

Example 1 Consider a graph $G^{*}=(\mathscr{V}, E)$, where $\mathscr{V}=$ $\left\{t_{1}, t_{2}, t_{3}, t_{4}, t_{5}, t_{6}\right\}$ and $E=\left\{t_{1} t_{2}, t_{1} t_{4}, t_{1} t_{6}, t_{2} t_{3}, t_{2} t_{5}, t_{3} t_{6}\right.$, $\left.t_{3} t_{4}, t_{4} t_{5}, t_{5} t_{6}\right\}$. Let $\mathscr{A}$ and $\mathscr{B}$ be Pythagorean Dombi fuzzy vertex set and Pythagorean Dombi fuzzy edge set defined on $\mathscr{V}$ and $E$, respectively.

$$
\begin{aligned}
\mathscr{A}= & \left\langle\left(\frac{t_{1}}{0.50}, \frac{t_{2}}{0.60}, \frac{t_{3}}{0.80}, \frac{t_{4}}{0.90}, \frac{t_{5}}{0.50}, \frac{t_{6}}{0.20}\right),\right. \\
& \left.\left(\frac{t_{1}}{0.60}, \frac{t_{2}}{0.70}, \frac{t_{3}}{0.40}, \frac{t_{4}}{0.20}, \frac{t_{5}}{0.70}, \frac{t_{6}}{0.90}\right)\right\rangle \text { and } \\
\mathscr{B}= & \left\langle\left(\frac{t_{1} t_{2}}{0.35}, \frac{t_{1} t_{4}}{0.45}, \frac{t_{1} t_{6}}{0.15}, \frac{t_{2} t_{3}}{0.50}, \frac{t_{2} t_{5}}{0.30}, \frac{t_{3} t_{6}}{0.15}, \frac{t_{3} t_{4}}{0.70}, \frac{t_{4} t_{5}}{0.40}, \frac{t_{5} t_{6}}{0.14}\right),\right. \\
& \left.\left(\frac{t_{1} t_{2}}{0.74}, \frac{t_{1} t_{4}}{0.61}, \frac{t_{1} t_{6}}{0.91}, \frac{t_{2} t_{3}}{0.72}, \frac{t_{2} t_{5}}{0.80}, \frac{t_{3} t_{6}}{0.91}, \frac{t_{3} t_{4}}{0.45}, \frac{t_{4} t_{5}}{0.71}, \frac{t_{5} t_{6}}{0.90}\right)\right\rangle .
\end{aligned}
$$

By routine computations, one can see from Fig. 2 that $G=$ $(\mathscr{A}, \mathscr{B})$ is a PDFG.

Definition 14 Let $\mathscr{B}=\left\{\left(s t, \mu_{\mathscr{B}}(s t), \nu_{\mathscr{B}}(s t)\right) \mid s t \in E\right\}$ be a Pythagorean Dombi fuzzy edge set in PDFG $G$; then

- The order of $G$ is symbolized by $\mathscr{O}(G)$ and given by $\mathscr{O}(G)=\left(\sum_{t \in \mathscr{V}} \mu_{\mathscr{A}}(t), \sum_{t \in \mathscr{V}} v_{\mathscr{A}}(t)\right)$.

- The size of $G$ is symbolized by $\mathscr{S}(G)$ and given by $\mathscr{S}(G)=\left(\sum_{s t \in E} \mu_{\mathscr{B}}(s t), \sum_{s t \in E} v_{\mathscr{B}}(s t)\right)$.

Example 2 From Example 1, we have

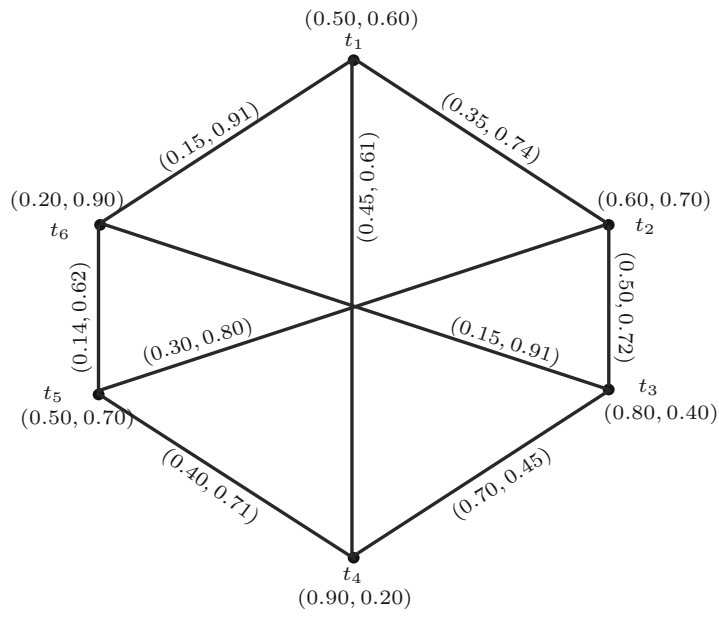

Fig. 2 Pythagorean Dombi fuzzy graph

- The order of $G \mathscr{O}(G)=\left(\sum_{t \in \mathscr{V}} \mu_{\mathscr{A}}(t), \sum_{t \in \mathscr{V}} \nu_{\mathscr{A}}(t)\right)=$ $(3.5,3.5)$.

- The size of $G \mathscr{S}(G)=\left(\sum_{s t \in E} \mu_{\mathscr{B}}(s t), \sum_{s t \in E} v_{\mathscr{B}}(s t)\right)$ $=(3.14,6.75)$.

Definition 15 Let $\mathscr{B}=\left\{\left(s t, \mu_{\mathscr{B}}(s t), \nu_{\mathscr{B}}(s t)\right) \mid s t \in E\right\}$ be a Pythagorean Dombi fuzzy edge set in PDFG $G$; then

- The degree of vertex $s \in \mathscr{V}$ is symbolized by $(\mathscr{D})_{G}(s)$ and defined by $(\mathscr{D})_{G}(s)=\left((\mathscr{D})_{\mu}(s),(\mathscr{D})_{\nu}(s)\right)$, where

$$
\begin{aligned}
(\mathscr{D})_{\mu}(s) & =\sum_{s, t \neq s \in \mathscr{V}} \mu_{\mathscr{B}}(s t) \\
& =\sum_{s, t \neq s \in \mathscr{V}} \frac{\mu_{\mathscr{A}}(s) \mu_{\mathscr{A}}(t)}{\mu_{\mathscr{A}}(s)+\mu_{\mathscr{A}}(t)-\mu_{\mathscr{A}}(s) \mu_{\mathscr{A}}(t)}, \\
(\mathscr{D})_{v}(s) & =\sum_{s, t \neq s \in \mathscr{V}} v_{\mathscr{B}}(s t) \\
& =\sum_{s, t \neq s \in \mathscr{V}} \frac{v_{\mathscr{A}}(s)+v_{\mathscr{A}}(t)-2 v_{\mathscr{A}}(s) v_{\mathscr{A}}(t)}{1-v_{\mathscr{A}}(s) v_{\mathscr{A}}(t)} .
\end{aligned}
$$

- The total degree of vertex $s \in \mathscr{V}$ is symbolized by $(\mathscr{T} \mathscr{D})_{G}(s)$ and defined by $(\mathscr{T} \mathscr{D})_{G}(s)=\left((\mathscr{T} \mathscr{D})_{\mu}(s)\right.$, $\left.(\mathscr{T} \mathscr{D})_{v}(s)\right)$, where

$$
\begin{aligned}
(\mathscr{T} \mathscr{D})_{\mu}(s)= & \sum_{s, t \neq s \in \mathscr{V}} \mu_{\mathscr{B}}(s t)+\mu_{\mathscr{A}}(s) \\
= & \sum_{s, t \neq s \in \mathscr{V}} \frac{\mu_{\mathscr{A}}(s) \mu_{\mathscr{A}}(t)}{\mu_{\mathscr{A}}(s)+\mu_{\mathscr{A}}(t)-\mu_{\mathscr{A}}(s) \mu_{\mathscr{A}}(t)} \\
& +\mu_{\mathscr{A}}(s), \\
(\mathscr{T} \mathscr{D})_{v}(s)= & \sum_{s, t \neq s \in \mathscr{V}} v_{\mathscr{B}}(s t)+v_{\mathscr{A}}(s)
\end{aligned}
$$




$$
\begin{aligned}
= & \sum_{s, t \neq s \in \mathscr{V}} \frac{v_{\mathscr{A}}(s)+v_{\mathscr{A}}(t)-2 v_{\mathscr{A}}(s) v_{\mathscr{A}}(t)}{1-v_{\mathscr{A}}(s) v_{\mathscr{A}}(t)} \\
& +v_{\mathscr{A}}(s) .
\end{aligned}
$$

Example 3 From Example 1, we have

- The degree of the vertices in $G$ are as follows:

$(\mathscr{D})_{G}\left(t_{1}\right)=(0.95,2.26),(\mathscr{D})_{G}\left(t_{2}\right)=(1.15,2.26)$,

$(\mathscr{D})_{G}\left(t_{3}\right)=(1.35,2.08),(\mathscr{D})_{G}\left(t_{4}\right)=(1.55,1.77)$,

$(\mathscr{D})_{G}\left(t_{5}\right)=(0.84,2.41),(\mathscr{D})_{G}\left(t_{6}\right)=(0.44,2.72)$.

- The total degree of the vertices in $G$ are as follows:

$(\mathscr{T} \mathscr{D})_{G}\left(t_{1}\right)=(1.45,2.86),(\mathscr{T} \mathscr{D})_{G}\left(t_{2}\right)=(1.75,2.96)$,

$(\mathscr{T} \mathscr{D})_{G}\left(t_{3}\right)=(2.15,2.48),(\mathscr{T} \mathscr{D})_{G}\left(t_{4}\right)=(2.45,1.97)$,

$(\mathscr{T} \mathscr{D})_{G}\left(t_{5}\right)=(1.34,3.11),(\mathscr{T} \mathscr{D})_{G}\left(t_{6}\right)=(0.64,3.62)$.

Definition 16 The complement of a PDFG $G=(\mathscr{A}, \mathscr{B})$ on an underlying graph $G^{*}=(\mathscr{V}, E)$ is a PDFG $\bar{G}=(\overline{\mathscr{A}}, \overline{\mathscr{B}})$ which is defined by

1. $\overline{\mu_{\mathscr{A}}(s)}=\mu_{\mathscr{A}}(s)$ and $\overline{v_{\mathscr{A}}(s)}=v_{\mathscr{A}}(s)$.

2. $\overline{\mu_{\mathscr{B}}(s t)}= \begin{cases}\frac{\mu_{\mathscr{A}}(s) \mu_{\mathscr{A}}(t)}{\mu_{\mathscr{A}}(s)+\mu_{\mathscr{A}}(t)-\mu_{\mathscr{A}}(s) \mu_{\mathscr{A}}(t)}, & \text { if } \mu_{\mathscr{B}}(s t)=0, \\ \frac{\mu_{\mathscr{A}}(s) \mu_{\mathscr{A}}(t)}{\mu_{\mathscr{A}}(s)+\mu_{\mathscr{A}}(t)-\mu_{\mathscr{A}}(s) \mu_{\mathscr{A}}(t)}-\mu_{\mathscr{B}}(s t), & \text { if } 0<\mu_{\mathscr{B}}(s t) \leq 1 .\end{cases}$

$$
\overline{v_{\mathscr{B}}(s t)}= \begin{cases}\frac{v_{\mathscr{A}}(s)+v_{\mathscr{A}}(t)-2 v_{\mathscr{A}}(s) v_{\mathscr{A}}(t)}{1-v_{\mathscr{A}}(s) v_{\mathscr{A}}(t)}, & \text { if } v_{\mathscr{B}}(s t)=0, \\ \frac{v_{\mathscr{A}}(s)+v_{\mathscr{A}}(t)-2 v_{\mathscr{A}}(s) v_{\mathscr{A}}(t)}{1-v_{\mathscr{A}}(s) v_{\mathscr{A}}(t)}-v_{\mathscr{B}}(s t), & \text { if } 0<v_{\mathscr{B}}(s t) \leq 1 .\end{cases}
$$

Example 4 Consider a PDFG $G$ over $\mathscr{V}=\left\{t_{1}, t_{2}, t_{3}, t_{4}\right\}$ as displayed in Fig. 3 and defined by

$$
\begin{aligned}
\mathscr{A}= & \left\langle\left(\frac{t_{1}}{0.40}, \frac{t_{2}}{0.20}, \frac{t_{3}}{0.90}, \frac{t_{4}}{0.30}\right),\right. \\
& \left.\left(\frac{t_{1}}{0.70}, \frac{t_{2}}{0.90}, \frac{t_{3}}{0.30}, \frac{t_{4}}{0.80}\right)\right\rangle \text { and } \\
\mathscr{B}= & \left\langle\left(\frac{t_{1} t_{2}}{0.13}, \frac{t_{1} t_{3}}{0.35}, \frac{t_{1} t_{4}}{0.20}, \frac{t_{2} t_{3}}{0.15}, \frac{t_{3} t_{4}}{0.25}\right),\right. \\
& \left.\left(\frac{t_{1} t_{2}}{0.91}, \frac{t_{1} t_{3}}{0.72}, \frac{t_{1} t_{4}}{0.83}, \frac{t_{2} t_{3}}{0.85}, \frac{t_{3} t_{4}}{0.80}\right)\right\rangle .
\end{aligned}
$$

By using Definition 16, one can obtain complement of PDFG as presented in Fig. 4 and defined by

$$
\begin{aligned}
\mathscr{A}= & \left\langle\left(\frac{t_{1}}{0.40}, \frac{t_{2}}{0.20}, \frac{t_{3}}{0.90}, \frac{t_{4}}{0.30}\right),\right. \\
& \left.\left(\frac{t_{1}}{0.70}, \frac{t_{2}}{0.90}, \frac{t_{3}}{0.30}, \frac{t_{4}}{0.80}\right)\right\rangle \text { and }
\end{aligned}
$$
$(\overline{\mathscr{A}}, \overline{\mathscr{B}})$ is a PDFG. plement of PDFG, we have

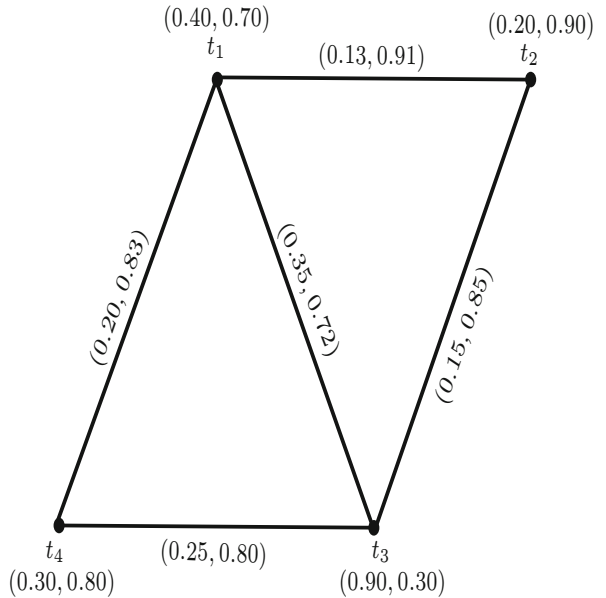

Fig. 3 Pythagorean Dombi fuzzy graph

$\mathscr{B}=\left\langle\left(\frac{t_{1} t_{2}}{0.02}, \frac{t_{1} t_{3}}{0.03}, \frac{t_{1} t_{4}}{0.01}, \frac{t_{2} t_{3}}{0.05}, \frac{t_{3} t_{4}}{0.04}, \frac{t_{2} t_{4}}{0.14}\right)\right.$,

By routine computations, one can see from Fig. 4 that $\bar{G}=$

Theorem 1 If $G=(\mathscr{A}, \mathscr{B})$ is a PDFG, then $\overline{\bar{G}}=G$.

Proof Suppose that $G$ is a PDFG. Then by definition of com-

$$
\begin{aligned}
\overline{\overline{\mu_{\mathscr{A}}(s)}} & =\overline{\mu_{\mathscr{A}}(s)}=\mu_{\mathscr{A}}(s) \text { and } \overline{\overline{v_{\mathscr{A}}(s)}} \\
& =\overline{v_{\mathscr{A}}(s)}=v_{\mathscr{A}}(s) \text { for all } \mathrm{s} \in \mathscr{V} .
\end{aligned}
$$

If $\mu_{\mathscr{B}}(s t)=0$ and $v_{\mathscr{B}}(s t)=0$, then

$$
\begin{aligned}
& \overline{\overline{\mu_{\mathscr{B}}(s t)}}=\frac{\overline{\mu_{\mathscr{A}}(s) \mu_{\mathscr{A}}(t)}}{\overline{\mu_{\mathscr{A}}(s)}+\overline{\mu_{\mathscr{A}}(t)}-\overline{\mu_{\mathscr{A}}(s) \mu_{\mathscr{A}}(t)}} \\
& =\frac{\mu_{\mathscr{A}}(s) \mu_{\mathscr{A}}(t)}{\mu_{\mathscr{A}}(s)+\mu_{\mathscr{A}}(t)-\mu_{\mathscr{A}}(s) \mu_{\mathscr{A}}(t)}=\mu_{\mathscr{B}}(s t),
\end{aligned}
$$




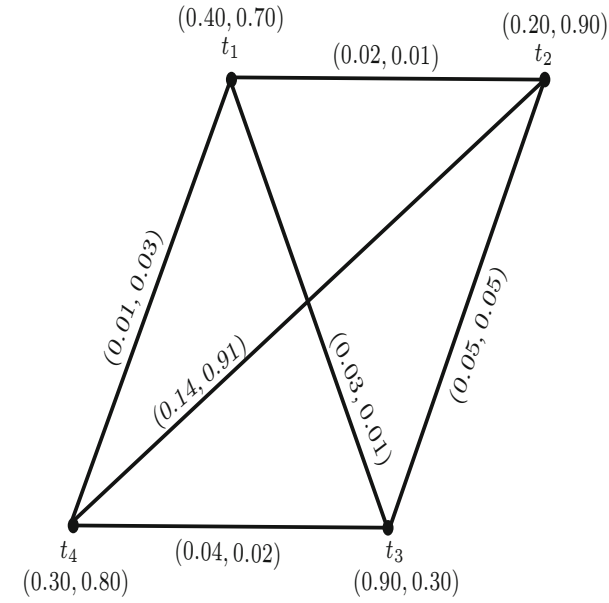

Fig. 4 Complement of Pythagorean Dombi fuzzy graph

$$
\begin{aligned}
\overline{\overline{v_{\mathscr{B}}(s t)}} & =\frac{\overline{v_{\mathscr{A}}(s)}+\overline{v_{\mathscr{A}}(t)}-2 \overline{v_{\mathscr{A}}(s) v_{\mathscr{A}}(t)}}{1-\overline{v_{\mathscr{A}}(s) v_{\mathscr{A}}(t)}} \\
& =\frac{v_{\mathscr{A}}(s)+v_{\mathscr{A}}(t)-2 v_{\mathscr{A}}(s) v_{\mathscr{A}}(t)}{1-v_{\mathscr{A}}(s) v_{\mathscr{A}}(t)}=v_{\mathscr{B}}(s t) .
\end{aligned}
$$

If $0<\mu_{\mathscr{B}}(s t), v_{\mathscr{B}}(s t) \leq 1$, then

$$
\begin{aligned}
& \overline{\overline{\mu_{\mathscr{B}}(s t)}}=\frac{\overline{\mu_{\mathscr{A}}(s) \mu_{\mathscr{A}}(t)}}{\overline{\mu_{\mathscr{A}}(s)}+\overline{\mu_{\mathscr{A}}(t)}-\overline{\mu_{\mathscr{A}}(s) \mu_{\mathscr{A}}(t)}}-\overline{\mu_{\mathscr{B}}(s t)} \\
& =\frac{\mu_{\mathscr{A}}(s) \mu_{\mathscr{A}}(t)}{\mu_{\mathscr{A}}(s)+\mu_{\mathscr{A}}(t)-\mu_{\mathscr{A}}(s) \mu_{\mathscr{A}}(t)} \\
& -\left(\frac{\mu_{\mathscr{A}}(s) \mu_{\mathscr{A}}(t)}{\mu_{\mathscr{A}}(s)+\mu_{\mathscr{A}}(t)-\mu_{\mathscr{A}}(s) \mu_{\mathscr{A}}(t)}-\mu_{\mathscr{B}}(s t)\right) \\
& =\mu_{\mathscr{B}}(s t) \text {, } \\
& \overline{\overline{v_{\mathscr{B}}(s t)}}=\frac{\overline{v_{\mathscr{A}}(s)}+\overline{v_{\mathscr{A}}(t)}-2 \overline{v_{\mathscr{A}}(s) v_{\mathscr{A}}(t)}}{1-\overline{v_{\mathscr{A}}(s) v_{\mathscr{A}}(t)}}-\overline{v_{\mathscr{B}}(s t)} \\
& =\frac{v_{\mathscr{A}}(s)+v_{\mathscr{A}}(t)-2 v_{\mathscr{A}}(s) v_{\mathscr{A}}(t)}{1-v_{\mathscr{A}}(s) v_{\mathscr{A}}(t)} \\
& -\left(\frac{v_{\mathscr{A}}(s)+v_{\mathscr{A}}(t)-2 v_{\mathscr{A}}(s) v_{\mathscr{A}}(t)}{1-v_{\mathscr{A}}(s) v_{\mathscr{A}}(t)}-v_{\mathscr{B}}(s t)\right) \\
& =v_{\mathscr{B}}(s t)
\end{aligned}
$$

for all $s, t \in \mathscr{V}$. Hence the complement of a complement PDFG is a PDFG itself.

Definition 17 A homomorphism $\mathscr{H}: G_{1} \rightarrow G_{2}$ of two PDFGs $G_{1}=\left(\mathscr{A}_{1}, \mathscr{B}_{1}\right)$ and $G_{2}=\left(\mathscr{A}_{2}, \mathscr{B}_{2}\right)$ is a mapping $\mathscr{H}: \mathscr{V}_{1} \rightarrow \mathscr{V}_{2}$ satisfying

1. $\mu_{\mathscr{A}_{1}}(s) \leq \mu_{\mathscr{A}_{2}}(\mathscr{H}(s)), v_{\mathscr{A}_{1}}(s) \leq v_{\mathscr{A}_{2}}(\mathscr{H}(s))$,

2. $\mu_{\mathscr{B}_{1}}(s t) \leq \mu_{\mathscr{B}_{2}}(\mathscr{H}(s) \mathscr{H}(t)), v_{\mathscr{B}_{1}}(s t) \leq v_{\mathscr{B}_{2}}(\mathscr{H}(r)$ $\mathscr{H}(s))$ for all $s \in \mathscr{V}_{1}, s t \in E_{1}$.
Definition 18 An isomorphism $\mathscr{H}: G_{1} \rightarrow G_{2}$ of two PDFGs $G_{1}=\left(\mathscr{A}_{1}, \mathscr{B}_{1}\right)$ and $G_{2}=\left(\mathscr{A}_{2}, \mathscr{B}_{2}\right)$ is a bijective mapping $\mathscr{H}: \mathscr{V}_{1} \rightarrow \mathscr{V}_{2}$ satisfying

1. $\mu_{\mathscr{A}_{1}}(s)=\mu_{\mathscr{A}_{2}}(\mathscr{H}(s)), v_{\mathscr{A}_{1}}(s)=v_{\mathscr{A}_{2}}(\mathscr{H}(s))$,

2. $\mu_{\mathscr{B}_{1}}(s t)=\mu_{\mathscr{B}_{2}}(\mathscr{H}(s) \mathscr{H}(t)), v_{\mathscr{B}_{1}}(s t)=v_{\mathscr{B}_{2}}(\mathscr{H}(s) \mathscr{H}$ $(t))$ for all $s \in \mathscr{V}_{1}$, st $\in E_{1}$.

Definition 19 A weak isomorphism $\mathscr{H}: G_{1} \rightarrow G_{2}$ of two PDFGs $G_{1}=\left(\mathscr{A}_{1}, \mathscr{B}_{1}\right)$ and $G_{2}=\left(\mathscr{A}_{2}, \mathscr{B}_{2}\right)$ is a bijective mapping $\mathscr{H}: \mathscr{V}_{1} \rightarrow \mathscr{V}_{2}$ satisfying

1. $\mathscr{H}$ is a homomorphism,

2. $\mu_{\mathscr{A}_{1}}(s)=\mu_{\mathscr{A}_{2}}(\mathscr{H}(s)), v_{\mathscr{A}_{1}}(s)=v_{\mathscr{A}_{2}}(\mathscr{H}(s))$ for all $s$ $\in \mathscr{V}_{1}$.

Definition 20 A co-weak isomorphism $\mathscr{H}: G_{1} \rightarrow G_{2}$ of two PDFGs $G_{1}=\left(\mathscr{A}_{1}, \mathscr{B}_{1}\right)$ and $G_{2}=\left(\mathscr{A}_{2}, \mathscr{B}_{2}\right)$ is a bijective mapping $\mathscr{H}: \mathscr{V}_{1} \rightarrow \mathscr{V}_{2}$ satisfying

1. $\mathscr{H}$ is a homomorphism,

2. $\mu_{\mathscr{B}_{1}}(s t)=\mu_{\mathscr{B}_{2}}(\mathscr{H}(s) \mathscr{H}(t)), v_{\mathscr{B}_{1}}(s t)=v_{\mathscr{B}_{2}}(\mathscr{H}(s)$ $\mathscr{H}(t))$ for all $s t \in E_{1}$.

Definition 21 A PDFG $G=(\mathscr{A}, \mathscr{B})$ is called selfcomplementary if $\bar{G} \cong G$.

Proposition 1 If $G=(\mathscr{A}, \mathscr{B})$ is a self-complementary PDFG, then

$$
\begin{aligned}
\sum_{s \neq t} \mu_{\mathscr{B}}(s t) & =\frac{1}{2} \sum_{s \neq t} \frac{\mu_{\mathscr{A}}(s) \mu_{\mathscr{A}}(t)}{\mu_{\mathscr{A}}(s)+\mu_{\mathscr{A}}(t)-\mu_{\mathscr{A}}(s) \mu_{\mathscr{A}}(t)} \\
\sum_{s \neq t} v_{\mathscr{B}}(s t) & =\frac{1}{2} \sum_{s \neq t} \frac{v_{\mathscr{A}}(s)+v_{\mathscr{A}}(t)-2 v_{\mathscr{A}}(s) v_{\mathscr{A}}(t)}{1-v_{\mathscr{A}}(s) v_{\mathscr{A}}(t)}
\end{aligned}
$$

Proof Assume that $G$ is a self-complementary PDFG; then there occurs an isomorphism $\mathscr{H}: \mathscr{V} \longrightarrow \mathscr{V}$ such that

$$
\overline{\mu_{\mathscr{A}}(\mathscr{H}(s))}=\mu_{\mathscr{A}}(s), \quad \overline{v_{\mathscr{A}}(\mathscr{H}(s))}=v_{\mathscr{A}}(s)
$$

for all $s \in \mathscr{V}$,

$\overline{\mu_{\mathscr{B}}(\mathscr{H}(s) \mathscr{H}(t))}=\mu_{\mathscr{B}}(s t), \quad \overline{v_{\mathscr{B}}(\mathscr{H}(s) \mathscr{H}(t))}=v_{\mathscr{B}}(s t)$ for all $s t \in E$.

Using the definition of complement of $G$, we have

$$
\begin{aligned}
\overline{\mu_{\mathscr{B}}(\mathscr{H}(s) \mathscr{H}(t))} & \frac{}{\mu_{\mathscr{A}}(\mathscr{H}(s)) \mu_{\mathscr{A}}(\mathscr{H}(t))} \\
= & \frac{\overline{\mu_{\mathscr{A}}(\mathscr{H}(s))}+\overline{\mu_{\mathscr{A}}(\mathscr{H}(t))}-\overline{\mu_{\mathscr{A}}(\mathscr{H}(s)) \mu_{\mathscr{A}}(\mathscr{H}(t))}}{} \\
& -\mu_{\mathscr{B}}(\mathscr{H}(s) \mathscr{H}(t))
\end{aligned}
$$




$$
\begin{aligned}
& \mu_{\mathscr{B}}(s t) \\
& =\frac{\mu_{\mathscr{A}}(s) \mu_{\mathscr{A}}(t)}{\mu_{\mathscr{A}}(s)+\mu_{\mathscr{A}}(t)-\mu_{\mathscr{A}}(s) \mu_{\mathscr{A}}(t)} \\
& \sum_{s \neq t} \mu_{\mathscr{B}}(s t) \\
& =\sum_{s \neq t} \frac{\mu_{\mathscr{A}}(s) \mu_{\mathscr{A}}(t)}{\mu_{\mathscr{A}}(s)+\mu_{\mathscr{A}}(t)-\mu_{\mathscr{A}}(s) \mu_{\mathscr{A}}(t)} \\
& \quad-\sum_{s \neq t} \mu_{\mathscr{B}}(\mathscr{H}(s) \mathscr{H}(t)) \\
& \sum_{s \neq t} \mu_{\mathscr{B}}(s t)+\sum_{s \neq t} \mu_{\mathscr{B}}(\mathscr{H}(s) \mathscr{H}(t)) \\
& =\sum_{s \neq t} \frac{\mu_{\mathscr{A}}(s) \mu_{\mathscr{A}}(t)}{\mu_{\mathscr{A}}(s)+\mu_{\mathscr{A}}(t)-\mu_{\mathscr{A}}(s) \mu_{\mathscr{A}}(t)} \\
& 2 \sum_{s \neq t} \mu_{\mathscr{B}}(s t) \\
& =\sum_{s \neq t} \frac{\mu_{\mathscr{A}}(s) \mu_{\mathscr{A}}(t)}{\mu_{\mathscr{A}}(s)+\mu_{\mathscr{A}}(t)-\mu_{\mathscr{A}}(s) \mu_{\mathscr{A}}(t)} \\
& \sum_{s \neq t} \mu_{\mathscr{B}}(s t) \\
& =\frac{1}{2} \sum_{s \neq t} \frac{\mu_{\mathscr{A}}(s)+\mu_{\mathscr{A}}(t)-\mu_{\mathscr{A}}(s) \mu_{\mathscr{A}}(t)}{\mu_{\mathscr{A}}(s) \mu_{\mathscr{A}}(t)}
\end{aligned}
$$

Likewise, for non-membership grade, we have

$$
\begin{aligned}
& \overline{v_{\mathscr{B}}(\mathscr{H}(s) \mathscr{H}(t))} \\
& -v_{\mathscr{B}}(\mathscr{H}(s) \mathscr{H}(t)) \\
& v_{\mathscr{B}}(s t) \\
& \begin{aligned}
= & \frac{v_{\mathscr{A}}(s)+v_{\mathscr{A}}(t)-2 v_{\mathscr{A}}(s) v_{\mathscr{A}}(t)}{1-v_{\mathscr{A}}(s) v_{\mathscr{A}}(t)} \\
& -v_{\mathscr{B}}(\mathscr{H}(s) \mathscr{H}(t))
\end{aligned} \\
& \sum_{s \neq t} v_{\mathscr{B}}(s t) \\
& =\sum_{s \neq t} \frac{v_{\mathscr{A}}(s)+v_{\mathscr{A}}(t)-2 v_{\mathscr{A}}(s) v_{\mathscr{A}}(t)}{1-v_{\mathscr{A}}(s) v_{\mathscr{A}}(t)} \\
& -\sum_{s \neq t} v_{\mathscr{B}}(\mathscr{H}(s) \mathscr{H}(t)) \\
& \sum_{s \neq t} v_{\mathscr{B}}(s t)+\sum_{s \neq t} v_{\mathscr{B}}(\mathscr{H}(s) \mathscr{H}(t)) \\
& =\sum_{s \neq t} \frac{v_{\mathscr{A}}(s)+v_{\mathscr{A}}(t)-2 v_{\mathscr{A}}(s) v_{\mathscr{A}}(t)}{1-v_{\mathscr{A}}(s) v_{\mathscr{A}}(t)}
\end{aligned}
$$$$
=\frac{\overline{v_{\mathscr{A}}(\mathscr{H}(s))}+\overline{v_{\mathscr{A}}(\mathscr{H}(t))}-2 \overline{v_{\mathscr{A}}(\mathscr{H}(s)) v_{\mathscr{A}}(\mathscr{H}(t))}}{1-\overline{v_{\mathscr{A}}(\mathscr{H}(s)) v_{\mathscr{A}}(\mathscr{H}(t))}}
$$

$$
\begin{aligned}
& 2 \sum_{s \neq t} v_{\mathscr{B}}(s t) \\
& \quad=\sum_{s \neq t} \frac{v_{\mathscr{A}}(s)+v_{\mathscr{A}}(t)-2 v_{\mathscr{A}}(s) v_{\mathscr{A}}(t)}{1-v_{\mathscr{A}}(s) v_{\mathscr{A}}(t)} \\
& \sum_{s \neq t} v_{\mathscr{B}}(s t) \\
& \quad=\frac{1}{2} \sum_{s \neq t} \frac{v_{\mathscr{A}}(s)+v_{\mathscr{A}}(t)-2 v_{\mathscr{A}}(s) v_{\mathscr{A}}(t)}{1-v_{\mathscr{A}}(s) v_{\mathscr{A}}(t)} .
\end{aligned}
$$

This completes the proof.

Proposition 2 Let $G=(\mathscr{A}, \mathscr{B})$ be the PDFG on underlying graph $G^{*}=(\mathscr{V}, E)$. If

$$
\begin{aligned}
\mu_{\mathscr{B}}(s t) & =\frac{1}{2}\left(\frac{\mu_{\mathscr{A}}(s) \mu_{\mathscr{A}}(t)}{\mu_{\mathscr{A}}(s)+\mu_{\mathscr{A}}(t)-\mu_{\mathscr{A}}(s) \mu_{\mathscr{A}}(t)}\right), \\
v_{\mathscr{B}}(s t) & =\frac{1}{2}\left(\frac{v_{\mathscr{A}}(s)+v_{\mathscr{A}}(t)-2 v_{\mathscr{A}}(s) \nu_{\mathscr{A}}(t)}{1-v_{\mathscr{A}}(s) v_{\mathscr{A}}(t)}\right)
\end{aligned}
$$

for all $s, t \in \mathscr{V}$, then $G$ is self-complementary.

Proof Assume that $G$ is the PDFG that satisfies

$$
\begin{aligned}
\mu_{\mathscr{B}}(s t) & =\frac{1}{2}\left(\frac{\mu_{\mathscr{A}}(s) \mu_{\mathscr{A}}(t)}{\mu_{\mathscr{A}}(s)+\mu_{\mathscr{A}}(t)-\mu_{\mathscr{A}}(s) \mu_{\mathscr{A}}(t)}\right), \\
v_{\mathscr{B}}(s t) & =\frac{1}{2}\left(\frac{v_{\mathscr{A}}(s)+v_{\mathscr{A}}(t)-2 v_{\mathscr{A}}(s) \nu_{\mathscr{A}}(t)}{1-v_{\mathscr{A}}(s) v_{\mathscr{A}}(t)}\right)
\end{aligned}
$$

for all $s, t \in \mathscr{V}$, then the identity mapping I $: \mathscr{V} \longrightarrow \mathscr{V}$ is an isomorphism from $G$ to $\bar{G}$ that satisfies the following conditions:

$$
\begin{aligned}
\mu_{\mathscr{A}}(s) & =\overline{\mu_{\mathscr{A}}(\mathrm{I}(s))} \text { and } v_{\mathscr{A}}(s) \\
& =\overline{v_{\mathscr{A}}(\mathrm{I}(s))} \text { for all } s \in \mathscr{V} .
\end{aligned}
$$

Since the membership grade of an edge $s t$ is given by

$$
\begin{aligned}
& \mu_{\mathscr{B}}(s t) \\
& \quad=\frac{1}{2}\left(\frac{\mu_{\mathscr{A}}(s) \mu_{\mathscr{A}}(t)}{\mu_{\mathscr{A}}(s)+\mu_{\mathscr{A}}(t)-\mu_{\mathscr{A}}(s) \mu_{\mathscr{A}}(t)}\right) \text { for all } s, t \in \mathscr{V},
\end{aligned}
$$

we have

$$
\begin{aligned}
\overline{\mu_{\mathscr{B}}(\mathrm{I}(s) \mathrm{I}(t))} & =\overline{\mu_{\mathscr{B}}(s t)} \\
= & \frac{\mu_{\mathscr{A}}(s) \mu_{\mathscr{A}}(t)}{\mu_{\mathscr{A}}(s)+\mu_{\mathscr{A}}(t)-\mu_{\mathscr{A}}(s) \mu_{\mathscr{A}}(t)} \\
& -\mu_{\mathscr{B}}(s t) \\
= & \frac{\mu_{\mathscr{A}}(s) \mu_{\mathscr{A}}(t)}{\mu_{\mathscr{A}}(s)+\mu_{\mathscr{A}}(t)-\mu_{\mathscr{A}}(s) \mu_{\mathscr{A}}(t)} \\
& -\frac{1}{2}\left(\frac{\mu_{\mathscr{A}}(s) \mu_{\mathscr{A}}(t)}{\mu_{\mathscr{A}}(s)+\mu_{\mathscr{A}}(t)-\mu_{\mathscr{A}}(s) \mu_{\mathscr{A}}(t)}\right)
\end{aligned}
$$




$$
\begin{aligned}
& =\frac{1}{2}\left(\frac{\mu_{\mathscr{A}}(s) \mu_{\mathscr{A}}(t)}{\mu_{\mathscr{A}}(s)+\mu_{\mathscr{A}}(t)-\mu_{\mathscr{A}}(s) \mu_{\mathscr{A}}(t)}\right) \\
& =\mu_{\mathscr{B}}(s t) .
\end{aligned}
$$

Likewise, the non-membership grade of an edge $s t$ is given by

$$
\begin{aligned}
& v_{\mathscr{B}}(s t) \\
& \quad=\frac{1}{2}\left(\frac{v_{\mathscr{A}}(s)+v_{\mathscr{A}}(t)-2 v_{\mathscr{A}}(s) v_{\mathscr{A}}(t)}{1-v_{\mathscr{A}}(s) v_{\mathscr{A}}(t)}\right) \text { for all } s, t \in \mathscr{V} ;
\end{aligned}
$$

so we have

$$
\begin{aligned}
\overline{v_{\mathscr{B}}(\mathrm{I}(s) \mathrm{I}(t))}= & \overline{v_{\mathscr{B}}(s t)} \\
= & \frac{v_{\mathscr{A}}(s)+v_{\mathscr{A}}(t)-2 v_{\mathscr{A}}(s) v_{\mathscr{A}}(t)}{1-v_{\mathscr{A}}(s) v_{\mathscr{A}}(t)}-v_{\mathscr{B}}(s t) \\
= & \frac{v_{\mathscr{A}}(s)+v_{\mathscr{A}}(t)-2 v_{\mathscr{A}}(s) v_{\mathscr{A}}(t)}{1-v_{\mathscr{A}}(s) v_{\mathscr{A}}(t)} \\
& -\frac{1}{2}\left(\frac{v_{\mathscr{A}}(s)+v_{\mathscr{A}}(t)-2 v_{\mathscr{A}}(s) v_{\mathscr{A}}(t)}{1-v_{\mathscr{A}}(s) v_{\mathscr{A}}(t)}\right) \\
= & \frac{1}{2}\left(\frac{v_{\mathscr{A}}(s)+v_{\mathscr{A}}(t)-2 v_{\mathscr{A}}(s) v_{\mathscr{A}}(t)}{1-v_{\mathscr{A}}(s) v_{\mathscr{A}}(t)}\right) \\
& =v_{\mathscr{B}}(s t) .
\end{aligned}
$$

Since the conditions of isomorphism $\overline{\mu_{\mathscr{B}}(\mathrm{I}(s) \mathrm{I}(t))}=\mu_{\mathscr{B}}(s t)$ and $\overline{v_{\mathscr{B}}(\mathrm{I}(s) \mathrm{I}(t))}=v_{\mathscr{B}}(s t)$ are satisfied by $\mathrm{I}, G=(\mathscr{A}, \mathscr{B})$ is self-complementary.

Proposition 3 If $G_{1}=\left(\mathscr{A}_{1}, \mathscr{B}_{1}\right)$ and $G_{2}=\left(\mathscr{A}_{2}, \mathscr{B}_{2}\right)$ are two isomorphic PDFGs, then the complement of $G_{1}$ and $G_{2}$ are also isomorphic to each other and conversely.

Proof Assume that $G_{1}$ and $G_{2}$ are two isomorphic PDFGs. Then by definition of isomorphism, there occurs a bijective mapping $\mathscr{H}: \mathscr{V}_{1} \longrightarrow \mathscr{V}_{2}$ that satisfies

$$
\begin{aligned}
\mu_{\mathscr{A}_{1}}(s) & =\mu_{\mathscr{A}_{2}}(\mathscr{H}(s)), v_{\mathscr{A}_{1}}(s) \\
& =v_{\mathscr{A}_{2}}(\mathscr{H}(s)) \text { for all } s \in \mathscr{V}_{1}, \\
\mu_{\mathscr{B}_{1}}(s t) & =\mu_{\mathscr{B}_{2}}(\mathscr{H}(s) \mathscr{H}(t)), v_{\mathscr{B}_{1}}(s t) \\
& =v_{\mathscr{B}_{2}}(\mathscr{H}(s) \mathscr{H}(t)) \text { for all } s t \in E_{1} .
\end{aligned}
$$

By using the definition of complement of PDFG, the membership grade of an edge $s t$ is

$$
\begin{aligned}
& \overline{\mu_{\mathscr{B}_{1}}(s t)} \\
&=\frac{\mu_{\mathscr{A}_{1}}(s) \mu_{\mathscr{A}_{1}}(t)}{\mu_{\mathscr{A}_{1}}(s)+\mu_{\mathscr{A}_{1}}(t)-\mu_{\mathscr{A}_{1}}(s) \mu_{\mathscr{A}_{1}}(t)}-\mu_{\mathscr{B}_{1}}(s t) \\
&=\frac{\mu_{\mathscr{A}_{2}}(\mathscr{H}(s)) \mu_{\mathscr{A}_{2}}(\mathscr{H}(t))}{\mu_{\mathscr{A}_{2}}(\mathscr{H}(s))+\mu_{\mathscr{A}_{2}}(\mathscr{H}(t))-\mu_{\mathscr{A}_{2}}(\mathscr{H}(s)) \mu_{\mathscr{A}_{2}}(\mathscr{H}(t))} \\
&= \frac{-\mu_{\mathscr{B}_{2}}(\mathscr{H}(s) \mathscr{H}(t))}{\mu_{\mathscr{B}_{2}}(\mathscr{H}(s) \mathscr{H}(t)) .}
\end{aligned}
$$

Also, the non-membership grade of an edge $s t$ is

$$
\begin{aligned}
\overline{v_{\mathscr{B}_{1}}(s t)} & \\
= & \frac{v_{\mathscr{A}_{1}}(s)+v_{\mathscr{A}_{1}}(t)-2 v_{\mathscr{A}_{1}}(s) v_{\mathscr{A}_{1}}(t)}{1-v_{\mathscr{A}_{1}}(s) v_{\mathscr{A}_{1}}(t)} \\
& -v_{\mathscr{B}_{1}}(s t) \\
= & \frac{v_{\mathscr{L}_{2}}(\mathscr{H}(s))+v_{\mathscr{A}_{2}}(\mathscr{H}(t))-2 v_{\mathscr{A}_{2}}(\mathscr{H}(s)) v_{\mathscr{A}_{2}}(\mathscr{H}(t))}{1-v_{\mathscr{A}_{2}}(\mathscr{H}(s)) v_{\mathscr{A}_{2}}(\mathscr{H}(t))} \\
= & \frac{-v_{\mathscr{B}_{2}}(\mathscr{H}(s) \mathscr{H}(t))}{v_{\mathscr{B}_{2}}(\mathscr{H}(s) \mathscr{H}(t)) .}
\end{aligned}
$$

Hence the complement of $G_{1}$ is isomorphic to the complement of $G_{2}$. Likewise, the converse part can be proved.

Proposition 4 If $G_{1}=\left(\mathscr{A}_{1}, \mathscr{B}_{1}\right)$ and $G_{2}=\left(\mathscr{A}_{2}, \mathscr{B}_{2}\right)$ are two weak isomorphic PDFGs, then the complement of $G_{1}$ and $G_{2}$ are also weak isomorphic.

Proof Assume that $G_{1}$ and $G_{2}$ are two weak isomorphic PDFGs. Then by definition of weak isomorphism, there occurs a bijective mapping $\mathscr{H}: \mathscr{V}_{1} \longrightarrow \mathscr{V}_{2}$ that satisfies

$$
\begin{aligned}
\mu_{\mathscr{A}_{1}}(s) & =\mu_{\mathscr{S}_{2}}(\mathscr{H}(s)), v_{\mathscr{A}_{1}}(s) \\
& =v_{\mathscr{A}_{2}}(\mathscr{H}(s)) \text { for all } s \in \mathscr{V}_{1}, \\
\mu_{\mathscr{B}_{1}}(s t) & \leq \mu_{\mathscr{B}_{2}}(\mathscr{H}(s) \mathscr{H}(t)), v_{\mathscr{B}_{1}}(s t) \\
& \leq v_{\mathscr{B}_{2}}(\mathscr{H}(s) \mathscr{H}(t)) \text { for all } s t \in E_{1}
\end{aligned}
$$

Consider the membership condition of an edge; then we have

$$
\begin{aligned}
& \mu_{\mathscr{B}_{1}}(s t) \leq \mu_{\mathscr{B}_{2}}(\mathscr{H}(s) \mathscr{H}(t)) \\
&-\mu_{\mathscr{B}_{1}}(s t) \geq-\mu_{\mathscr{B}_{2}}(\mathscr{H}(s) \mathscr{H}(t)) \\
& \mathcal{T}\left(\mu_{\mathscr{A}_{1}}(s), \mu_{\mathscr{A}_{1}}(t)\right)-\mu_{\mathscr{B}_{1}}(s t) \geq \mathcal{T}\left(\mu_{\mathscr{A}_{1}}(s), \mu_{\mathscr{A}_{1}}(t)\right) \\
&-\mu_{\mathscr{B}_{2}}(\mathscr{H}(s) \mathscr{H}(t)) \\
& \mathcal{T}\left(\mu_{\mathscr{A}_{1}}(s), \mu_{\mathscr{A}_{1}}(t)\right)-\mu_{\mathscr{B}_{1}}(s t) \geq \mathcal{T}\left(\mu_{\mathscr{A}_{2}}(\mathscr{H}(s)), \mu_{\mathscr{A}_{2}}(\mathscr{H}(t))\right) \\
&-\mu_{\mathscr{B}_{2}}(\mathscr{H}(s) \mathscr{H}(t)) \\
& \mu_{\mathscr{B}_{1}}(s t) \geq \\
& \mu_{\mathscr{B}_{2}}(\mathscr{H}(s) \mathscr{H}(t)) .
\end{aligned}
$$

Now take the non-membership condition of an edge; then we have

$$
\begin{aligned}
v_{\mathscr{B}_{1}}(s t) & \leq v_{\mathscr{B}_{2}}(\mathscr{H}(s) \mathscr{H}(t)) \\
-v_{\mathscr{B}_{1}}(s t) & \geq-v_{\mathscr{B}_{2}}(\mathscr{H}(s) \mathscr{H}(t)) \\
\mathscr{S}\left(v_{\mathscr{A}_{1}}(s), v_{\mathscr{A}_{1}}(t)\right)-v_{\mathscr{B}_{1}}(s t) \geq & \mathscr{S}\left(v_{\mathscr{A}_{1}}(s), v_{\mathscr{A}_{1}}(t)\right) \\
& -v_{\mathscr{B}_{2}}(\mathscr{H}(s) \mathscr{H}(t)) \\
\mathscr{S}\left(v_{\mathscr{A}_{1}}(s), v_{\mathscr{A}_{1}}(t)\right)-v_{\mathscr{B}_{1}}(s t) & \geq \mathscr{S}\left(v_{\mathscr{A}_{2}}(\mathscr{H}(s)), v_{\mathscr{A}_{2}}(\mathscr{H}(t))\right) \\
& -v_{\mathscr{B}_{2}}(\mathscr{H}(s) \mathscr{H}(t)) \\
v_{\mathscr{B}_{1}}(s t) & \geq v_{\mathscr{B}_{2}}(\mathscr{H}(s) \mathscr{H}(t))
\end{aligned}
$$

Hence we conclude that $\overline{G_{1}}$ and $\overline{G_{2}}$ are weak isomorphic. 


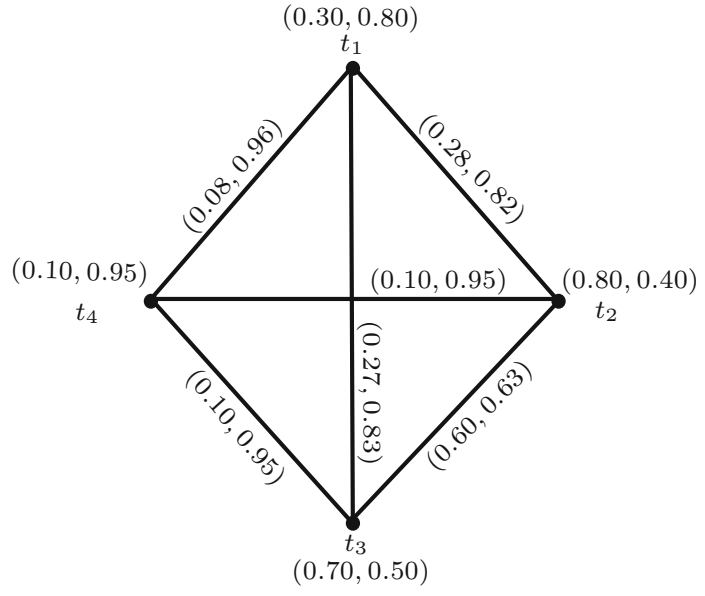

Fig. 5 Complete Pythagorean Dombi fuzzy graph

We state the following proposition without proof:

Proposition 5 If $G_{1}=\left(\mathscr{A}_{1}, \mathscr{B}_{1}\right)$ and $G_{2}=\left(\mathscr{A}_{2}, \mathscr{B}_{2}\right)$ are two co-weak isomorphic PDFGs, then the complement of $G_{1}$ and $G_{2}$ are homomorphic.

Definition 22 A PDFG is said to be complete if

$$
\begin{aligned}
& \mu_{\mathscr{B}}(s t)=\frac{\mu_{\mathscr{A}}(s) \mu_{\mathscr{A}}(t)}{\mu_{\mathscr{A}}(s)+\mu_{\mathscr{A}}(t)-\mu_{\mathscr{A}}(s) \mu_{\mathscr{A}}(t)}, \\
& v_{\mathscr{B}}(s t) \\
& \quad=\frac{v_{\mathscr{A}}(s)+v_{\mathscr{A}}(t)-2 v_{\mathscr{A}}(s) v_{\mathscr{A}}(t)}{1-v_{\mathscr{A}}(s) v_{\mathscr{A}}(t)} \text { for all } s, t \in \mathscr{V} .
\end{aligned}
$$

Example 5 Consider a graph $G^{*}=(\mathscr{V}, E)$, where $\mathscr{V}=$ $\left\{t_{1}, t_{2}, t_{3}, t_{4}\right\}$ and $E=\left\{t_{1} t_{2}, t_{1} t_{3}, t_{1} t_{4}, t_{2} t_{3}, t_{2} t_{4}, t_{3} t_{4}\right\}$. Let $\mathscr{A}$ and $\mathscr{B}$ be Pythagorean Dombi fuzzy vertex set and Pythagorean Dombi fuzzy edge set defined on $\mathscr{V}$ and $E$, respectively.

$$
\begin{aligned}
\mathscr{A}= & \left\langle\left(\frac{t_{1}}{0.30}, \frac{t_{2}}{0.80}, \frac{t_{3}}{0.70}, \frac{t_{4}}{0.10}\right),\right. \\
& \left.\left(\frac{t_{1}}{0.80}, \frac{t_{2}}{0.40}, \frac{t_{3}}{0.50}, \frac{t_{4}}{0.95}\right)\right\rangle \text { and } \\
\mathscr{B}= & \left\langle\left(\frac{t_{1} t_{2}}{0.28}, \frac{t_{1} t_{3}}{0.27}, \frac{t_{1} t_{4}}{0.08}, \frac{t_{2} t_{3}}{0.60}, \frac{t_{2} t_{4}}{0.10}, \frac{t_{3} t_{4}}{0.10}\right),\right. \\
& \left.\left(\frac{t_{1} t_{2}}{0.82}, \frac{t_{1} t_{3}}{0.83}, \frac{t_{1} t_{4}}{0.96}, \frac{t_{2} t_{3}}{0.63}, \frac{t_{2} t_{4}}{0.95}, \frac{t_{3} t_{4}}{0.95}\right)\right\rangle .
\end{aligned}
$$

By routine computations, one can see from Fig. 5 that $G=(\mathscr{A}, \mathscr{B})$ is a complete PDFG.

Definition 23 A PDFG is said to be strong if

$$
\mu_{\mathscr{B}}(s t)=\frac{\mu_{\mathscr{A}}(s) \mu_{\mathscr{A}}(t)}{\mu_{\mathscr{A}}(s)+\mu_{\mathscr{A}}(t)-\mu_{\mathscr{A}}(s) \mu_{\mathscr{A}}(t)},
$$

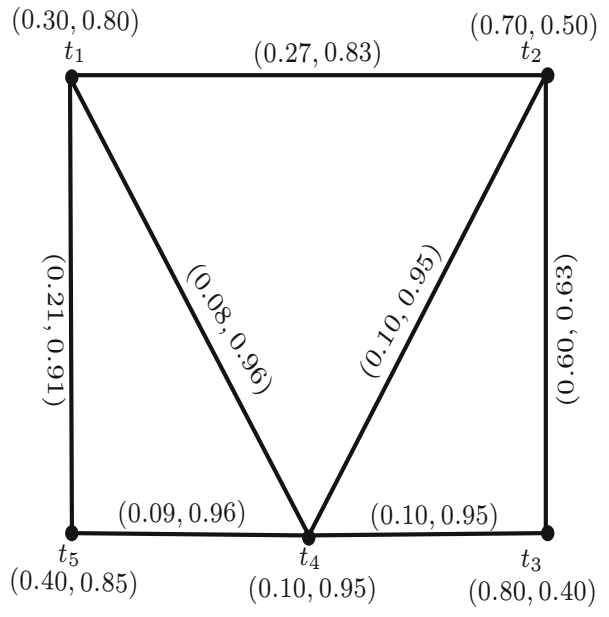

Fig. 6 Strong Pythagorean Dombi fuzzy graph

$$
\begin{aligned}
& v_{\mathscr{B}}(s t) \\
& \quad=\frac{v_{\mathscr{A}}(s)+v_{\mathscr{A}}(t)-2 v_{\mathscr{A}}(s) v_{\mathscr{A}}(t)}{1-v_{\mathscr{A}}(s) v_{\mathscr{A}}(t)} \text { for all } s t \in E .
\end{aligned}
$$

Example 6 Consider a graph $G^{*}=(\mathscr{V}, E)$, where $\mathscr{V}=$ $\left\{t_{1}, t_{2}, t_{3}, t_{4}, t_{5}\right\}$ and $E=\left\{t_{1} t_{2}, t_{1} t_{4}, t_{1} t_{5}, t_{2} t_{3}, t_{2} t_{4}, t_{3} t_{4}, t_{4} t_{5}\right\}$. Let $\mathscr{A}$ and $\mathscr{B}$ be Pythagorean Dombi fuzzy vertex set and Pythagorean Dombi fuzzy edge set defined on $\mathscr{V}$ and $E$, respectively.

$$
\begin{aligned}
\mathscr{A}= & \left\langle\left(\frac{t_{1}}{0.30}, \frac{t_{2}}{0.70}, \frac{t_{3}}{0.80}, \frac{t_{4}}{0.10}, \frac{t_{5}}{0.40}\right),\right. \\
& \left.\left(\frac{t_{1}}{0.80}, \frac{t_{2}}{0.50}, \frac{t_{3}}{0.40}, \frac{t_{4}}{0.95}, \frac{t_{5}}{0.85}\right)\right\rangle \text { and } \\
\mathscr{B}= & \left\langle\left(\frac{t_{1} t_{2}}{0.27}, \frac{t_{1} t_{4}}{0.08}, \frac{t_{1} t_{5}}{0.21}, \frac{t_{2} t_{3}}{0.60}, \frac{t_{2} t_{4}}{0.10}, \frac{t_{3} t_{4}}{0.10}, \frac{t_{4} t_{5}}{0.09}\right),\right. \\
& \left.\left(\frac{t_{1} t_{2}}{0.83}, \frac{t_{1} t_{4}}{0.96}, \frac{t_{1} t_{5}}{0.91}, \frac{t_{2} t_{3}}{0.63}, \frac{t_{2} t_{4}}{0.95}, \frac{t_{3} t_{4}}{0.95}, \frac{t_{4} t_{5}}{0.96}\right)\right\rangle .
\end{aligned}
$$

By routine computations, one can see from Fig. 6 that $G=(\mathscr{A}, \mathscr{B})$ is a strong PDFG.

Remark 2 Every complete PDFG is strong; one can easily see from Example 5.

Definition 24 The complement of a strong PDFG $G=$ $(\mathscr{A}, \mathscr{B})$ on an underlying graph $G^{*}=(\mathscr{V}, E)$ is a PDFG $\bar{G}=(\overline{\mathscr{A}}, \overline{\mathscr{B}})$ defined by

$$
\begin{aligned}
& \text { 1. } \overline{\mu_{\mathscr{A}}(s)}=\mu_{\mathscr{A}}(s) \text { and } \overline{v_{\mathscr{A}}(s)}=v_{\mathscr{A}}(s) \text { for all } s \in \mathscr{V} . \\
& \text { 2. } \overline{\mu_{\mathscr{B}}(s t)}= \begin{cases}\frac{\mu_{\mathscr{A}}(s) \mu_{\mathscr{A}}(t)}{\mu_{\mathscr{A}}(s)+\mu_{\mathscr{A}}(t)-\mu_{\mathscr{A}}(s) \mu_{\mathscr{A}}(t)}, & \text { if } \mu_{\mathscr{B}}(s t)=0, \\
0, & \text { if } 0<\mu_{\mathscr{B}}(s t) \leq 1 .\end{cases} \\
& \overline{\nu_{\mathscr{B}}(s t)}= \begin{cases}\frac{v_{\mathscr{A}}(s)+\nu_{\mathscr{A}}(t)-2 v_{\mathscr{A}}(s) \nu_{\mathscr{A}}(t)}{1-v_{\mathscr{A}}(s) \nu_{\mathscr{A}}(t)}, & \text { if } \nu_{\mathscr{B}}(s t)=0, \\
0, & \text { if } 0<v_{\mathscr{B}}(s t) \leq 1 .\end{cases}
\end{aligned}
$$




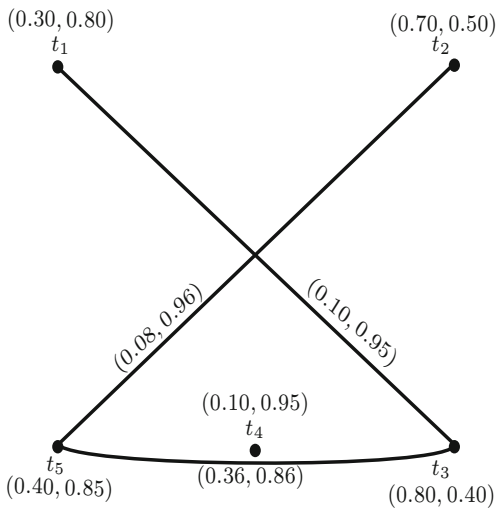

Fig. 7 Complement of a strong Pythagorean Dombi fuzzy graph

Example 7 Consider a strong PDFG $G=(\mathscr{A}, \mathscr{B})$ as displayed in Fig. 6. Then by using the Definition 24, one can obtain complement of a strong PDFG as presented in Fig. 7.

By routine computations, one can see from Fig. 7 that $\bar{G}$ is a PDFG.

\section{Regularity of Pythagorean Dombi fuzzy graphs}

In this section, we introduce the concept of regularity of Pythagorean Dombi fuzzy graphs that can be helpful in real scientific applications.

Definition 25 A PDFG $G=(\mathscr{A}, \mathscr{B})$ on crisp graph $G^{*}=$ $(\mathscr{V}, E)$ is said to be regular of degree $\left(\mathscr{R}_{1}, \mathscr{R}_{2}\right)$ or $\left(\mathscr{R}_{1}, \mathscr{R}_{2}\right)$ regular. If

$$
\begin{aligned}
(\mathscr{D})_{\mu}(s) & =\sum_{s, t \neq s \in \mathscr{V}} \mu_{\mathscr{B}}(s t) \\
& =\sum_{s, t \neq s \in \mathscr{V}} \frac{\mu_{\mathscr{A}}(s) \mu_{\mathscr{A}}(t)}{\mu_{\mathscr{A}}(s)+\mu_{\mathscr{A}}(t)-\mu_{\mathscr{A}}(s) \mu_{\mathscr{A}}(t)}=\mathscr{R}_{1}, \\
(\mathscr{D})_{\nu}(s) & =\sum_{s, t \neq s \in \mathscr{V}} v_{\mathscr{B}}(s t) \\
& =\sum_{s, t \neq s \in \mathscr{V}} \frac{v_{\mathscr{A}}(s)+v_{\mathscr{A}}(t)-2 v_{\mathscr{A}}(s) v_{\mathscr{A}}(t)}{1-v_{\mathscr{A}}(s) v_{\mathscr{A}}(t)} \\
& =\mathscr{R}_{2} \text { for all } \mathrm{s} \in \mathscr{V} .
\end{aligned}
$$

Example 8 Consider a graph $G^{*}=(\mathscr{V}, E)$, where $\mathscr{V}=$ $\left\{t_{1}, t_{2}, t_{3}, t_{4}\right\}$ and $E=\left\{t_{1} t_{2}, t_{1} t_{4}, t_{2} t_{3}, t_{3} t_{4}\right\}$. Let $\mathscr{A}$ and $\mathscr{B}$ be Pythagorean Dombi fuzzy vertex set and Pythagorean Dombi fuzzy edge set defined on $\mathscr{V}$ and $E$, respectively.

$$
\mathscr{A}=\left\langle\left(\frac{t_{1}}{0.50}, \frac{t_{2}}{0.90}, \frac{t_{3}}{0.80}, \frac{t_{4}}{0.70}\right),\right.
$$

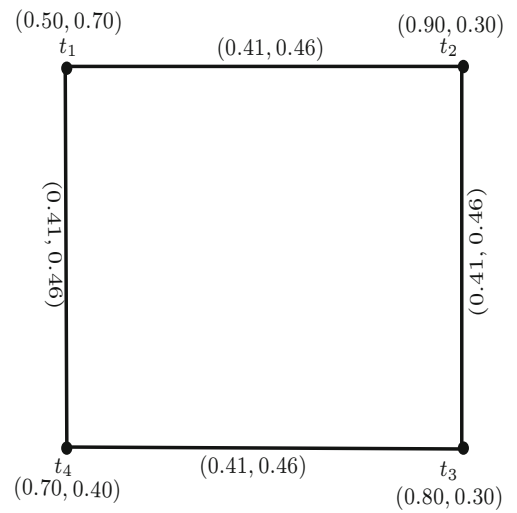

Fig. 8 (0.82, 0.92)-regular Pythagorean Dombi fuzzy graph

$$
\begin{aligned}
& \left.\left(\frac{t_{1}}{0.70}, \frac{t_{2}}{0.30}, \frac{t_{3}}{0.30}, \frac{t_{4}}{0.40}\right)\right\rangle \text { and } \\
\mathscr{B}= & \left\langle\left(\frac{t_{1} t_{2}}{0.41}, \frac{t_{1} t_{4}}{0.41}, \frac{t_{2} t_{3}}{0.41}, \frac{t_{3} t_{4}}{0.41}\right),\right. \\
& \left.\left(\frac{t_{1} t_{2}}{0.46}, \frac{t_{1} t_{4}}{0.46}, \frac{t_{2} t_{3}}{0.46}, \frac{t_{3} t_{4}}{0.46}\right)\right\rangle .
\end{aligned}
$$

Since $(\mathscr{D})_{G}\left(t_{1}\right)=(\mathscr{D})_{G}\left(t_{2}\right)=(\mathscr{D})_{G}\left(t_{3}\right)=(\mathscr{D})_{G}\left(t_{4}\right)=$ $(0.82,0.92), G=(\mathscr{A}, \mathscr{B})$, presented in Fig. 8, is $(0.82,0.92)$-regular PDFG.

Definition 26 A PDFG $G=(\mathscr{A}, \mathscr{B})$ on crisp graph $G^{*}=$ $(\mathscr{V}, E)$ is said to be totally regular of degree $\left(\mathscr{K}_{1}, \mathscr{K}_{2}\right)$ or $\left(\mathscr{K}_{1}, \mathscr{K}_{2}\right)$-totally regular. If

$$
\begin{aligned}
(\mathscr{T} \mathscr{D})_{\mu}(s)= & \sum_{s, t \neq s \in \mathscr{Y}} \mu_{\mathscr{B}}(s t)+\mu_{\mathscr{A}}(s) \\
= & \sum_{s, t \neq s \in \mathscr{V}} \frac{\mu_{\mathscr{A}}(s) \mu_{\mathscr{A}}(t)}{\mu_{\mathscr{A}}(s)+\mu_{\mathscr{A}}(t)-\mu_{\mathscr{A}}(s) \mu_{\mathscr{A}}(t)} \\
& +\mu_{\mathscr{A}}(s)=\mathscr{K}_{1}, \\
(\mathscr{T} \mathscr{D})_{v}(s)= & \sum_{s, t \neq s \in \mathscr{V}} v_{\mathscr{B}}(s t)+v_{\mathscr{A}}(s) \\
= & \sum_{s, t \neq s \in \mathscr{V}} \frac{v_{\mathscr{A}}(s)+v_{\mathscr{A}}(t)-2 v_{\mathscr{A}}(s) v_{\mathscr{A}}(t)}{1-v_{\mathscr{A}}(s) v_{\mathscr{A}}(t)}+v_{\mathscr{A}}(s) \\
= & \mathscr{K}_{2} \text { for all s } \in \mathscr{V} .
\end{aligned}
$$

Example 9 Consider a graph $G^{*}=(\mathscr{V}, E)$, where $\mathscr{V}=$ $\left\{t_{1}, t_{2}, t_{3}, t_{4}\right\}$ and $E=\left\{t_{1} t_{3}, t_{1} t_{4}, t_{2} t_{3}, t_{2} t_{4}\right\}$. Let $\mathscr{A}$ and $\mathscr{B}$ be Pythagorean Dombi fuzzy vertex set and Pythagorean Dombi fuzzy edge set defined on $\mathscr{V}$ and $E$, respectively.

$$
\begin{aligned}
\mathscr{A}= & \left\langle\left(\frac{t_{1}}{0.50}, \frac{t_{2}}{0.50}, \frac{t_{3}}{0.50}, \frac{t_{4}}{0.50}\right),\right. \\
& \left.\left(\frac{t_{1}}{0.60}, \frac{t_{2}}{0.60}, \frac{t_{3}}{0.60}, \frac{t_{4}}{0.60}\right)\right\rangle \text { and }
\end{aligned}
$$




$$
\begin{array}{r}
\mathscr{B}=\left\langle\left(\frac{t_{1} t_{3}}{0.25}, \frac{t_{1} t_{4}}{0.33}, \frac{t_{2} t_{3}}{0.33}, \frac{t_{2} t_{4}}{0.25}\right),\right. \\
\\
\left.\left(\frac{t_{1} t_{3}}{0.70}, \frac{t_{1} t_{4}}{0.74}, \frac{t_{2} t_{3}}{0.74}, \frac{t_{2} t_{4}}{0.70}\right)\right\rangle .
\end{array}
$$

Since $(\mathscr{T} \mathscr{D})_{G}\left(t_{1}\right)=(\mathscr{T} \mathscr{D})_{G}\left(t_{2}\right)=(\mathscr{T} \mathscr{D})_{G}\left(t_{3}\right)=$ $(\mathscr{T} \mathscr{D})_{G}\left(t_{4}\right)=(1.08,2.04), G=(\mathscr{A}, \mathscr{B})$, presented in Fig. 9 , is $(1.08,2.04)$-totally regular PDFG.

Theorem 2 Let $G=(\mathscr{A}, \mathscr{B})$ be a $\left(\mathscr{R}_{1}, \mathscr{R}_{2}\right)$-regular PDFG. Then the size $\mathscr{S}(G)=\left(\frac{n \mathscr{R}_{1}}{2}, \frac{n \mathscr{R}_{2}}{2}\right)$, where $|\mathscr{V}|=n$.

Proof Assume that $G$ is a PDFG with size given by

$\mathscr{S}(G)=\left(\sum_{s t \in E} \mu_{\mathscr{B}}(s t), \sum_{s t \in E} v_{\mathscr{B}}(s t)\right)$.

Since $G$ is $\left(\mathscr{R}_{1}, \mathscr{R}_{2}\right)$-regular, the degree of the vertex

$$
\begin{aligned}
& \left((\mathscr{D})_{\mu}(s),(\mathscr{D})_{\nu}(s)\right) \\
& =\left(\sum_{s, t \neq s \in \mathscr{V}} \mu_{\mathscr{B}}(s t), \sum_{s, t \neq s \in \mathscr{V}} v_{\mathscr{B}}(s t)\right) \\
& \left(\sum_{s \in \mathscr{V}}(\mathscr{D})_{\mu}(s), \sum_{s \in \mathscr{V}}(\mathscr{D})_{v}(s)\right) \\
& =\left(\sum_{s \in \mathscr{V}} \sum_{s, t \neq s \in \mathscr{V}} \frac{\mu_{\mathscr{A}}(s) \mu_{\mathscr{A}}(t)}{\mu_{\mathscr{A}}(s)+\mu_{\mathscr{A}}(t)-\mu_{\mathscr{A}}(s) \mu_{\mathscr{A}}(t)},\right. \\
& \left.\quad \sum_{s \in \mathscr{V}} \sum_{s, t \neq s \in \mathscr{V}} \frac{v_{\mathscr{A}}(s)+v_{\mathscr{A}}(t)-2 v_{\mathscr{A}}(s) v_{\mathscr{A}}(t)}{1-v_{\mathscr{A}}(s) v_{\mathscr{A}}(t)}\right)
\end{aligned}
$$

Since each edge is double times counted, one time for vertex $s$ and one time for $t$, we have

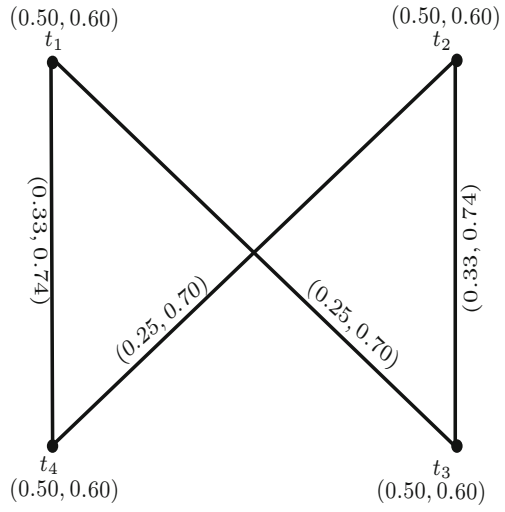

Fig. 9 (1.08, 2.04)-totally regular Pythagorean Dombi fuzzy graph

$$
\begin{gathered}
\left(\sum_{s \in \mathscr{V}}(\mathscr{D})_{\mu}(s), \sum_{s \in \mathscr{V}}(\mathscr{D})_{v}(s)\right) \\
=2\left(\sum_{s, t \neq s \in \mathscr{V}} \frac{\mu_{\mathscr{A}}(s) \mu_{\mathscr{A}}(t)}{\mu_{\mathscr{A}}(s)+\mu_{\mathscr{A}}(t)-\mu_{\mathscr{A}}(s) \mu_{\mathscr{A}}(t)},\right. \\
\left.\sum_{s, t \neq s \in \mathscr{V}} \frac{v_{\mathscr{A}}(s)+v_{\mathscr{A}}(t)-2 v_{\mathscr{A}}(s) v_{\mathscr{A}}(t)}{1-v_{\mathscr{A}}(s) v_{\mathscr{A}}(t)}\right) \\
\left(\sum_{s \in \mathscr{V}} \mathscr{R}_{1}, \sum_{s \in \mathscr{V}} \mathscr{R}_{2}\right)=2\left(\sum_{s t \in E} \mu_{\mathscr{B}}(s t), \sum_{s t \in E} v_{\mathscr{B}}(s t)\right) \\
\left(n \mathscr{R}_{1}, n \mathscr{R}_{2}\right)=2 \mathscr{S}(G) \\
\left(\frac{n \mathscr{R}_{1}}{2}, \frac{n \mathscr{R} 2}{2}\right)=\mathscr{S}(G) .
\end{gathered}
$$

This completes the proof.

Theorem 3 Let $G=(\mathscr{A}, \mathscr{B})$ be a $\left(\mathscr{K}_{1}, \mathscr{K}_{2}\right)$-totally regular PDFG. Then $2 \mathscr{S}(G)+\mathscr{O}(G)=\left(n \mathscr{K}_{1}, n \mathscr{K}_{2}\right)$, where $|\mathscr{V}|=$ $n$.

Proof Assume that $G$ is a PDFG; then the total degree is given by

$$
\begin{aligned}
& \left((\mathscr{T} \mathscr{D})_{\mu}(s),(\mathscr{T} \mathscr{D})_{v}(s)\right) \\
& \quad=\left(\sum_{s, t \neq s \in \mathscr{V}} \mu_{\mathscr{B}}(s t)+\mu_{\mathscr{A}}(s), \sum_{s, t \neq s \in \mathscr{V}} v_{\mathscr{B}}(s t)+v_{\mathscr{A}}(s)\right) .
\end{aligned}
$$

Since $G$ is $\left(\mathscr{K}_{1}, \mathscr{K}_{2}\right)$-totally regular,

$$
\begin{aligned}
\left(\mathscr{K}_{1}, \mathscr{K}_{2}\right) & \left(\sum_{s, t \neq s \in \mathscr{V}} \frac{\mu_{\mathscr{A}}(s) \mu_{\mathscr{A}}(t)}{\mu_{\mathscr{A}}(s)+\mu_{\mathscr{A}}(t)-\mu_{\mathscr{A}}(s) \mu_{\mathscr{A}}(t)}+\mu_{\mathscr{A}}(s),\right. \\
& \left.\sum_{s, t \neq s \in \mathscr{V}} \frac{v_{\mathscr{A}}(s)+v_{\mathscr{A}}(t)-2 v_{\mathscr{A}}(s) v_{\mathscr{A}}(t)}{1-v_{\mathscr{A}}(s) v_{\mathscr{A}}(t)}+v_{\mathscr{A}}(s)\right) \\
\left(\sum_{s \in \mathscr{V}} \mathscr{K}_{1}, \sum_{s \in \mathscr{V}} \mathscr{K}_{2}\right) & \left(\sum_{s \in \mathscr{V}} \sum_{s, t \neq s \in \mathscr{V}} \frac{\mu_{\mathscr{A}}(s) \mu_{\mathscr{A}}(t)}{\mu_{\mathscr{A}}(s)+\mu_{\mathscr{A}}(t)-\mu_{\mathscr{A}}(s) \mu_{\mathscr{A}}(t)}\right. \\
& +\sum_{s \in \mathscr{V}} \mu_{\mathscr{A}}(s), \\
& \sum_{s \in \mathscr{V}} \sum_{s, t \neq s \in \mathscr{V}} \frac{v_{\mathscr{A}}(s)+v_{\mathscr{A}}(t)-2 v_{\mathscr{A}}(s) v_{\mathscr{A}}(t)}{1-v_{\mathscr{A}}(s) v_{\mathscr{A}}(t)} \\
& \left.+\sum_{s \in \mathscr{V}} v_{\mathscr{A}}(s)\right) .
\end{aligned}
$$


Since each edge is double times counted, one time for vertex $s$ and one time for $t$, we have

$$
\begin{aligned}
& \left(n \mathscr{K}_{1}, n \mathscr{K}_{2}\right) \\
& =\left(2 \sum_{s, t \neq s \in \mathscr{V}} \frac{\mu_{\mathscr{A}}(s) \mu_{\mathscr{A}}(t)}{\mu_{\mathscr{A}}(s)+\mu_{\mathscr{A}}(t)-\mu_{\mathscr{A}}(s) \mu_{\mathscr{A}}(t)}\right. \\
& +\sum_{s \in \mathscr{V}} \mu_{\mathscr{A}}(s) \\
& 2 \sum_{s, t \neq s \in \mathscr{V}} \frac{v_{\mathscr{A}}(s)+v_{\mathscr{A}}(t)-2 v_{\mathscr{A}}(s) v_{\mathscr{A}}(t)}{1-v_{\mathscr{A}}(s) v_{\mathscr{A}}(t)} \\
& \left.+\sum_{s \in \mathscr{V}} v_{\mathscr{A}}(s)\right) \\
& \left(n \mathscr{K}_{1}, n \mathscr{K}_{2}\right) \\
& =2\left(\sum_{s, t \neq s \in \mathscr{V}} \frac{\mu_{\mathscr{A}}(s) \mu_{\mathscr{A}}(t)}{\mu_{\mathscr{A}}(s)+\mu_{\mathscr{A}}(t)-\mu_{\mathscr{A}}(s) \mu_{\mathscr{A}}(t)},\right. \\
& \left.\sum_{s, t \neq s \in \mathscr{V}} \frac{v_{\mathscr{A}}(s)+v_{\mathscr{A}}(t)-2 v_{\mathscr{A}}(s) v_{\mathscr{A}}(t)}{1-v_{\mathscr{A}}(s) v_{\mathscr{A}}(t)}\right) \\
& +\left(\sum_{s \in \mathscr{V}} \mu_{\mathscr{A}}(s), \sum_{s \in \mathscr{V}} v_{\mathscr{A}}(s)\right) \\
& \left(n \mathscr{K}_{1}, n \mathscr{K}_{2}\right) \\
& \begin{aligned}
= & 2\left(\sum_{s t \in E} \mu_{\mathscr{B}}(s t), \sum_{s t \in E} v_{\mathscr{B}}(s t)\right) \\
& +\left(\sum_{s \in \mathscr{V}} \mu_{\mathscr{A}}(s), \sum_{s \in \mathscr{V}} v_{\mathscr{A}}(s)\right)
\end{aligned} \\
& \left(n \mathscr{K}_{1}, n \mathscr{K}_{2}\right)=2 \mathscr{S}(G)+\mathscr{O}(G) .
\end{aligned}
$$

This completes the proof.

Corollary 1 Let $G=(\mathscr{A}, \mathscr{B})$ be a $\left(\mathscr{R}_{1}, \mathscr{R}_{2}\right)$-regular and $\left(\mathscr{K}_{1}, \mathscr{K}_{2}\right)$-totally regular PDFG. Then $\mathscr{O}(G)=n\left\{\left(\mathscr{K}_{1}, \mathscr{K}_{2}\right)\right.$ $\left.-\left(\mathscr{R}_{1}, \mathscr{R}_{2}\right)\right\}$ ( or $\left.n\left\{\left(\mathscr{K}_{1}-\mathscr{R}_{1}\right)+\left(\mathscr{K}_{2}-\mathscr{R}_{2}\right)\right\}\right)$.

Proof Assume that $G$ is a $\left(\mathscr{R}_{1}, \mathscr{R}_{2}\right)$-regular PDFG. Then the size of $G$ is

$\mathscr{S}(G)=\left(\frac{n \mathscr{R}_{1}}{2}, \frac{n \mathscr{R}_{2}}{2}\right)$ or $2 \mathscr{S}(G)=\left(n \mathscr{R}_{1}, n \mathscr{R}_{2}\right)$.

As $G$ is a $\left(\mathscr{K}_{1}, \mathscr{K}_{2}\right)$-totally regular PDFG, from Theorem 3, we must have

$$
\begin{aligned}
2 \mathscr{S}(G)+\mathscr{O}(G) & =\left(n \mathscr{K}_{1}, n \mathscr{K}_{2}\right) \\
\mathscr{O}(G) & =\left(n \mathscr{K}_{1}, n \mathscr{K}_{2}\right)-2 \mathscr{S}(G) \\
\mathscr{O}(G) & =\left(n \mathscr{K}_{1}, n \mathscr{K}_{2}\right)-\left(n \mathscr{R}_{1}, n \mathscr{R}_{2}\right) \\
\mathscr{O}(G) & =n\left\{\left(\mathscr{K}_{1}, \mathscr{K}_{2}\right)-\left(\mathscr{R}_{1}, \mathscr{R}_{2}\right)\right\} \\
\mathscr{O}(G) & =n\left\{\left(\mathscr{K}_{1}-\mathscr{R}_{1}\right)+\left(\mathscr{K}_{2}-\mathscr{R}_{2}\right)\right\} .
\end{aligned}
$$

This completes the proof.
Theorem 4 Consider $G_{1}=\left(\mathscr{A}_{1}, \mathscr{B}_{1}\right)$ is isomorphic to $G_{2}=$ $\left(\mathscr{A}_{2}, \mathscr{B}_{2}\right)$ :

1. If $G_{1}$ is regular $P D F G$, then $G_{2}$ is also regular $P D F G$.

2. If $G_{1}$ is totally regular $P D F G$, then $G_{2}$ is also totally regular $P D F G$.

Proof 1. Assume that $G_{1}$ is isomorphic to $G_{2}$ and $G_{1}$ is $\left(\mathscr{R}_{1}, \mathscr{R}_{2}\right)$-regular PDFG; then the degree of each vertex in $G_{1}$ is given by

$$
\begin{aligned}
(\mathscr{D})_{G_{1}}(s)= & \left((\mathscr{D})_{\mu_{1}}(s),(\mathscr{D})_{\nu_{1}}(s)\right) \\
= & \left(\sum_{s t \in E} \mu_{\mathscr{B}_{1}}(s t), \sum_{s t \in E} v_{\mathscr{B}_{1}}(s t)\right) \\
= & \left(\sum_{s, t \neq s \in \mathscr{V}} \frac{\mu_{\mathscr{A}_{1}}(s) \mu_{\mathscr{A}_{1}}(t)}{\mu_{\mathscr{A}_{1}}(s)+\mu_{\mathscr{A}_{1}}(t)-\mu_{\mathscr{A}_{1}}(s) \mu_{\mathscr{A}_{1}}(t)},\right. \\
& \left.\sum_{s, t \neq s \in \mathscr{V}} \frac{v_{\mathscr{A}_{1}}(s)+v_{\mathscr{A}_{1}}(t)-2 v_{\mathscr{A}_{1}}(s) v_{\mathscr{A}_{1}}(t)}{1-v_{\mathscr{A}_{1}}(s) v_{\mathscr{A}_{1}}(t)}\right) \\
= & \left(\mathscr{R}_{1}, \mathscr{R}_{2}\right) .
\end{aligned}
$$

Since $G_{1} \cong G_{2}$, we must have

$$
\begin{aligned}
& \left(\mathscr{R}_{1}, \mathscr{R}_{2}\right) \\
& =\left((\mathscr{D})_{\mu_{1}}(s),(\mathscr{D})_{\nu_{1}}(s)\right) \\
& =\left(\sum_{s t \in E} \mu_{\mathscr{B}}(s t), \sum_{s t \in E} v_{\mathscr{B}_{1}}(s t)\right) \\
& =\left(\sum_{s, t \neq s \in \mathscr{V}} \frac{\mu_{\mathscr{A}_{1}}(s) \mu_{\mathscr{A}_{1}}(t)}{\mu_{\mathscr{A}_{1}}(s)+\mu_{\mathscr{A}_{1}}(t)-\mu_{\mathscr{A}_{1}}(s) \mu_{\mathscr{A}_{1}}(t)},\right. \\
& \left.\sum_{s, t \neq s \in \mathscr{V}} \frac{v_{\mathscr{A}_{1}}(s)+v_{\mathscr{A}_{1}}(t)-2 v_{\mathscr{A}_{1}}(s) v_{\mathscr{A}_{1}}(t)}{1-v_{\mathscr{A}_{1}}(s) v_{\mathscr{A}_{1}}(t)}\right) \\
& =\left(\sum_{s, t \neq s \in \mathscr{V}} \frac{\mu_{\mathscr{A}_{2}}(\mathscr{H}(s)) \mu_{\mathscr{A}_{2}}(\mathscr{H}(t))}{\mu_{\mathscr{A}_{2}}(\mathscr{H}(s))+\mu_{\mathscr{A}_{2}}(\mathscr{H}(t))-\mu_{\mathscr{A}_{2}}(\mathscr{H}(s)) \mu_{\mathscr{A}_{2}}(\mathscr{H}(t))},\right. \\
& \left.\sum_{s, t \neq s \in \mathscr{V}} \frac{v_{\mathscr{A}_{2}}(\mathscr{H}(s))+v_{\mathscr{A}_{2}}(\mathscr{H}(t))-2 v_{\mathscr{A}_{2}}(\mathscr{H}(s)) v_{\mathscr{A}_{2}}(\mathscr{H}(t))}{1-v_{\mathscr{A}_{2}}(\mathscr{H}(s)) v_{\mathscr{A}_{2}}(\mathscr{H}(t))}\right) \\
& =\left(\sum_{s t \in E} \mu_{\mathscr{B}}{ }_{2}(\mathscr{H}(s) \mathscr{H}(t)), \sum_{s t \in E} v_{\mathscr{B}_{2}}(\mathscr{H}(s) \mathscr{H}(t))\right) \\
& =\left((\mathscr{D})_{\mu_{2}}(\mathscr{H}(s)),(\mathscr{D})_{\nu_{2}}(\mathscr{H}(s))\right) \text {. }
\end{aligned}
$$

Therefore, $G_{2}$ is a $\left(\mathscr{R}_{1}, \mathscr{R}_{2}\right)$-regular PDFG. em Assume that $G_{1}$ is isomorphic to $G_{2}$ and $G_{1}$ is $\left(\mathscr{K}_{1}, \mathscr{K}_{2}\right)$-totally regular PDFG, then the total degree of each vertex in $G_{1}$ is given by

$$
\begin{aligned}
(\mathscr{T} \mathscr{D})_{G_{1}}(s) & \\
& =\left((\mathscr{T} \mathscr{D})_{\mu_{1}}(s),(\mathscr{T} \mathscr{D})_{\nu_{1}}(s)\right) \\
& =\left(\sum_{s t \in E} \mu_{\mathscr{B}_{1}}(s t)+\mu_{\mathscr{A}_{1}}(s), \sum_{s t \in E} v_{\mathscr{B}_{1}}(s t)+v_{\mathscr{A}_{1}}(s)\right) \\
& =\left(\sum_{s, t \neq s \in \mathscr{V}} \frac{\mu_{\mathscr{A}_{1}}(s) \mu_{\mathscr{A}_{1}}(t)}{\mu_{\mathscr{A}_{1}}(s)+\mu_{\mathscr{A}_{1}}(t)-\mu_{\mathscr{A}_{1}}(s) \mu_{\mathscr{A}_{1}}(t)}+\mu_{\mathscr{A}_{1}}(s),\right.
\end{aligned}
$$




$$
\begin{aligned}
& \left.\sum_{s, t \neq s \in \mathscr{V}} \frac{v_{\mathscr{A}_{1}}(s)+v_{\mathscr{A}_{1}}(t)-2 v_{\mathscr{A}_{1}}(s) v_{\mathscr{A}_{1}}(t)}{1-v_{\mathscr{A}_{1}}(s) v_{\mathscr{A}_{1}}(t)}+v_{\mathscr{A}_{1}}(s)\right) \\
= & \left(\mathscr{K}_{1}, \mathscr{K}_{2}\right) .
\end{aligned}
$$

Since $G_{1} \cong G_{2}$, we must have

$$
\begin{aligned}
\left(\mathscr{K}_{1},\right. & \left.\mathscr{K}_{2}\right) \\
= & \left((\mathscr{T} \mathscr{D})_{\mu_{1}}(s),(\mathscr{T} \mathscr{D})_{v_{1}}(s)\right) \\
= & \left(\sum_{s t \in E} \mu_{\mathscr{B}_{1}}(s t)+\mu_{\mathscr{A}_{1}}(s), \sum_{s t \in E} v_{\mathscr{B}_{1}}(s t)+v_{\mathscr{A}_{1}}(s)\right) \\
= & \left(\sum_{s, t \neq s \in \mathscr{V}} \frac{\mu_{\mathscr{A}_{1}}(s) \mu_{\mathscr{A}_{1}}(t)}{\mu_{\mathscr{A}_{1}}(s)+\mu_{\mathscr{A}_{1}}(t)-\mu_{\mathscr{A}_{1}}(s) \mu_{\mathscr{A}_{1}}(t)}+\mu_{\mathscr{A}_{1}}(s),\right. \\
& \left.\sum_{s, t \neq s \in \mathscr{V}} \frac{v_{\mathscr{A}_{1}}(s)+v_{\mathscr{A}_{1}}(t)-2 v_{\mathscr{A}_{1}}(s) v_{\mathscr{A}_{1}}(t)}{1-v_{\mathscr{A}_{1}}(s) v_{\mathscr{A}_{1}}(t)}+v_{\mathscr{A}_{1}}(s)\right) \\
= & \left(\sum_{s, t \neq s \in \mathscr{V}} \frac{\mu_{\mathscr{A}_{2}}(\mathscr{H}(s)) \mu_{\mathscr{A}_{2}}(\mathscr{H}(t))}{\mu_{\mathscr{A}}(\mathscr{H}(s))+\mu_{\mathscr{A}_{2}}(\mathscr{H}(t))-\mu_{\mathscr{A}_{2}}(\mathscr{H}(s)) \mu_{\mathscr{A}_{2}}(\mathscr{H}(t))}\right. \\
& +\mu_{\mathscr{A}_{2}}(\mathscr{H}(s)), \\
& \sum_{s, t \neq s \in \mathscr{V}} \frac{v_{\mathscr{A}_{2}}(\mathscr{H}(s))+v_{\mathscr{A}_{2}}(\mathscr{H}(t))-2 v_{\mathscr{A}_{2}}(\mathscr{H}(s)) v_{\mathscr{A}_{2}}(\mathscr{H}(t))}{1-v_{\mathscr{A}_{2}}(\mathscr{H}(s)) v_{\mathscr{A}_{2}}(\mathscr{H}(t))} \\
+ & \left.v_{\mathscr{A}_{2}}(\mathscr{H}(s))\right) \\
= & \left(\sum_{s t \in E} \mu_{\mathscr{B}}(\mathscr{H}(s) \mathscr{H}(t))+\mu_{\mathscr{A}}(\mathscr{H}(s)),\right. \\
& \left.\sum_{s t \in E} v_{\mathscr{B}_{2}}(\mathscr{H}(s) \mathscr{H}(t))+v_{\mathscr{A}_{2}}(\mathscr{H}(s))\right) \\
= & \left((\mathscr{T} \mathscr{D})_{\mu_{2}}(\mathscr{H}(s)),(\mathscr{T} \mathscr{D})_{v_{2}}(\mathscr{H}(s))\right) .
\end{aligned}
$$

Therefore, $G_{2}$ is a $\left(\mathscr{K}_{1}, \mathscr{K}_{2}\right)$-totally regular PDFG.

Theorem 5 Let $G=(\mathscr{A}, \mathscr{B})$ be a PDFG on underlying graph $G^{*}=(\mathscr{V}, E)$. If $\mu_{\mathscr{A}}$ and $v_{\mathscr{A}}$ are constant functions, then following statements are equal:

1. $G=(\mathscr{A}, \mathscr{B})$ is a regular $P D F G$.

2. $G=(\mathscr{A}, \mathscr{B})$ is a totally regular $P D F G$.

Proof Assume that $\mu_{\mathscr{A}}$ and $v_{\mathscr{A}}$ are constant functions such that $\mu_{\mathscr{A}}(s)=C_{1}$ and $\nu_{\mathscr{A}}(s)=C_{2}$ for all $s \in \mathscr{V}$. Further, suppose that $G$ is a $\left(\mathscr{R}_{1}, \mathscr{R}_{2}\right)$-regular PDFG, then

$$
\begin{aligned}
(\mathscr{D})_{\mu}(s) & =\sum_{s, t \neq s \in \mathscr{V}} \frac{\mu_{\mathscr{A}}(s) \mu_{\mathscr{A}}(t)}{\mu_{\mathscr{A}}(s)+\mu_{\mathscr{A}}(t)-\mu_{\mathscr{A}}(s) \mu_{\mathscr{A}}(t)}=\mathscr{R}_{1}, \\
(\mathscr{D})_{v}(s) & =\sum_{s, t \neq s \in \mathscr{V}} \frac{v_{\mathscr{A}}(s)+v_{\mathscr{A}}(t)-2 v_{\mathscr{A}}(s) v_{\mathscr{A}}(t)}{1-v_{\mathscr{A}}(s) v_{\mathscr{A}}(t)}=\mathscr{R}_{2} .
\end{aligned}
$$

So the total degree of vertex is given by

$$
\begin{aligned}
(\mathscr{T} \mathscr{D})_{\mu}(s)= & \sum_{s, t \neq s \in \mathscr{V}} \frac{\mu_{\mathscr{A}}(s) \mu_{\mathscr{A}}(t)}{\mu_{\mathscr{A}}(s)+\mu_{\mathscr{A}}(t)-\mu_{\mathscr{A}}(s) \mu_{\mathscr{A}}(t)} \\
& +\mu_{\mathscr{A}}(s)=\mathscr{R}_{1}+C_{1},
\end{aligned}
$$

$$
\begin{aligned}
(\mathscr{T} \mathscr{D})_{v}(s)= & \sum_{s, t \neq s \in \mathscr{V}} \frac{v_{\mathscr{A}}(s)+v_{\mathscr{A}}(t)-2 v_{\mathscr{A}}(s) v_{\mathscr{A}}(t)}{1-v_{\mathscr{A}}(s) v_{\mathscr{A}}(t)} \\
& +v_{\mathscr{A}}(s)=\mathscr{R}_{2}+C_{2} .
\end{aligned}
$$

Hence $G$ is a regular PDFG. Therefore, (1) $\Rightarrow(2)$ is proved.

Now suppose that $G$ is a $\left(\mathscr{K}_{1}, \mathscr{K}_{2}\right)$-totally regular PDFG, then

$$
\begin{aligned}
& (\mathscr{T} \mathscr{D})_{\mu}(s)=\mathscr{K}_{1} \\
& \sum_{s, t \neq s \in \mathscr{V}} \frac{\mu_{\mathscr{A}}(s) \mu_{\mathscr{A}}(t)}{\mu_{\mathscr{A}}(s)+\mu_{\mathscr{A}}(t)-\mu_{\mathscr{A}}(s) \mu_{\mathscr{A}}(t)}+\mu_{\mathscr{A}}(s)=\mathscr{K}_{1} \\
& \sum_{s, t \neq s \in \mathscr{V}} \frac{\mu_{\mathscr{A}}(s) \mu_{\mathscr{A}}(t)}{\mu_{\mathscr{A}}(s)+\mu_{\mathscr{A}}(t)-\mu_{\mathscr{A}}(s) \mu_{\mathscr{A}}(t)}+C_{1}=\mathscr{K}_{1} \\
& \sum_{s, t \neq s \in \mathscr{V}} \frac{\mu_{\mathscr{A}}(s) \mu_{\mathscr{A}}(t)}{\mu_{\mathscr{A}}(s)+\mu_{\mathscr{A}}(t)-\mu_{\mathscr{A}}(s) \mu_{\mathscr{A}}(t)}=\mathscr{K}_{1}-C_{1} \\
& (\mathscr{D})_{\mu}(s)=\mathscr{K}_{1}-C_{1} .
\end{aligned}
$$

Likewise, for non-membership grade

$$
\begin{aligned}
& (\mathscr{T} \mathscr{D})_{v}(s)=\mathscr{K}_{2} \\
& \sum_{s, t \neq s \in \mathscr{V}} \frac{v_{\mathscr{A}}(s)+v_{\mathscr{A}}(t)-2 v_{\mathscr{A}}(s) v_{\mathscr{A}}(t)}{1-v_{\mathscr{A}}(s) v_{\mathscr{A}}(t)}+v_{\mathscr{A}}(s)=\mathscr{K}_{2}
\end{aligned}
$$

$\sum_{s, t \neq s \in \mathscr{V}} \frac{v_{\mathscr{A}}(s)+v_{\mathscr{A}}(t)-2 v_{\mathscr{A}}(s) v_{\mathscr{A}}(t)}{1-v_{\mathscr{A}}(s) v_{\mathscr{A}}(t)}+C_{2}=\mathscr{K}_{2}$

$\sum_{s, t \neq s \in \mathscr{V}} \frac{v_{\mathscr{A}}(s)+v_{\mathscr{A}}(t)-2 v_{\mathscr{A}}(s) v_{\mathscr{A}}(t)}{1-v_{\mathscr{A}}(s) v_{\mathscr{A}}(t)}=\mathscr{K}_{2}-C_{2}$

$(\mathscr{D})_{v}(s)=\mathscr{K}_{2}-C_{2}$.

So $G$ is a totally regular PDFG. Therefore, $(2) \Rightarrow(1)$ is proved. Hence we conclude that (1) and (2) are equal.

Theorem 6 Let $G=(\mathscr{A}, \mathscr{B})$ be a PDFG on underlying graph $G^{*}=(\mathscr{V}, E)$. If $G$ is $\left(\mathscr{R}_{1}, \mathscr{R}_{2}\right)$-regular as well as $\left(\mathscr{K}_{1}, \mathscr{K}_{2}\right)$-totally regular PDFG, then $\mu_{\mathscr{A}}$ and $v_{\mathscr{A}}$ are constant functions.

Proof Assume that $G$ is a $\left(\mathscr{R}_{1}, \mathscr{R}_{2}\right)$-regular and $\left(\mathscr{K}_{1}, \mathscr{K}_{2}\right)$ totally regular PDFG. Then the degree of vertex is

$$
\begin{aligned}
& (\mathscr{D})_{\mu}(s)=\sum_{s, t \neq s \in \mathscr{Y}} \frac{\mu_{\mathscr{A}}(s) \mu_{\mathscr{A}}(t)}{\mu_{\mathscr{A}}(s)+\mu_{\mathscr{A}}(t)-\mu_{\mathscr{A}}(s) \mu_{\mathscr{A}}(t)}=\mathscr{R}_{1}, \\
& (\mathscr{D})_{v}(s)=\sum_{s, t \neq s \in \mathscr{Y}} \frac{v_{\mathscr{A}}(s)+v_{\mathscr{A}}(t)-2 v_{\mathscr{A}}(s) v_{\mathscr{A}}(t)}{1-v_{\mathscr{A}}(s) v_{\mathscr{A}}(t)}=\mathscr{R}_{2},
\end{aligned}
$$


and the total degree of vertex is

$$
\begin{aligned}
(\mathscr{T} \mathscr{D})_{\mu}(s)= & \sum_{s, t \neq s \in \mathscr{V}} \frac{\mu_{\mathscr{A}}(s) \mu_{\mathscr{A}}(t)}{\mu_{\mathscr{A}}(s)+\mu_{\mathscr{A}}(t)-\mu_{\mathscr{A}}(s) \mu_{\mathscr{A}}(t)} \\
& +\mu_{\mathscr{A}}(s)=\mathscr{K}_{1}, \\
(\mathscr{T} \mathscr{D})_{\nu}(s)= & \sum_{s, t \neq s \in \mathscr{V}} \frac{v_{\mathscr{A}}(s)+v_{\mathscr{A}}(t)-2 v_{\mathscr{A}}(s) v_{\mathscr{A}}(t)}{1-v_{\mathscr{A}}(s) v_{\mathscr{A}}(t)} \\
& +v_{\mathscr{A}}(s)=\mathscr{K}_{2},
\end{aligned}
$$

Further, it follows that

$$
\begin{aligned}
& (\mathscr{T} \mathscr{D})_{\mu}(s)=\mathscr{K}_{1} \\
& \sum_{s, t \neq s \in \mathscr{V}} \frac{\mu_{\mathscr{A}}(s) \mu_{\mathscr{A}}(t)}{\mu_{\mathscr{A}}(s)+\mu_{\mathscr{A}}(t)-\mu_{\mathscr{A}}(s) \mu_{\mathscr{A}}(t)}+\mu_{\mathscr{A}}(s)=\mathscr{K}_{1} \\
& \mathscr{R}_{1}+\mu_{\mathscr{A}}(s)=\mathscr{K}_{1} \\
& \mu_{\mathscr{A}}(s)=\mathscr{K}_{1}-\mathscr{R}_{1} .
\end{aligned}
$$

Likewise, for non-membership grade

$$
\begin{aligned}
& (\mathscr{T} \mathscr{D})_{v}(s)=\mathscr{K}_{2} \\
& \quad \sum_{s, t \neq s \in \mathscr{V}} \frac{v_{\mathscr{A}}(s)+v_{\mathscr{A}}(t)-2 v_{\mathscr{A}}(s) v_{\mathscr{A}}(t)}{1-v_{\mathscr{A}}(s) v_{\mathscr{A}}(t)}+v_{\mathscr{A}}(s)=\mathscr{K}_{2} \\
& \mathscr{R}_{2}+v_{\mathscr{A}}(s)=\mathscr{K}_{2} \\
& v_{\mathscr{A}}(s)=\mathscr{K}_{2}-\mathscr{R}_{2} .
\end{aligned}
$$

Hence we conclude that $\mu_{\mathscr{A}}=\mathscr{K}_{1}-\mathscr{R}_{1}$ and $v_{\mathscr{A}}=\mathscr{K}_{2}-\mathscr{R}_{2}$ are constant functions.

Remark 3 Converse of the Theorem 6 need not to be true as seen in the following example:

Consider a graph $G^{*}=(\mathscr{V}, E)$, where $\mathscr{V}=\left\{t_{1}, t_{2}, t_{3}\right\}$ and $E=\left\{t_{1} t_{2}, t_{2} t_{3}\right\}$. Let $\mathscr{A}$ and $\mathscr{B}$ be Pythagorean Dombi fuzzy vertex set and Pythagorean Dombi fuzzy edge set defined on $\mathscr{V}$ and $E$, respectively.

$$
\begin{aligned}
\mathscr{A}= & \left\langle\left(\frac{t_{1}}{0.50}, \frac{t_{2}}{0.50}, \frac{t_{3}}{0.50}\right),\right. \\
& \left.\left(\frac{t_{1}}{0.60}, \frac{t_{2}}{0.60}, \frac{t_{3}}{0.60}\right)\right\rangle \text { and } \\
\mathscr{B}= & \left\langle\left(\frac{t_{1} t_{2}}{0.32}, \frac{t_{2} t_{3}}{0.30}\right),\left(\frac{t_{1} t_{2}}{0.70}, \frac{t_{2} t_{3}}{0.75}\right)\right\rangle .
\end{aligned}
$$

Since $\mu_{\mathscr{A}}\left(t_{i}\right)$ and $v_{\mathscr{A}}\left(t_{i}\right)$ are constant functions, where $i=1,2,3$. But $(\mathscr{D})_{G}\left(t_{2}\right)=(0.32,0.70) \neq(0.30,0.75)=$ $(\mathscr{D})_{G}\left(t_{3}\right)$ and $(\mathscr{T} \mathscr{D})_{G}\left(t_{2}\right)=(0.82,1.30) \neq(0.80,1.35)=$ $(\mathscr{T} \mathscr{D})_{G}\left(t_{3}\right)$. Hence $G=(\mathscr{A}, \mathscr{B})$ in Fig. 10 is neither regular nor totally regular PDFG.

Definition 27 A PDFG $G=(\mathscr{A}, \mathscr{B})$ on $n$ vertices is said to be strongly regular if the following properties are satisfied:

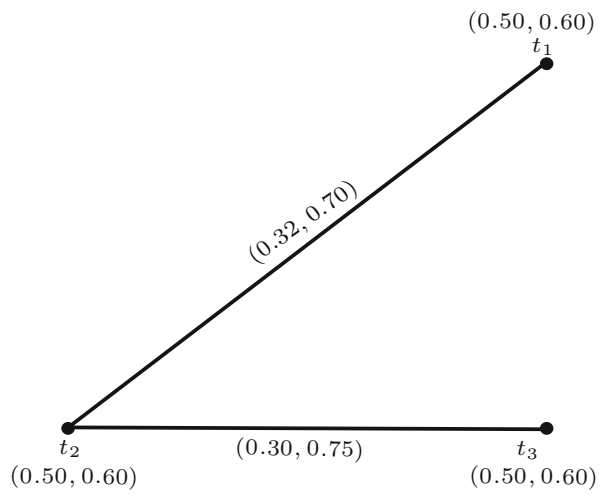

Fig. $10 G=(\mathscr{A}, \mathscr{B})$

1. $G$ is regular of degree $\mathscr{R}=\left(\mathscr{R}_{1}, \mathscr{R}_{2}\right)$;

2 . the sum of the membership and non-membership grades of the common neighbouring vertices of any pair of adjoining vertices of $G$ are equal and represented as $U=\left(U_{1}, U_{2}\right)$;

3. the sum of the membership and non-membership grades of the common neighbouring vertices of any pair of nonadjoining vertices of $G$ are equal and represented as $L=$ $\left(L_{1}, L_{2}\right)$.

A strongly regular PDFG $G=(\mathscr{A}, \mathscr{B})$ is represented as $G=(n, \mathscr{R}, U, L)$.

Example 10 Consider a graph $G^{*}=(\mathscr{V}, E)$, where $\mathscr{V}=$ $\left\{t_{1}, t_{2}, t_{3}, t_{4}, t_{5}, t_{6}\right\}$ and $E=\left\{t_{1} t_{2}, t_{1} t_{3}, t_{1} t_{4}, t_{1} t_{5}, t_{2} t_{3}, t_{2} t_{5}\right.$, $\left.t_{2} t_{6}, t_{3} t_{4}, t_{3} t_{6}, t_{4} t_{5}, t_{4} t_{6}, t_{5} t_{6}\right\}$. Let $\mathscr{A}$ and $\mathscr{B}$ be Pythagorean Dombi fuzzy vertex set and Pythagorean Dombi fuzzy edge set defined on $\mathscr{V}$ and $E$, respectively.

$$
\begin{aligned}
\mathscr{A}= & \left\langle\left(\frac{t_{1}}{0.30}, \frac{t_{2}}{0.30}, \frac{t_{3}}{0.30}, \frac{t_{4}}{0.50}, \frac{t_{5}}{0.50}, \frac{t_{6}}{0.50}\right),\right. \\
& \left.\left(\frac{t_{1}}{0.90}, \frac{t_{2}}{0.90}, \frac{t_{3}}{0.90}, \frac{t_{4}}{0.60}, \frac{t_{5}}{0.60}, \frac{t_{6}}{0.60}\right)\right\rangle \text { and } \\
\mathscr{B}= & \left\langle\left(\frac{t_{1} t_{2}}{0.15}, \frac{t_{1} t_{3}}{0.15}, \frac{t_{1} t_{4}}{0.20}, \frac{t_{1} t_{5}}{0.20}, \frac{t_{2} t_{3}}{0.15}, \frac{t_{2} t_{5}}{0.20},\right.\right. \\
& \left.\frac{t_{2} t_{6}}{0.20}, \frac{t_{3} t_{4}}{0.20}, \frac{t_{3} t_{6}}{0.20}, \frac{t_{4} t_{5}}{0.15}, \frac{t_{4} t_{6}}{0.15}, \frac{t_{5} t_{6}}{0.15}\right), \\
& \left(\frac{t_{1} t_{2}}{0.70}, \frac{t_{1} t_{3}}{0.70}, \frac{t_{1} t_{4}}{0.65}, \frac{t_{1} t_{5}}{0.65}, \frac{t_{2} t_{3}}{0.70}, \frac{t_{2} t_{5}}{0.65},\right. \\
& \left.\left.\frac{t_{2} t_{6}}{0.65}, \frac{t_{3} t_{4}}{0.65}, \frac{t_{3} t_{6}}{0.65}, \frac{t_{4} t_{5}}{0.70}, \frac{t_{4} t_{6}}{0.70}, \frac{t_{5} t_{6}}{0.70}\right)\right\rangle .
\end{aligned}
$$

Since $(\mathscr{D})_{G}\left(t_{i}\right)=(0.7,2.7)$ for all $i=1,2, \ldots, 6$, $G$ is a $(0.7,2.7)$-regular PDFG. Meanwhile, the sum of the membership and non-membership grades of the common neighbouring vertices of each pair of the adjoining and non-adjoining vertices of $G$ are equal, i.e., $U=$ 


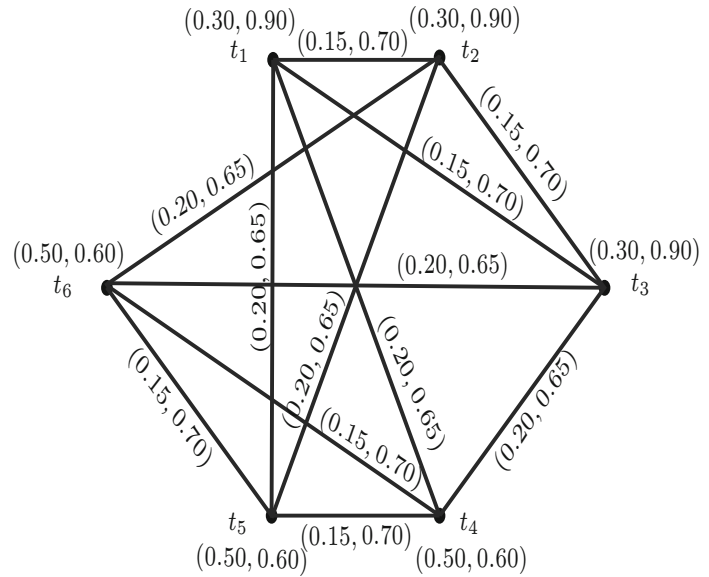

Fig. 11 Strongly regular Pythagorean Dombi fuzzy graph

$\left(U_{1}, U_{2}\right)=(0.8,1)$ and $L=\left(L_{1}, L_{2}\right)=(1.6,2)$. Hence $G=(n, \mathscr{R}, U, L)$ in Fig. 11 is a strongly regular PDFG.

Definition 28 A PDFG $G=(\mathscr{A}, \mathscr{B})$ is known as bipartite if $\mathscr{V}=\left\{s_{1}, s_{2}, \ldots, s_{n}\right\}$ can be partitioned into two nonempty disjoint sets $\mathscr{V}_{1}$ and $\mathscr{V}_{2}$ such that $\mu_{\mathscr{B}}\left(s_{j} s_{k}\right)=0$ and $v_{\mathscr{B}}\left(s_{j} s_{k}\right)=0$ if $s_{j}, s_{k} \in \mathscr{V}_{1}$ or $s_{j}, s_{k} \in \mathscr{V}_{2}$. Furthermore, if

$$
\begin{aligned}
\mu_{\mathscr{B}}\left(s_{j} s_{k}\right) & =\frac{\mu_{\mathscr{A}}\left(s_{j}\right) \mu_{\mathscr{A}}\left(s_{k}\right)}{\mu_{\mathscr{A}}\left(s_{j}\right)+\mu_{\mathscr{A}}\left(s_{k}\right)-\mu_{\mathscr{A}}\left(s_{j}\right) \mu_{\mathscr{A}}\left(s_{k}\right)}, \\
v_{\mathscr{B}}\left(s_{j} s_{k}\right) & =\frac{v_{\mathscr{A}}\left(s_{j}\right)+v_{\mathscr{A}}\left(s_{k}\right)-2 v_{\mathscr{A}}\left(s_{j}\right) \nu_{\mathscr{A}}\left(s_{k}\right)}{1-v_{\mathscr{A}}\left(s_{j}\right) v_{\mathscr{A}}\left(s_{k}\right)} \\
\text { for all } s_{j} & \in \mathscr{V}_{1} \text { and } s_{k} \in \mathscr{V}_{2},
\end{aligned}
$$

then $G=(\mathscr{A}, \mathscr{B})$ is known as complete bipartite, repre-

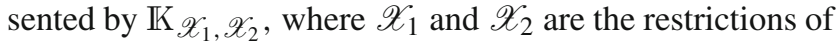
$\mathscr{X}$ to $\mathscr{V}_{1}$ and $\mathscr{X}$ to $\mathscr{V}_{2}$, respectively.

Definition 29 A bipartite PDFG $G=(\mathscr{A}, \mathscr{B})$ is known as biregular if each vertex in $\mathscr{V}_{1}$ and $\mathscr{V} / 2$ have equal degree $\Psi=$ $\left(\Psi_{1}, \Psi_{2}\right)$ and $\Upsilon=\left(\Upsilon_{1}, \Upsilon_{2}\right)$, respectively, where $\Psi$ and $\Upsilon$ are constants. Further, a biregular PDFG is represented by $G=(n, \Psi, \Upsilon)$.

Example 11 Consider a graph $G^{*}=(\mathscr{V}, E)$ as displayed in Fig. 12, where $\mathscr{V}=\left\{t_{1}, t_{2}, t_{3}, t_{4}, t_{5}\right\}$ is partitioned into two non-empty disjoint sets $\mathscr{V}_{1}=\left\{t_{1}, t_{2}, t_{3}\right\}$ and $\mathscr{V} / 2=\left\{t_{4}, t_{5}\right\}$ such that $E=\left\{t_{1} t_{4}, t_{1} t_{5}, t_{2} t_{4}, t_{2} t_{5}, t_{3} t_{4}, t_{3} t_{5}\right\}$.

Since each vertex in $\mathscr{V}_{1}$ and $\mathscr{V}_{2}$ have equal degree $\Psi=$ $\left(\Psi_{1}, \Psi_{2}\right)=(0.55,1.48)$ and $\Upsilon=\left(\Upsilon_{1}, \Upsilon_{2}\right)=(0.83,2.22)$, respectively, $G=(n, \Psi, \Upsilon)$ in Fig. 12, is a biregular PDFG.

Theorem 7 Let $G=(\mathscr{A}, \mathscr{B})$ be a PDFG. If $G$ is complete with $\mu_{\mathscr{A}}, v_{\mathscr{A}}, \mu_{\mathscr{B}}, v_{\mathscr{B}}$ as constant functions, then $G=$ $(\mathscr{A}, \mathscr{B})$ is strongly regular $P D F G$.

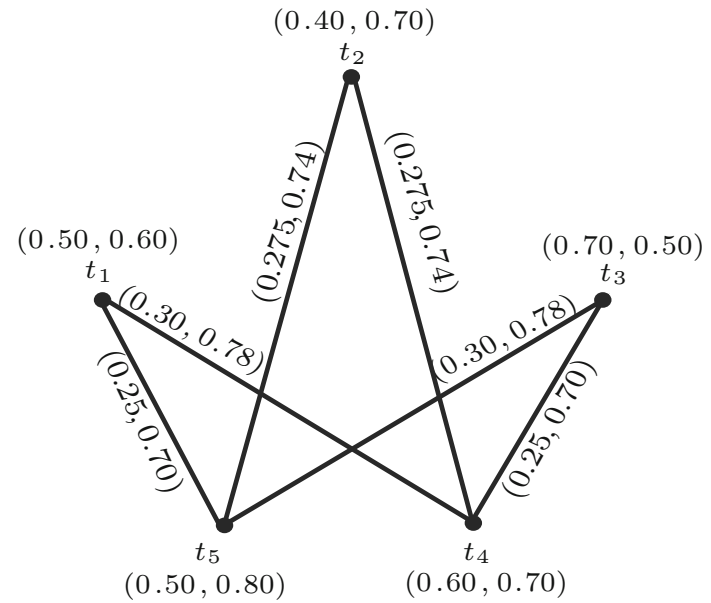

Fig. 12 Biregular Pythagorean Dombi fuzzy graph

Proof Assume that $G$ is a complete PDFG with $\mathscr{V}=$ $\left\{s_{1}, s_{2}, \ldots s_{n}\right\}$. As $\mu_{\mathscr{A}}, v_{\mathscr{A}}, \mu_{\mathscr{B}}$ and $\nu_{\mathscr{B}}$ are constant functions, $\mu_{\mathscr{A}}\left(s_{j}\right)=C_{1}, v_{\mathscr{A}}\left(s_{j}\right)=C_{2}$ for all $s_{j} \in \mathscr{V}$ and $\mu_{\mathscr{B}}\left(s_{j} s_{k}\right)=C_{3}, v_{\mathscr{B}}\left(s_{j} s_{k}\right)=C_{4}$ for all $s_{j} s_{k} \in E$. To prove that $G=(\mathscr{A}, \mathscr{B})$ is a strongly regular PDFG, we must show that $G$ is $\left(\mathscr{R}_{1}, \mathscr{R}_{2}\right)$-regular. Further, the adjoining and non-adjoining vertices have equal common neighbourhood $U=\left(U_{1}, U_{2}\right)$ and $L=\left(L_{1}, L_{2}\right)$, respectively.

As $G$ is a complete PDFG, we must have

$$
\begin{aligned}
(\mathscr{D})_{G}\left(s_{j}\right)= & \left((\mathscr{D})_{\mu}\left(s_{j}\right),(\mathscr{D})_{v}\left(s_{j}\right)\right) \\
= & \left(\sum_{s_{j} s_{k} \in E} \mu_{\mathscr{B}}\left(s_{j} s_{k}\right), \sum_{s_{j} s_{k} \in E} v_{\mathscr{B}}\left(s_{j} s_{k}\right)\right) \\
= & \left(\sum_{s_{j}, s_{k} \neq s_{j} \in \mathscr{V}} \frac{\mu_{\mathscr{A}}\left(s_{j}\right) \mu_{\mathscr{A}}\left(s_{k}\right)}{\mu_{\mathscr{A}}\left(s_{j}\right)+\mu_{\mathscr{A}}\left(s_{k}\right)-\mu_{\mathscr{A}}\left(s_{j}\right) \mu_{\mathscr{A}}\left(s_{k}\right)},\right. \\
& \left.\sum_{s_{j}, s_{k} \neq s_{j} \in \mathscr{V}} \frac{v_{\mathscr{A}}\left(s_{j}\right)+v_{\mathscr{A}}\left(s_{k}\right)-2 v_{\mathscr{A}}\left(s_{j}\right) v_{\mathscr{A}}\left(s_{k}\right)}{1-v_{\mathscr{A}}\left(s_{j}\right) v_{\mathscr{A}}\left(s_{k}\right)}\right) \\
= & \left((n-1) C_{3},(n-1) C_{4}\right) .
\end{aligned}
$$

Hence $G$ is a $\left((n-1) C_{3},(n-1) C_{4}\right)$-regular PDFG. Further, the sum of the membership and non-membership grades of the common neighbouring vertices of any pair of adjoining vertices $U=\left(U_{1}, U_{2}\right)=\left((n-2) C_{1},(n-2) C_{2}\right)$ are equal. As $G$ is complete, the sum of the membership and nonmembership grades of the common neighbouring vertices of any pair of non-adjoining vertices $L=\left(L_{1}, L_{2}\right)=(0,0)$ are equal. Since all the properties are satisfied, we conclude that $G=(\mathscr{A}, \mathscr{B})$ is a strongly regular PDFG.

Remark 4 If $G=(\mathscr{A}, \mathscr{B})$ is a strongly regular disconnected PDFG, then the sum of the membership and non-membership grades of the common neighbouring vertices of any pair of non-adjoining vertices is $L=\left(L_{1}, L_{2}\right)=(0,0)$. 
Remark 5 If $G=(\mathscr{A}, \mathscr{B})$ is a strongly regular complete bipartite PDFG with equal bipartition, then the sum of the membership and non-membership grades of the common neighbouring vertices of any pair of non-adjoining vertices is $L=\left(L_{1}, L_{2}\right)=(0,0)$.

Theorem 8 Let $G=(\mathscr{A}, \mathscr{B})$ be a PDFG. If $G$ is strongly regular and strong, then $\bar{G}$ is a $\left(\mathscr{R}_{1}, \mathscr{R}_{2}\right)$-regular PDFG.

Proof Assume that $G$ is strongly regular PDFG; then by definition $G$ is $\left(\mathscr{R}_{1}, \mathscr{R}_{2}\right)$-regular. Further, as $G$ is strong, then $\bar{G}$ is also strong. Therefore, we must have

$$
\begin{aligned}
& \overline{\mu_{\mathscr{B}}\left(s_{j} s_{k}\right)} \\
& \quad= \begin{cases}\frac{\mu_{\mathscr{A}}\left(s_{j}\right) \mu_{\mathscr{A}}\left(s_{k}\right)}{\mu_{\mathscr{A}}\left(s_{j}\right)+\mu_{\mathscr{A}}\left(s_{k}\right)-\mu_{\mathscr{A}}\left(s_{j}\right) \mu_{\mathscr{A}}\left(s_{k}\right)}, & \text { if } \mu_{\mathscr{B}}\left(s_{j} s_{k}\right)=0, \\
0, & \text { if } 0<\mu_{\mathscr{B}}\left(s_{j} s_{k}\right) \leq 1 .\end{cases} \\
& \overline{\nu_{\mathscr{B}}\left(s_{j} s_{k}\right)} \\
& = \begin{cases}\frac{v_{\mathscr{A}}\left(s_{j}\right)+v_{\mathscr{A}}\left(s_{k}\right)-2 \nu_{\mathscr{A}}\left(s_{j}\right) \nu_{\mathscr{A}}\left(s_{k}\right)}{1-v_{\mathscr{A}}\left(s_{j}\right) \nu_{\mathscr{A}}\left(s_{k}\right)}, & \text { if } \nu_{\mathscr{B}}\left(s_{j} s_{k}\right)=0, \\
0, & \text { if } 0<v_{\mathscr{B}}\left(s_{j} s_{k}\right) \leq 1 .\end{cases}
\end{aligned}
$$

In $\bar{G}$, the degree of a vertex $s_{j}$ is defined by

$$
\begin{aligned}
(\mathscr{D})_{\bar{G}}\left(s_{j}\right)= & \left((\mathscr{D})_{\bar{\mu}}\left(s_{j}\right),(\mathscr{D})_{\bar{v}}\left(s_{j}\right)\right) \\
= & \left(\sum_{s_{j} s_{k} \in E} \overline{\mu_{\mathscr{B}}\left(s_{j} s_{k}\right)}, \sum_{s_{j} s_{k} \in E} \overline{v_{\mathscr{B}}\left(s_{j} s_{k}\right)}\right) \\
= & \left(\sum_{s_{j}, s_{k} \neq s_{j} \in \mathscr{V}} \frac{\mu_{\mathscr{A}}\left(s_{j}\right) \mu_{\mathscr{A}}\left(s_{k}\right)}{\mu_{\mathscr{A}}\left(s_{j}\right)+\mu_{\mathscr{A}}\left(s_{k}\right)-\mu_{\mathscr{A}}\left(s_{j}\right) \mu_{\mathscr{A}}\left(s_{k}\right)},\right. \\
& \left.\sum_{s_{j}, s_{k} \neq s_{j} \in \mathscr{V}} \frac{v_{\mathscr{A}}\left(s_{j}\right)+v_{\mathscr{A}}\left(s_{k}\right)-2 v_{\mathscr{A}}\left(s_{j}\right) v_{\mathscr{A}}\left(s_{k}\right)}{1-v_{\mathscr{A}}\left(s_{j}\right) v_{\mathscr{A}}\left(s_{k}\right)}\right) \\
= & \left(\mathscr{R}_{1}, \mathscr{R}_{2}\right) .
\end{aligned}
$$

As $(\mathscr{D})_{\bar{G}}\left(s_{j}\right)=\left(\mathscr{R}_{1}, \mathscr{R}_{2}\right), \bar{G}$ is $\left(\mathscr{R}_{1}, \mathscr{R}_{2}\right)$-regular PDFG.

Corollary 2 Let $G=(\mathscr{A}, \mathscr{B})$ be a PDFG. If $\bar{G}$ is strongly regular and strong, then $G$ is a $\left(\mathscr{R}_{1}, \mathscr{R}_{2}\right)$-regular PDFG.

Theorem 9 Consider $G=(\mathscr{A}, \mathscr{B})$ be a strong PDFG; then $G$ is a strongly regular $P D F G$ if and only if $\bar{G}$ is a strongly regular PDFG.

Proof Suppose that $G$ is a strongly regular PDFG; then by definition, $G$ is $\left(\mathscr{R}_{1}, \mathscr{R}_{2}\right)$-regular. Further, the adjoining and non-adjoining vertices have equal common neighbourhood $U=\left(U_{1}, U_{2}\right)$ and $L=\left(L_{1}, L_{2}\right)$, respectively. To prove that $\bar{G}$ is a strongly regular PDFG, we must show that $\bar{G}$ is $\left(\mathscr{R}_{1}, \mathscr{R}_{2}\right)$-regular. Since $G$ is a strongly regular and strong, then by Theorem $8, \bar{G}$ is $\left(\mathscr{R}_{1}, \mathscr{R}_{2}\right)$-regular. Moreover, assume that $\mathrm{S}_{1}=\left\{\left(s_{j}, s_{k}\right) /\left(s_{j}, s_{k}\right) \in E\right\}$ and $\mathrm{S}_{2}=\left\{\left(s_{j}, s_{k}\right) /\left(s_{j}, s_{k}\right) \notin E\right\}$ be the sets of all adjoining and non-adjoining vertices of $G$, where $s_{j}$ and $s_{k}$ have equal common neighbourhood $U=\left(U_{1}, U_{2}\right)$ and $L=\left(L_{1}, L_{2}\right)$, respectively. Then $\overline{\mathrm{S}_{1}}=\left\{\left(s_{j}, s_{k}\right) /\left(s_{j}, s_{k}\right) \in \bar{E}\right\}$ and $\overline{\mathrm{S}_{2}}=$ $\left\{\left(s_{j}, s_{k}\right) /\left(s_{j}, s_{k}\right) \notin \bar{E}\right\}$, where $s_{j}$ and $s_{k}$ have equal common neighbourhood $L=\left(L_{1}, L_{2}\right)$ and $U=\left(U_{1}, U_{2}\right)$, respectively. Hence $\bar{G}$ is strongly regular PDFG.

Conversely, $\bar{G}$ is a strongly regular PDFG; then by definition, $\bar{G}$ is $\left(\mathscr{R}_{1}, \mathscr{R}_{2}\right)$ - regular. Further, the adjoining and non-adjoining vertices have equal common neighbourhood $L=\left(L_{1}, L_{2}\right)$ and $U=\left(U_{1}, U_{2}\right)$, respectively. To prove that $G$ is a strongly regular PDFG, we must show that $G$ is $\left(\mathscr{R}_{1}, \mathscr{R}_{2}\right)$-regular. Since $\bar{G}$ is a strongly regular and strong, then by Corollary $2, G$ is $\left(\mathscr{R}_{1}, \mathscr{R}_{2}\right)$-regular. Furthermore, assume that $\overline{S_{1}}=\left\{\left(s_{j}, s_{k}\right) /\left(s_{j}, s_{k}\right) \in \bar{E}\right\}$ and $\overline{\mathrm{S}_{2}}=\left\{\left(s_{j}, s_{k}\right) /\left(s_{j}, s_{k}\right) \notin \bar{E}\right\}$ be the sets of all adjoining and non-adjoining vertices of $\bar{G}$, where $s_{j}$ and $s_{k}$ have equal common neighbourhood: $L=\left(L_{1}, L_{2}\right)$ and $U=\left(U_{1}, U_{2}\right)$, respectively. Then $\mathrm{S}_{1}=\left\{\left(s_{j}, s_{k}\right) /\left(s_{j}, s_{k}\right) \in E\right\}$ and $\mathrm{S}_{2}=$ $\left\{\left(s_{j}, s_{k}\right) /\left(s_{j}, s_{k}\right) \notin E\right\}$, where $s_{j}$ and $s_{k}$ have equal common neighbourhood $U=\left(U_{1}, U_{2}\right)$ and $L=\left(L_{1}, L_{2}\right)$, respectively. Hence $G$ is strongly regular PDFG.

Theorem 10 Let $G=(\mathscr{A}, \mathscr{B})$ be a PDFG such that the underlying graph $G^{*}=(\mathscr{V}, E)$ is strongly regular graph; then $G$ is strongly regular PDFG if $\mu_{\mathscr{A}}, v_{\mathscr{A}}, \mu_{\mathscr{B}}$, and $v_{\mathscr{B}}$ are constant functions.

Proof Assume that $G^{*}=(n, \mathscr{R}, U, L)$ is a strongly regular graph. Also, assume that $\mu_{\mathscr{A}}(s)=C_{1}, v_{\mathscr{A}}(s)=C_{2}$ for all $s \in \mathscr{V}$ and $\mu_{\mathscr{B}}(s t)=C_{3}, v_{\mathscr{B}}(s t)=C_{4}$ for all $s t \in E$. Then the degree of the vertex $s$ is given by

$$
\begin{aligned}
(\mathscr{D})_{\mu}(s) & =\sum_{s, t \neq s \in \mathscr{V}} \frac{\mu_{\mathscr{A}}(s) \mu_{\mathscr{A}}(t)}{\mu_{\mathscr{A}}(s)+\mu_{\mathscr{A}}(t)-\mu_{\mathscr{A}}(s) \mu_{\mathscr{A}}(t)} \\
& =\sum_{s, t \neq s \in \mathscr{V}} C_{3}=(\mathscr{D})_{G^{*}}(s) C_{3}=\mathscr{R} C_{3}, \\
(\mathscr{D})_{\mathcal{V}}(s) & =\sum_{s, t \neq s \in \mathscr{V}} \frac{v_{\mathscr{A}}(s)+v_{\mathscr{A}}(t)-2 v_{\mathscr{A}}(s) v_{\mathscr{A}}(t)}{1-v_{\mathscr{A}}(s) v_{\mathscr{A}}(t)} \\
& =\sum_{s, t \neq s \in \mathscr{V}} C_{4}=(\mathscr{D})_{G^{*}}(s) C_{4}=\mathscr{R} C_{4} .
\end{aligned}
$$

This implies that $(\mathscr{D})_{G}(s)=\left((\mathscr{D})_{\mu}(s),(\mathscr{D})_{\nu}(s)\right)=$ $\left(\mathscr{R} C_{3}, \mathscr{R} C_{4}\right)$. Therefore, $G$ is a $\left(\mathscr{R} C_{3}, \mathscr{R} C_{4}\right)$-regular PDFG. Likewise, since $\mu_{\mathscr{A}}$ and $v_{\mathscr{A}}$ are constant functions, the parameters are $\left(U C_{1}, U C_{2}\right)$ and $\left(L C_{1}, L C_{2}\right)$. Hence $G$ is a strongly regular PDFG with parameters $\left(\mathscr{R} C_{3}, \mathscr{R} C_{4}\right)$, $\left(U C_{1}, U C_{2}\right)$ and $\left(L C_{1}, L C_{2}\right)$.

Remark 6 Converse of the Theorem 10 need not be true as seen in the example given below:

Consider a graph $G^{*}=(\mathscr{V}, E)$, where $\mathscr{V}=\left\{t_{1}, t_{2}, t_{3}, t_{4}\right\}$ and $E=\left\{t_{1} t_{2}, t_{1} t_{3}, t_{1} t_{4}, t_{2} t_{3}, t_{2} t_{4}, t_{3} t_{4}\right\}$. Let $\mathscr{A}$ and $\mathscr{B}$ be Pythagorean Dombi fuzzy vertex set and Pythagorean Dombi fuzzy edge set defined on $\mathscr{V}$ and $E$, respectively. 


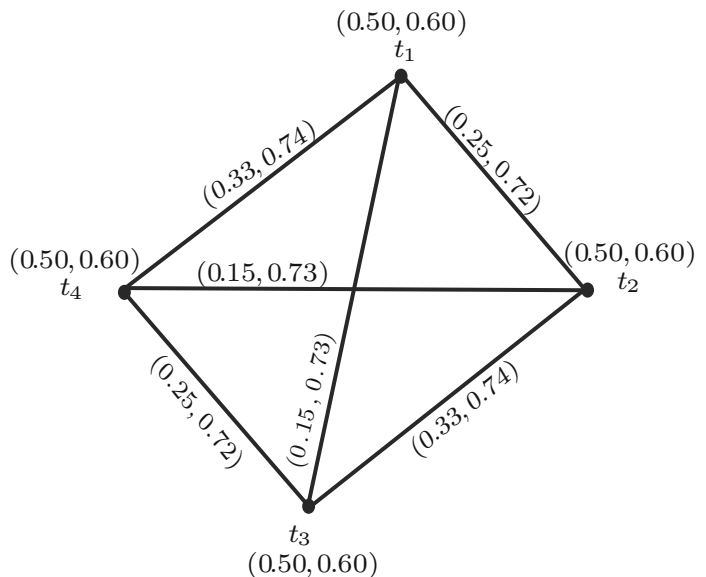

Fig. 13 Pythagorean Dombi fuzzy graph

$$
\begin{aligned}
\mathscr{A}= & \left\langle\left(\frac{t_{1}}{0.50}, \frac{t_{2}}{0.50}, \frac{t_{3}}{0.50}, \frac{t_{4}}{0.50}\right),\right. \\
& \left.\left(\frac{t_{1}}{0.60}, \frac{t_{2}}{0.60}, \frac{t_{3}}{0.60}, \frac{t_{4}}{0.60}\right)\right\rangle \text { and } \\
\mathscr{B}= & \left\langle\left(\frac{t_{1} t_{2}}{0.25}, \frac{t_{1} t_{3}}{0.15}, \frac{t_{1} t_{4}}{0.33}, \frac{t_{2} t_{3}}{0.33}, \frac{t_{2} t_{4}}{0.15}, \frac{t_{3} t_{4}}{0.25}\right),\right. \\
& \left.\left(\frac{t_{1} t_{2}}{0.72}, \frac{t_{1} t_{3}}{0.73}, \frac{t_{1} t_{4}}{0.74}, \frac{t_{2} t_{3}}{0.74}, \frac{t_{2} t_{4}}{0.73}, \frac{t_{3} t_{4}}{0.72}\right)\right\rangle .
\end{aligned}
$$

By routine computations, one can see from Fig. 13 that $G$ is a strongly regular PDFG with parameters $\mathscr{R}=$ $(0.73,2.19), U=(1,0.12)$ and $L=(0,0)$. Meanwhile, $G^{*}$ is a strongly regular graph with parameter $(4,3,2,0)$ and $\mu_{\mathscr{A}}, v_{\mathscr{A}}$ are constant functions. But $\mu_{\mathscr{B}}, v_{\mathscr{B}}$ are not constant functions.

Definition 30 Let $G=(\mathscr{A}, \mathscr{B})$ be a PDFG and $(\mathscr{D})_{1},(\mathscr{D})_{2}$, $\ldots,(\mathscr{D})_{r}$ be the degree of the vertices of $G$. Then the degree sequence is represented by $\left((\mathscr{D})_{1},(\mathscr{D})_{2}, \ldots,(\mathscr{D})_{r}\right)=$ $\left((\mathscr{D})_{\mu_{1}},(\mathscr{D})_{\mu_{2}}, \ldots,(\mathscr{D})_{\mu_{r}} ;(\mathscr{D})_{\nu_{1}},(\mathscr{D})_{\nu_{2}}, \ldots,(\mathscr{D})_{v_{r}}\right)$, where $(\mathscr{D})_{\mu_{1}} \geq(\mathscr{D})_{\mu_{2}} \geq \ldots \geq(\mathscr{D})_{\mu_{r}}$ and $(\mathscr{D})_{\nu_{1}} \geq$ $(\mathscr{D})_{\nu_{2}} \geq \ldots \geq(\mathscr{D})_{\nu_{r}}$.

Definition 31 The set of different positive real values arising in the degree sequence of a PDFG $G$ is known as degree set.

Example 12 Consider a graph $G^{*}=(\mathscr{V}, E)$, as shown in Fig. 14 , where $\mathscr{V}=\left\{t_{1}, t_{2}, \ldots, t_{6}\right\}$ and $E=\left\{t_{1} t_{2}, t_{1} t_{6}, t_{2} t_{5}\right.$, $\left.t_{3} t_{6}, t_{3} t_{4}, t_{4} t_{5}\right\}$. Let $\mathscr{A}$ and $\mathscr{B}$ be Pythagorean Dombi fuzzy vertex set and Pythagorean Dombi fuzzy edge set defined on $\mathscr{V}$ and $E$, respectively.

$$
\begin{aligned}
\mathscr{A}= & \left\langle\left(\frac{t_{1}}{0.50}, \frac{t_{2}}{0.30}, \frac{t_{3}}{0.80}, \frac{t_{4}}{0.70}, \frac{t_{5}}{0.50}, \frac{t_{6}}{0.90}\right),\right. \\
& \left.\left(\frac{t_{1}}{0.60}, \frac{t_{2}}{0.80}, \frac{t_{3}}{0.30}, \frac{t_{4}}{0.40}, \frac{t_{5}}{0.60}, \frac{t_{6}}{0.20}\right)\right\rangle \text { and }
\end{aligned}
$$

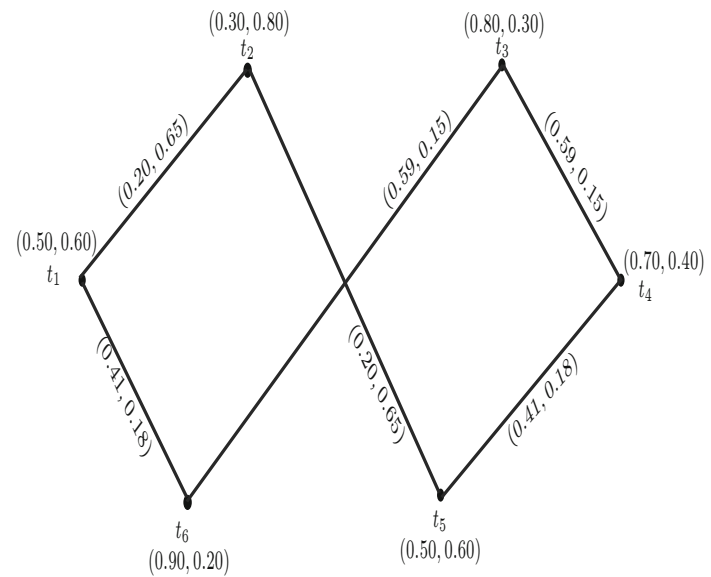

Fig. 14 Pythagorean Dombi fuzzy graph

$$
\begin{aligned}
\mathscr{B}= & \left\langle\left(\frac{t_{1} t_{2}}{0.20}, \frac{t_{1} t_{6}}{0.41}, \frac{t_{2} t_{5}}{0.20}, \frac{t_{3} t_{6}}{0.59}, \frac{t_{3} t_{4}}{0.59}, \frac{t_{4} t_{5}}{0.41}\right),\right. \\
& \left.\left(\frac{t_{1} t_{2}}{0.65}, \frac{t_{1} t_{6}}{0.18}, \frac{t_{2} t_{5}}{0.65}, \frac{t_{3} t_{6}}{0.15}, \frac{t_{3} t_{4}}{0.15}, \frac{t_{4} t_{5}}{0.18}\right)\right\rangle .
\end{aligned}
$$

The degree of the vertices are $(\mathscr{D})_{G}\left(t_{1}\right)=(0.61,0.83)$, $(\mathscr{D})_{G}\left(t_{2}\right)=(0.38,1.25),(\mathscr{D})_{G}\left(t_{3}\right)=(1.29,0.40),(\mathscr{D})_{G}\left(t_{4}\right)$ $=(1,0.33),(\mathscr{D})_{G}\left(t_{5}\right)=(0.59,1.50)$ and $(\mathscr{D})_{G}\left(t_{6}\right)=$ $(1,0.33)$. Hence the degree sequence of the membership grades and the non-membership grades in Fig. 14 are $(1.29,1,1,0.61,0.59,0.38)$ and $(1.25,1.05,0.83,0.40$, $0.33,0.33)$, whereas the corresponding degree sets are $\{1.29,1,0.61,0.59,0.38\}$ and $\{1.25,1.05,0.83,0.40,0.33\}$, respectively.

Theorem 11 Let $G$ be a $(n, \mathscr{R}, U, L)$ strongly regular $P D F G$; then the degree sequence of $n$ elements of $G$ is a constant sequence $\left(\mathscr{R}_{1}, \mathscr{R}_{1}, \ldots, \mathscr{R}_{1} ; \mathscr{R}_{2}, \mathscr{R}_{2}, \ldots, \mathscr{R}_{2}\right)$.

Proof Assume that $G$ is a $(n, \mathscr{R}, U, L)$ strongly regular PDFG; then by definition, $G$ is a $\left(\mathscr{R}_{1}, \mathscr{R}_{2}\right)$-regular. Thus all the vertices have same degree $(\mathscr{D})_{G}\left(s_{i}\right)=\left(\mathscr{R}_{1}, \mathscr{R}_{2}\right)$, where $i=1,2, \ldots, n$. Hence we conclude that the degree sequence of $G$ is a constant sequence $\left(\mathscr{R}_{1}, \mathscr{R}_{1}, \ldots, \mathscr{R}_{1} ; \mathscr{R}_{2}, \mathscr{R}_{2}\right.$, $\left.\ldots, \mathscr{R}_{2}\right)$.

Theorem 12 Let $G$ be a $(n, \mathscr{R}, U, L)$ strongly regular $P D F G$; then the degree set of the membership and non-membership grades of $G$ is a singleton set $\left\{\mathscr{R}_{1}\right\}$ and $\left\{\mathscr{R}_{2}\right\}$, respectively.

Proof Assume that $G$ is a $(n, \mathscr{R}, U, L)$ strongly regular PDFG; then by definition, $G$ is a $\left(\mathscr{R}_{1}, \mathscr{R}_{2}\right)$-regular. Thus all the vertices have same degree $(\mathscr{D})_{G}\left(s_{i}\right)=\left(\mathscr{R}_{1}, \mathscr{R}_{2}\right)$, where $i=1,2, \ldots, n$. As the degree sequence of $G$ is a constant sequence $\left(\mathscr{R}_{1}, \mathscr{R}_{1}, \ldots, \mathscr{R}_{1} ; \mathscr{R}_{2}, \mathscr{R}_{2}, \ldots, \mathscr{R}_{2}\right)$, then the corresponding membership and non-membership degree set is $\left\{\mathscr{R}_{1}\right\}$ and $\left\{\mathscr{R}_{2}\right\}$, respectively. 
Remark 7 Converse of the Theorems 11 and 12 need not be true as seen in the example given below.

Consider a graph $G^{*}=(\mathscr{V}, E)$, where $\mathscr{V}=\left\{t_{1}, t_{2}, t_{3}\right\}$ and $E=\left\{t_{1} t_{2}, t_{1} t_{3}, t_{2} t_{3}\right\}$. Let $\mathscr{A}$ and $\mathscr{B}$ be Pythagorean Dombi fuzzy vertex set and Pythagorean Dombi fuzzy edge set defined on $\mathscr{V}$ and $E$, respectively.

$$
\begin{aligned}
\mathscr{A}= & \left\langle\left(\frac{t_{1}}{0.40}, \frac{t_{2}}{0.40}, \frac{t_{3}}{0.50}\right),\right. \\
& \left.\left(\frac{t_{1}}{0.70}, \frac{t_{2}}{0.70}, \frac{t_{3}}{0.70}\right)\right\rangle \text { and } \\
\mathscr{B}= & \left\langle\left(\frac{t_{1} t_{2}}{0.25}, \frac{t_{1} t_{3}}{0.25}, \frac{t_{2} t_{3}}{0.25}\right),\right. \\
& \left.\left(\frac{t_{1} t_{2}}{0.65}, \frac{t_{1} t_{3}}{0.65}, \frac{t_{2} t_{3}}{0.65}\right)\right\rangle .
\end{aligned}
$$

By routine computations, one can see from Fig. 15 that $G$ has constant membership and non-membership degree sequence $(0.5,0.5,0.5)$ and $(1.3,1.3,1.3)$, respectively, whereas, the corresponding membership and nonmembership degree set is $\{0.5\}$ and $\{1.3\}$, respectively. But $G$ is not strongly regular PDFG as the sum of the membership and non-membership grades of the common neighbouring vertices of any pair of adjoining vertices of $G$ are not equal.

Definition 32 Let $\mathscr{B}=\left\{\left(s t, \mu_{\mathscr{B}}(s t), v_{\mathscr{B}}(s t)\right) \mid s t \in E\right\}$ be a Pythagorean Dombi fuzzy edge set in PDFG $G$; then

- The degree of an edge $s t \in E$ is symbolized by $(\mathscr{D})_{G}(s t)$ and defined by $(\mathscr{D})_{G}(s t)=\left((\mathscr{D})_{\mu}(s t),(\mathscr{D})_{\nu}(s t)\right)$, where

$$
\begin{aligned}
(\mathscr{D})_{\mu}(s t) & =\sum_{s r \in E, t \neq r} \mu_{\mathscr{B}}(s r)+\sum_{t r \in E, s \neq r} \mu_{\mathscr{B}}(t r) \\
& =(\mathscr{D})_{\mu_{\mathscr{A}}}(s)+(\mathscr{D})_{\mu_{\mathscr{A}}}(t)-2 \mu_{\mathscr{B}}(s t)
\end{aligned}
$$

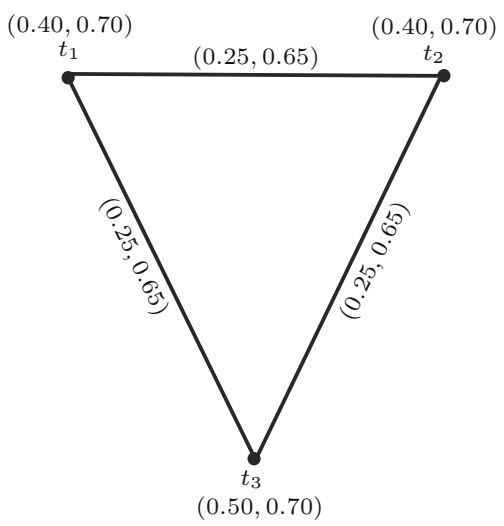

Fig. 15 Pythagorean Dombi fuzzy graph

$$
\begin{aligned}
= & (\mathscr{D})_{\mu_{\mathscr{A}}}(s)+(\mathscr{D})_{\mu_{\mathscr{A}}}(t) \\
& -2\left(\frac{\mu_{\mathscr{A}}(s) \mu_{\mathscr{A}}(t)}{\mu_{\mathscr{A}}(s)+\mu_{\mathscr{A}}(t)-\mu_{\mathscr{A}}(s) \mu_{\mathscr{A}}(t)}\right), \\
(\mathscr{D})_{\nu}(s t)= & \sum_{s r \in E, t \neq r} v_{\mathscr{B}}(s r)+\sum_{t r \in E, s \neq r} v_{\mathscr{B}}(t r) \\
= & (\mathscr{D})_{v_{\mathscr{A}}}(s)+(\mathscr{D})_{v_{\mathscr{A}}}(t)-2 v_{\mathscr{B}}(s t) \\
= & (\mathscr{D})_{v_{\mathscr{A}}}(s)+(\mathscr{D})_{\mathscr{A}}(t) \\
& -2\left(\frac{v_{\mathscr{A}}(s)+v_{\mathscr{A}}(t)-2 v_{\mathscr{A}}(s) v_{\mathscr{A}}(t)}{1-v_{\mathscr{A}}(s) v_{\mathscr{A}}(t)}\right) .
\end{aligned}
$$

- The total degree of an edge st $\in E$ is symbolised by $(\mathscr{T} \mathscr{D})_{G}(s t)$ and defined by $(\mathscr{T} \mathscr{D})_{G}(s t)=$ $\left((\mathscr{T} \mathscr{D})_{\mu}(s t),(\mathscr{T} \mathscr{D})_{v}(s t)\right)$, where

$$
\begin{aligned}
(\mathscr{T} \mathscr{D})_{\mu}(s t)= & \sum_{s r \in E, t \neq r} \mu_{\mathscr{B}}(s r)+\sum_{t r \in E, s \neq r} \mu_{\mathscr{B}}(t r)+\mu_{\mathscr{B}}(s t) \\
= & (\mathscr{D})_{\mu_{\mathscr{A}}}(s)+(\mathscr{D})_{\mu_{\mathscr{A}}}(t)-\mu_{\mathscr{B}}(s t) \\
= & (\mathscr{D})_{\mu_{\mathscr{A}}}(s)+(\mathscr{D})_{\mu_{\mathscr{A}}}(t) \\
& -\left(\frac{\mu_{\mathscr{A}}(s) \mu_{\mathscr{A}}(t)}{\mu_{\mathscr{A}}(s)+\mu_{\mathscr{A}}(t)-\mu_{\mathscr{A}}(s) \mu_{\mathscr{A}}(t)}\right), \\
(\mathscr{T} \mathscr{D})_{v}(s t)= & \sum_{s r \in E, t \neq r} v_{\mathscr{B}}(s r)+\sum_{t r \in E, s \neq r} v_{\mathscr{B}}(t r)+v_{\mathscr{B}}(s t) \\
= & (\mathscr{D})_{v_{\mathscr{A}}}(s)+(\mathscr{D})_{v_{\mathscr{A}}}(t)-v_{\mathscr{B}}(s t) \\
= & (\mathscr{D})_{v_{\mathscr{A}}}(s)+(\mathscr{D})_{v_{\mathscr{A}}}(t) \\
& -\left(\frac{v_{\mathscr{A}}(s)+v_{\mathscr{A}}(t)-2 v_{\mathscr{A}}(s) v_{\mathscr{A}}(t)}{1-v_{\mathscr{A}}(s) v_{\mathscr{A}}(t)}\right) .
\end{aligned}
$$

Example 13 Consider a PDFG $G$ over $\mathscr{V}=\left\{t_{1}, t_{2}, \ldots, t_{13}\right\}$ as displayed in Fig. 16.

Then the degree of an edge $t_{7} t_{8}$ is $(\mathscr{D})_{G}\left(t_{7} t_{8}\right)=$ $\left((\mathscr{D})_{\mu_{\mathscr{A}}}\left(t_{7}\right)+(\mathscr{D})_{\mu_{\mathscr{A}}}\left(t_{8}\right)-2(\mathscr{D})_{\mu_{\mathscr{B}}}\left(t_{7} t_{8}\right),(\mathscr{D})_{\nu_{\mathscr{A}}}\left(t_{7}\right)+\right.$ $\left.(\mathscr{D})_{\nu_{\mathscr{A}}}\left(t_{8}\right)-2(\mathscr{D})_{\nu_{\mathscr{B}}}\left(t_{7} t_{8}\right)\right)=(0.44,1.72)$. Meanwhile, the total degree of an edge $t_{7} t_{8}$ is $(\mathscr{T} \mathscr{D})_{G}\left(t_{7} t_{8}\right)=\left((\mathscr{D})_{\mu_{\mathscr{A}}}\left(t_{7}\right)+\right.$ $(\mathscr{D})_{\mu_{\mathscr{A}}}\left(t_{8}\right)-(\mathscr{D})_{\mu_{\mathscr{B}}}\left(t_{7} t_{8}\right),(\mathscr{D})_{v_{\mathscr{A}}}\left(t_{7}\right)+(\mathscr{D})_{v_{\mathscr{A}}}\left(t_{8}\right)-(\mathscr{D})_{v_{\mathscr{B}}}$ $\left.\left(t_{7} t_{8}\right)\right)=(0.89,2.22)$. Likewise, one can obtain the degree and total degree of all remaining edges in $G$.

Remark 8 If $G=(\mathscr{A}, \mathscr{B})$ is a strongly regular PDFG, then the membership and non-membership degree sequence of edge need not to be constant sequence as seen in the example given below:

Consider a graph $G^{*}=(\mathscr{V}, E)$, where $\mathscr{V}=\left\{t_{1}, t_{2}, t_{3}, t_{4}\right\}$ and $E=\left\{t_{1} t_{2}, t_{1} t_{4}, t_{2} t_{3}, t_{3} t_{4}\right\}$. Let $\mathscr{A}$ and $\mathscr{B}$ be Pythagorean Dombi fuzzy vertex set and Pythagorean Dombi fuzzy edge set defined on $\mathscr{V}$ and $E$, respectively.

$$
\begin{aligned}
\mathscr{A}= & \left\langle\left(\frac{t_{1}}{0.50}, \frac{t_{2}}{0.50}, \frac{t_{3}}{0.50}, \frac{t_{4}}{0.50}\right),\right. \\
& \left.\left(\frac{t_{1}}{0.60}, \frac{t_{2}}{0.60}, \frac{t_{3}}{0.60}, \frac{t_{4}}{0.60}\right)\right\rangle
\end{aligned}
$$




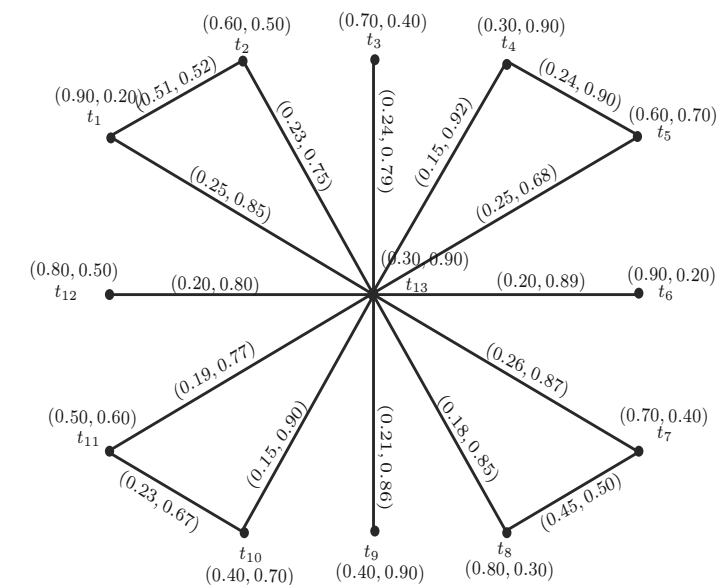

Fig. 16 Pythagorean Dombi fuzzy graph

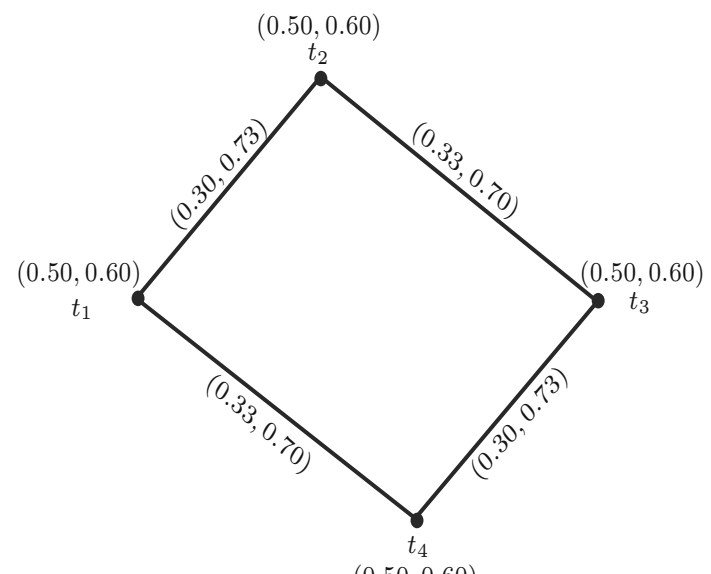

$(0.50,0.60)$

Fig. 17 Pythagorean Dombi fuzzy graph

$$
\begin{aligned}
\mathscr{B}= & \left\langle\left(\frac{t_{1} t_{2}}{0.33}, \frac{t_{1} t_{4}}{0.30}, \frac{t_{2} t_{3}}{0.30}, \frac{t_{3} t_{4}}{0.33}\right),\right. \\
& \left.\left(\frac{t_{1} t_{2}}{0.70}, \frac{t_{1} t_{4}}{0.73}, \frac{t_{2} t_{3}}{0.73}, \frac{t_{3} t_{4}}{0.70}\right)\right\rangle .
\end{aligned}
$$

By routine computations, one can see from Fig. 17 that $G$ is a strongly regular PDFG with parameters $\mathscr{R}=$ $(0.63,1.43), U=(0,0)$ and $L=(1,1.2)$. The edge degree sequence of the membership grades and the non-membership grades is $(0.66,0.66,0.60,0.60)$ and $(1.46,1.46,1.4,1.4)$, respectively, whereas the corresponding edge degree sets $\{0.66,0.60\}$ and $\{1.46,1.4\}$ are not constant sequence.

Theorem 13 Let $G=(\mathscr{A}, \mathscr{B})$ be a strongly regular $P D F G$ with $\mu_{\mathscr{B}}$ and $\nu_{\mathscr{B}}$ as constant functions; then the edge degree sequence and edge degree set are constant sequence and singleton set, respectively.

Proof Assume that $\mu_{\mathscr{B}}=C_{1}$ and $\nu_{\mathscr{B}}=C_{2}$. Also, suppose that $G$ is a strongly regular PDFG; then by definition, $G$ is $\left(\mathscr{R}_{1}, \mathscr{R}_{2}\right)$-regular such that

$$
\begin{aligned}
(\mathscr{D})_{\mu}(s) & =\sum_{s, t \neq s \in \mathscr{V}} \frac{\mu_{\mathscr{A}}(s) \mu_{\mathscr{A}}(t)}{\mu_{\mathscr{A}}(s)+\mu_{\mathscr{A}}(t)-\mu_{\mathscr{A}}(s) \mu_{\mathscr{A}}(t)}=\mathscr{R}_{1}, \\
(\mathscr{D})_{v}(s) & =\sum_{s, t \neq s \in \mathscr{V}} \frac{v_{\mathscr{A}}(s)+v_{\mathscr{A}}(t)-2 v_{\mathscr{A}}(s) v_{\mathscr{A}}(t)}{1-v_{\mathscr{A}}(s) v_{\mathscr{A}}(t)}=\mathscr{R}_{2} .
\end{aligned}
$$

Therefore, the degree of edge is $(\mathscr{D})_{G}(s t)=\left((\mathscr{D})_{\mu}(s t)\right.$, $\left.(\mathscr{D})_{v}(s t)\right)$, whereas

$$
\begin{aligned}
(\mathscr{D})_{\mu}(s t)= & (\mathscr{D})_{\mu_{\mathscr{A}}}(s)+(\mathscr{D})_{\mu_{\mathscr{A}}}(t) \\
& -2\left(\frac{\mu_{\mathscr{A}}(s) \mu_{\mathscr{A}}(t)}{\mu_{\mathscr{A}}(s)+\mu_{\mathscr{A}}(t)-\mu_{\mathscr{A}}(s) \mu_{\mathscr{A}}(t)}\right) \\
= & \mathscr{R}_{1}+\mathscr{R}_{1}-2 C_{1}=2 \mathscr{R}_{1}-2 C_{1}=2\left(\mathscr{R}_{1}-C_{1}\right), \\
(\mathscr{D})_{v}(s t)= & (\mathscr{D})_{v_{\mathscr{A}}}(s)+(\mathscr{D})_{v_{\mathscr{A}}}(t) \\
& -2\left(\frac{v_{\mathscr{A}}(s)+v_{\mathscr{A}}(t)-2 v_{\mathscr{A}}(s) v_{\mathscr{A}}(t)}{1-v_{\mathscr{A}}(s) v_{\mathscr{A}}(t)}\right) \\
= & \mathscr{R}_{2}+\mathscr{R}_{2}-2 C_{2}=2 \mathscr{R}_{2}-2 C_{2}=2\left(\mathscr{R}_{2}-C_{2}\right) .
\end{aligned}
$$

Hence the edge degree sequence of membership and nonmembership grades are constant sequence and its corresponding edge degree set $\left\{2\left(\mathscr{R}_{1}-C_{1}\right)\right\}$ and $\left\{2\left(\mathscr{R}_{2}-C_{2}\right)\right\}$ are singleton sets.

Definition 33 A PDFG $G=(\mathscr{A}, \mathscr{B})$ on crisp graph $G^{*}=$ $(\mathscr{V}, E)$ is said to be edge regular of degree $\left(\mathrm{R}_{1}, \mathrm{R}_{2}\right)$ or $\left(\mathrm{R}_{1}, \mathrm{R}_{2}\right)$-edge regular. If

$$
\begin{aligned}
(\mathscr{D})_{\mu}(s t)= & (\mathscr{D})_{\mu_{\mathscr{A}}}(s)+(\mathscr{D})_{\mu_{\mathscr{A}}}(t)-2 \mu_{\mathscr{B}}(s t) \\
= & (\mathscr{D})_{\mu_{\mathscr{A}}}(s)+(\mathscr{D})_{\mu_{\mathscr{A}}}(t) \\
& -2\left(\frac{\mu_{\mathscr{A}}(s) \mu_{\mathscr{A}}(t)}{\mu_{\mathscr{A}}(s)+\mu_{\mathscr{A}}(t)-\mu_{\mathscr{A}}(s) \mu_{\mathscr{A}}(t)}\right)=\mathrm{R}_{1}, \\
(\mathscr{D})_{v}(s t)= & (\mathscr{D})_{v_{\mathscr{A}}}(s)+(\mathscr{D})_{v_{\mathscr{A}}}(t)-2 v_{\mathscr{B}}(s t) \\
= & (\mathscr{D})_{v_{\mathscr{A}}}(s)+(\mathscr{D})_{v_{\mathscr{A}}}(t) \\
& -2\left(\frac{v_{\mathscr{A}}(s)+v_{\mathscr{A}}(t)-2 v_{\mathscr{A}}(s) v_{\mathscr{A}}(t)}{1-v_{\mathscr{A}}(s) v_{\mathscr{A}}(t)}\right)=\mathrm{R}_{2}
\end{aligned}
$$

for all st $\in \mathrm{E}$.

Example 14 Consider a graph $G^{*}=(\mathscr{V}, E)$, where $\mathscr{V}=$ $\left\{t_{1}, t_{2}, t_{3}, t_{4}\right\}$ and $E=\left\{t_{1} t_{2}, t_{1} t_{4}, t_{2} t_{3}, t_{3} t_{4}\right\}$. Let $\mathscr{A}$ and $\mathscr{B}$ be Pythagorean Dombi fuzzy vertex set and Pythagorean Dombi fuzzy edge set defined on $\mathscr{V}$ and $E$, respectively.

$$
\begin{aligned}
\mathscr{A}= & \left\langle\left(\frac{t_{1}}{0.40}, \frac{t_{2}}{0.20}, \frac{t_{3}}{0.80}, \frac{t_{4}}{0.70}\right),\right. \\
& \left.\left(\frac{t_{1}}{0.80}, \frac{t_{2}}{0.90}, \frac{t_{3}}{0.30}, \frac{t_{4}}{0.40}\right)\right\rangle \text { and } \\
\mathscr{B}= & \left\langle\left(\frac{t_{1} t_{2}}{0.15}, \frac{t_{1} t_{4}}{0.15}, \frac{t_{2} t_{3}}{0.18}, \frac{t_{3} t_{4}}{0.18}\right),\right. \\
& \left.\left(\frac{t_{1} t_{2}}{0.80}, \frac{t_{1} t_{4}}{0.80}, \frac{t_{2} t_{3}}{0.50}, \frac{t_{3} t_{4}}{0.50}\right)\right\rangle .
\end{aligned}
$$




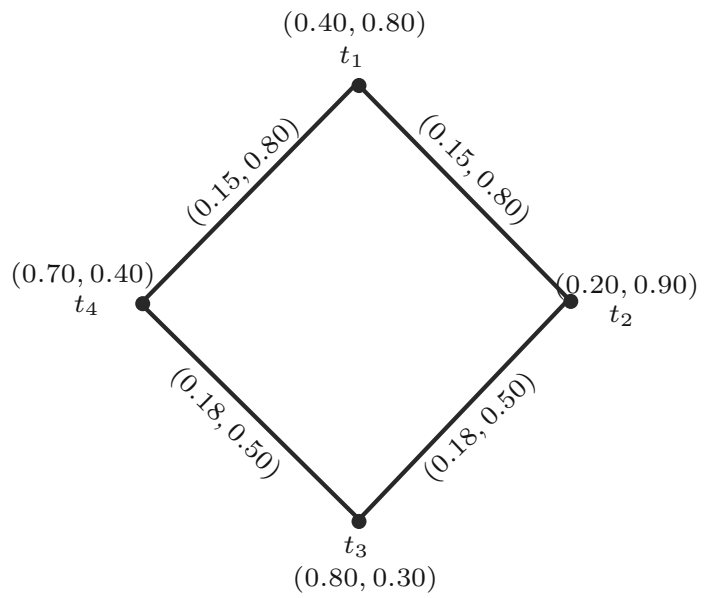

Fig. 18 (0.33, 1.3)-edge regular Pythagorean Dombi fuzzy graph

Since the degree of each edge $(\mathscr{D})_{G}\left(s_{j} s_{k}\right)=(0.33,1.3)$ for all $j, k=1,2,3,4, G$ in Fig. 18, is $(0.33,1.3)$-edge regular PDFG.

Definition 34 A PDFG $G=(\mathscr{A}, \mathscr{B})$ on crisp graph $G^{*}=$ $(\mathscr{V}, E)$ is said to be totally edge regular of degree $\left(\mathrm{K}_{1}, \mathrm{~K}_{2}\right)$ or $\left(\mathrm{K}_{1}, \mathrm{~K}_{2}\right)$-totally edge regular. If

$$
\begin{aligned}
(\mathscr{T} \mathscr{D})_{\mu}(s t)= & (\mathscr{D})_{\mu_{\mathscr{A}}}(s)+(\mathscr{D})_{\mu_{\mathscr{A}}}(t)-\mu_{\mathscr{B}}(s t) \\
= & (\mathscr{D})_{\mu_{\mathscr{A}}}(s)+(\mathscr{D})_{\mu_{\mathscr{A}}}(t) \\
& -\left(\frac{\mu_{\mathscr{A}}(s) \mu_{\mathscr{A}}(t)}{\mu_{\mathscr{A}}(s)+\mu_{\mathscr{A}}(t)-\mu_{\mathscr{A}}(s) \mu_{\mathscr{A}}(t)}\right)=\mathrm{K}_{1}, \\
(\mathscr{T} \mathscr{D})_{v}(s t)= & (\mathscr{D})_{v_{\mathscr{A}}}(s)+(\mathscr{D})_{v_{\mathscr{A}}}(t)-v_{\mathscr{B}}(s t) \\
= & (\mathscr{D})_{v_{\mathscr{A}}}(s)+(\mathscr{D})_{v_{\mathscr{A}}}(t) \\
& -\left(\frac{v_{\mathscr{A}}(s)+v_{\mathscr{A}}(t)-2 v_{\mathscr{A}}(s) v_{\mathscr{A}}(t)}{1-v_{\mathscr{A}}(s) v_{\mathscr{A}}(t)}\right)=\mathrm{K}_{2}
\end{aligned}
$$

for all st $\in \mathrm{E}$.

Example 15 Consider a graph $G^{*}=(\mathscr{V}, E)$, where $\mathscr{V}=$ $\left\{t_{1}, t_{2}, t_{3}, t_{4}\right\}$ and $E=\left\{t_{1} t_{2}, t_{1} t_{3}, t_{1} t_{4}, t_{2} t_{3}, t_{2} t_{4}, t_{3} t_{4}\right\}$. Let $\mathscr{A}$ and $\mathscr{B}$ be Pythagorean Dombi fuzzy vertex set and Pythagorean Dombi fuzzy edge set defined on $\mathscr{V}$ and $E$, respectively.

$$
\begin{aligned}
\mathscr{A}= & \left\langle\left(\frac{t_{1}}{0.70}, \frac{t_{2}}{0.80}, \frac{t_{3}}{0.90}, \frac{t_{4}}{0.50}\right),\right. \\
& \left.\left(\frac{t_{1}}{0.50}, \frac{t_{2}}{0.40}, \frac{t_{3}}{0.20}, \frac{t_{4}}{0.60}\right)\right\rangle \text { and } \\
\mathscr{B}= & \left\langle\left(\frac{t_{1} t_{2}}{0.40}, \frac{t_{1} t_{3}}{0.40}, \frac{t_{1} t_{4}}{0.40}, \frac{t_{2} t_{3}}{0.40}, \frac{t_{2} t_{4}}{0.40}, \frac{t_{3} t_{4}}{0.40}\right),\right. \\
& \left.\left(\frac{t_{1} t_{2}}{0.47}, \frac{t_{1} t_{3}}{0.47}, \frac{t_{1} t_{4}}{0.47}, \frac{t_{2} t_{3}}{0.47}, \frac{t_{2} t_{4}}{0.47}, \frac{t_{3} t_{4}}{0.47}\right)\right\rangle .
\end{aligned}
$$

$(0.80,0.40)$

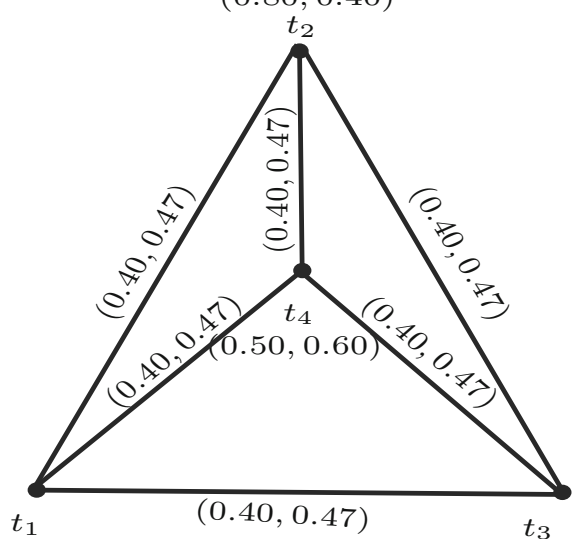

$(0.70,0.50)$

$(0.90,0.20)$

Fig. 19 (0.20, 2.35)-totally edge regular Pythagorean Dombi fuzzy graph

Since the total degree of each edge $(\mathscr{T} \mathscr{D})_{G}\left(s_{j} s_{k}\right)=$ $(0.20,2.35)$ for all $j, k=1,2,3,4, G$ in Fig. 19, is $(0.20,2.35)$-totally edge regular PDFG.

Theorem 14 Let $G=(\mathscr{A}, \mathscr{B})$ be a $\left(\mathscr{R}_{1}, \mathscr{R}_{2}\right)$-regular PDFG such that $\mu_{\mathscr{B}}$ and $v_{\mathscr{B}}$ are constant functions; then $G$ is $\left(\mathrm{R}_{1}, \mathrm{R}_{2}\right)$-edge regular PDFG.

Proof Assume that $G$ is a $\left(\mathscr{R}_{1}, \mathscr{R}_{2}\right)$-regular PDFG such that

$$
\begin{aligned}
& (\mathscr{D})_{\mu}\left(s_{j}\right) \\
& =\sum_{s_{j}, s_{k} \neq s_{j} \in \mathscr{V}} \frac{\mu_{\mathscr{A}}\left(s_{j}\right) \mu_{\mathscr{A}}\left(s_{k}\right)}{\mu_{\mathscr{A}}\left(s_{j}\right)+\mu_{\mathscr{A}}\left(s_{k}\right)-\mu_{\mathscr{A}}\left(s_{j}\right) \mu_{\mathscr{A}}\left(s_{k}\right)}=\mathscr{R}_{1}, \\
& (\mathscr{D})_{v}\left(s_{j}\right) \\
& =\sum_{s_{j}, s_{k} \neq s_{j} \in \mathscr{V}} \frac{v_{\mathscr{A}}\left(s_{j}\right)+v_{\mathscr{A}}\left(s_{k}\right)-2 v_{\mathscr{A}}\left(s_{j}\right) v_{\mathscr{A}}\left(s_{k}\right)}{1-v_{\mathscr{A}}\left(s_{j}\right) v_{\mathscr{A}}\left(s_{k}\right)}=\mathscr{R}_{2} .
\end{aligned}
$$

As $\mu_{\mathscr{B}}$ and $\nu_{\mathscr{B}}$ are constant functions, $\mu_{\mathscr{B}}\left(s_{j} s_{k}\right)=C_{1}$ and $v_{\mathscr{B}}\left(s_{j} s_{k}\right)=C_{2}$ for all $s_{j} s_{k} \in E$. By using the definition of edge degree $(\mathscr{D})_{G}\left(s_{j} s_{k}\right)=\left((\mathscr{D})_{\mu}\left(s_{j} s_{k}\right),(\mathscr{D})_{\nu}\left(s_{j} s_{k}\right)\right)$, whereas

$$
\begin{aligned}
(\mathscr{D})_{\mu}\left(s_{j} s_{k}\right)= & (\mathscr{D})_{\mu_{\mathscr{A}}}\left(s_{j}\right)+(\mathscr{D})_{\mu_{\mathscr{A}}}\left(s_{k}\right) \\
& -2\left(\frac{\mu_{\mathscr{A}}\left(s_{j}\right) \mu_{\mathscr{A}}\left(s_{k}\right)}{\mu_{\mathscr{A}}\left(s_{j}\right)+\mu_{\mathscr{A}}\left(s_{k}\right)-\mu_{\mathscr{A}}\left(s_{j}\right) \mu_{\mathscr{A}}\left(s_{k}\right)}\right) \\
= & \mathscr{R}_{1}+\mathscr{R}_{1}-2 C_{1}=2 \mathscr{R}_{1}-2 C_{1}=2\left(\mathscr{R}_{1}-C_{1}\right)=\mathrm{R}_{1}, \\
(\mathscr{D})_{v}\left(s_{j} s_{k}\right)= & (\mathscr{D})_{v_{\mathscr{A}}}\left(s_{j}\right)+(\mathscr{D})_{v_{\mathscr{A}}}\left(s_{k}\right) \\
& -2\left(\frac{v_{\mathscr{A}}\left(s_{j}\right)+v_{\mathscr{A}}\left(s_{k}\right)-2 \nu_{\mathscr{A}}\left(s_{j}\right) \nu_{\mathscr{A}}\left(s_{k}\right)}{1-v_{\mathscr{A}}\left(s_{j}\right) v_{\mathscr{A}}\left(s_{k}\right)}\right) \\
= & \mathscr{R}_{2}+\mathscr{R}_{2}-2 C_{2}=2 \mathscr{R}_{2}-2 C_{2}=2\left(\mathscr{R}_{2}-C_{2}\right)=\mathrm{R}_{2} .
\end{aligned}
$$

Hence we conclude that $G$ is a $\left(\mathrm{R}_{1}, \mathrm{R}_{2}\right)$-edge regular PDFG. 
Theorem 15 Let $G=(\mathscr{A}, \mathscr{B})$ be both $\left(\mathrm{R}_{1}, \mathrm{R}_{2}\right)$-edge regular and $\left(\mathrm{K}_{1}, \mathrm{~K}_{2}\right)$-totally edge regular PDFG; then $\mu_{\mathscr{B}}$ and $v_{\mathscr{B}}$ are constant functions.

Proof Assume that $G$ is a $\left(\mathrm{R}_{1}, \mathrm{R}_{2}\right)$-edge regular and $\left(\mathrm{K}_{1}\right.$, $\mathrm{K}_{2}$ )-totally edge regular PDFG. Then the degree of edge is

$$
\begin{aligned}
(\mathscr{D})_{\mu}(s t)= & (\mathscr{D})_{\mu_{\mathscr{A}}}(s)+(\mathscr{D})_{\mu_{\mathscr{A}}}(t) \\
& -2\left(\frac{\mu_{\mathscr{A}}(s) \mu_{\mathscr{A}}(t)}{\mu_{\mathscr{A}}(s)+\mu_{\mathscr{A}}(t)-\mu_{\mathscr{A}}(s) \mu_{\mathscr{A}}(t)}\right)=\mathrm{R}_{1}, \\
(\mathscr{D})_{v}(s t)= & (\mathscr{D})_{v_{\mathscr{A}}}(s)+(\mathscr{D})_{v_{\mathscr{A}}}(t) \\
& -2\left(\frac{v_{\mathscr{A}}(s)+v_{\mathscr{A}}(t)-2 v_{\mathscr{A}}(s) v_{\mathscr{A}}(t)}{1-v_{\mathscr{A}}(s) v_{\mathscr{A}}(t)}\right)=\mathrm{R}_{2},
\end{aligned}
$$

and the total degree of edge is

$$
\begin{aligned}
(\mathscr{T} \mathscr{D})_{\mu}(s t)= & (\mathscr{D})_{\mu_{\mathscr{A}}}(s)+(\mathscr{D})_{\mu_{\mathscr{A}}}(t) \\
& -\left(\frac{\mu_{\mathscr{A}}(s) \mu_{\mathscr{A}}(t)}{\mu_{\mathscr{A}}(s)+\mu_{\mathscr{A}}(t)-\mu_{\mathscr{A}}(s) \mu_{\mathscr{A}}(t)}\right)=\mathrm{K}_{1}, \\
(\mathscr{T} \mathscr{D})_{v}(s t)= & (\mathscr{D})_{v_{\mathscr{A}}}(s)+(\mathscr{D})_{\mathscr{\mathscr { A }}}(t) \\
& -\left(\frac{v_{\mathscr{A}}(s)+v_{\mathscr{A}}(t)-v_{\mathscr{A}}(s) v_{\mathscr{A}}(t)}{1-v_{\mathscr{A}}(s) v_{\mathscr{A}}(t)}\right)=\mathrm{K}_{2} .
\end{aligned}
$$

Further, it follows that

$$
\begin{aligned}
& (\mathscr{T} \mathscr{D}) \mu(s t)=\mathrm{K}_{1} \\
& \left(\mathscr{D} \mu_{\mathscr{A}}(s)+(\mathscr{D})_{\mathscr{A}}(t)\right. \\
& \quad-\left(\frac{\mu_{\mathscr{A}}(s) \mu_{\mathscr{A}}(t)}{\mu_{\mathscr{A}}(s)+\mu_{\mathscr{A}}(t)-\mu_{\mathscr{A}}(s) \mu_{\mathscr{A}}(t)}\right)=\mathrm{K}_{1} \\
& (\mathscr{D}) \mu_{\mathscr{A}}(s)+(\mathscr{D})_{\mathscr{A}}(t) \\
& \quad-2\left(\frac{\mu_{\mathscr{A}}(s) \mu_{\mathscr{A}}(t)}{\mu_{\mathscr{A}}(s)+\mu_{\mathscr{A}}(t)-\mu_{\mathscr{A}}(s) \mu_{\mathscr{A}}(t)}\right)+\mu_{\mathscr{B}}(s t)=\mathrm{K}_{1} \\
& \mu_{\mathscr{B}}(s t)=\mathrm{K}_{1}-\mathrm{R}_{1} .
\end{aligned}
$$

Likewise, for non-membership grade

$$
\begin{aligned}
& (\mathscr{T} \mathscr{D})_{v}(s t)=\mathrm{K}_{2} \\
& (\mathscr{D})_{\mathscr{A}}(s)+(\mathscr{D})_{v_{\mathscr{A}}}(t) \\
& \quad-\left(\frac{v_{\mathscr{A}}(s)+v_{\mathscr{A}}(t)-2 v_{\mathscr{A}}(s) v_{\mathscr{A}}(t)}{1-v_{\mathscr{A}}(s) v_{\mathscr{A}}(t)}\right)=\mathrm{K}_{2} \\
& (\mathscr{D})_{v_{\mathscr{A}}}(s)+(\mathscr{D})_{v_{\mathscr{A}}}(t) \\
& \quad-2\left(\frac{v_{\mathscr{A}}(s)+v_{\mathscr{A}}(t)-2 v_{\mathscr{A}}(s) v_{\mathscr{A}}(t)}{1-v_{\mathscr{A}}(s) v_{\mathscr{A}}(t)}\right)+v_{\mathscr{B}}(s t)=\mathrm{K}_{2} \\
& v_{\mathscr{B}}(s t)=\mathrm{K}_{2}-\mathrm{R}_{2} .
\end{aligned}
$$

Hence we conclude that $\mu_{\mathscr{B}}=\mathrm{K}_{1}-\mathrm{R}_{1}$ and $v_{\mathscr{B}}=\mathrm{K}_{2}-\mathrm{R}_{2}$ are constant functions.

Theorem 16 Let $G=(\mathscr{A}, \mathscr{B})$ be a $P D F G$ on a regular crisp graph $G^{*}=(\mathscr{V}, E)$. Then $\mu_{\mathscr{B}}$ and $\nu_{\mathscr{B}}$ are constant functions if and only if $G=(\mathscr{A}, \mathscr{B})$ is both $\left(\mathscr{R}_{1}, \mathscr{R}_{2}\right)$-regular and $\left(\mathrm{K}_{1}, \mathrm{~K}_{2}\right)$-totally edge regular $P D F G$.
Proof Assume that $G$ is a PDFG on a regular crisp graph $G^{*}$. Also, suppose that $\mu_{\mathscr{B}}$ and $\nu_{\mathscr{B}}$ are constant functions; then $\mu_{\mathscr{B}}\left(s_{j} s_{k}\right)=C_{1}$ and $\nu_{\mathscr{B}}\left(s_{j} s_{k}\right)=C_{2}$ for all $s_{j} s_{k} \in$ $E$. By using the definition of vertex degree $(\mathscr{D})_{G}\left(s_{j}\right)=$ $\left((\mathscr{D})_{\mu}\left(s_{j}\right),(\mathscr{D})_{\nu}\left(s_{j}\right)\right)$, we must have

$$
\begin{aligned}
(\mathscr{D})_{\mu}\left(s_{j}\right) & =\sum_{s_{j}, s_{k} \neq s_{j} \in \mathscr{V}} \frac{\mu_{\mathscr{A}}\left(s_{j}\right) \mu_{\mathscr{A}}\left(s_{k}\right)}{\mu_{\mathscr{A}}\left(s_{j}\right)+\mu_{\mathscr{A}}\left(s_{k}\right)-\mu_{\mathscr{A}}\left(s_{j}\right) \mu_{\mathscr{A}}\left(s_{k}\right)} \\
& =\sum_{s_{j} s_{k} \in E} \mu_{\mathscr{B}}\left(s_{j} s_{k}\right)=\sum_{s_{j} s_{k} \in E} C_{1}=R C_{1}=\mathscr{R}_{1}, \\
(\mathscr{D})_{v}\left(s_{j}\right) & =\sum_{s_{j}, s_{k} \neq s_{j} \in \mathscr{V}} \frac{v_{\mathscr{A}}\left(s_{j}\right)+v_{\mathscr{A}}\left(s_{k}\right)-2 v_{\mathscr{A}}\left(s_{j}\right) v_{\mathscr{A}}\left(s_{k}\right)}{1-v_{\mathscr{A}}\left(s_{j}\right) v_{\mathscr{A}}\left(s_{k}\right)} \\
& =\sum_{s_{j} s_{k} \in E} v_{\mathscr{B}}\left(s_{j} s_{k}\right)=\sum_{s_{j} s_{k} \in E} C_{2}=R C_{2}=\mathscr{R}_{2} .
\end{aligned}
$$

Therefore, $G=(\mathscr{A}, \mathscr{B})$ is a $\left(\mathscr{R}_{1}, \mathscr{R}_{2}\right)$-regular PDFG.

Further, by definition of total degree of edge, we have

$$
\begin{aligned}
(\mathscr{T} \mathscr{D})_{\mu}\left(s_{j} s_{k}\right)= & \sum_{s_{j} s_{l} \in E, l \neq k} \mu_{\mathscr{B}}\left(s_{j} s_{l}\right) \\
& +\sum_{s_{k} s_{l} \in E, l \neq j} \mu_{\mathscr{B}}\left(s_{k} s_{l}\right)+\mu_{\mathscr{B}}\left(s_{j} s_{k}\right) \\
= & \sum_{s_{j} s_{l} \in E} C_{1}+\sum_{s_{k} s_{l} \in E} C_{1}+\mathrm{C}_{1} \\
= & C_{1}(R-1)+C_{1}(R-1)+C_{1} \\
= & C_{1}(2 R-1)=\mathrm{K}_{1} \text { for all } s_{j} s_{k} \in E .
\end{aligned}
$$

In the similar manner, we can easily show that $(\mathscr{T} \mathscr{D})_{v}\left(s_{j} s_{k}\right)$ $=\mathrm{K}_{2}$ for all $s_{j} s_{k} \in E$. Hence we conclude that $G=(\mathscr{A}, \mathscr{B})$ is both $\left(\mathscr{R}_{1}, \mathscr{R}_{2}\right)$-regular and $\left(\mathrm{K}_{1}, \mathrm{~K}_{2}\right)$-totally edge regular PDFG.

Conversely, assume that $G$ is both $\left(\mathscr{R}_{1}, \mathscr{R}_{2}\right)$-regular and $\left(\mathrm{K}_{1}, \mathrm{~K}_{2}\right)$-totally edge regular PDFG. To prove that $\mu_{\mathscr{B}}$ and $v_{\mathscr{B}}$ are constant functions, consider the definition of total degree of edge, we have

$$
\begin{aligned}
(\mathscr{T} \mathscr{D})_{\mu}\left(s_{j} s_{k}\right)= & (\mathscr{D})_{\mu_{\mathscr{A}}}\left(s_{j}\right)+(\mathscr{D})_{\mu_{\mathscr{A}}}\left(s_{k}\right) \\
& -\frac{\mu_{\mathscr{A}}\left(s_{j}\right) \mu_{\mathscr{A}}\left(s_{k}\right)}{\mu_{\mathscr{A}}\left(s_{j}\right)+\mu_{\mathscr{A}}\left(s_{k}\right)-\mu_{\mathscr{A}}\left(s_{j}\right) \mu_{\mathscr{A}}\left(s_{k}\right)} \\
\mathrm{K}_{1}= & \mathscr{R}_{1}+\mathscr{R}_{1} \\
& -\frac{\mu_{\mathscr{A}}\left(s_{j}\right) \mu_{\mathscr{A}}\left(s_{k}\right)}{\mu_{\mathscr{A}}\left(s_{j}\right)+\mu_{\mathscr{A}}\left(s_{k}\right)-\mu_{\mathscr{A}}\left(s_{j}\right) \mu_{\mathscr{A}}\left(s_{k}\right)} \\
\mu_{\mathscr{B}}\left(s_{j} s_{k}\right)= & 2 \mathscr{R}_{1}-\mathrm{K}_{1} \text { for all } s_{j} s_{k} \in E .
\end{aligned}
$$

In the similar manner, we can easily show that $\mu_{\mathscr{B}}\left(s_{j} s_{k}\right)=$ $2 \mathscr{R}_{2}-\mathrm{K}_{2}$ for all $s_{j} s_{k} \in E$. Hence $\mu_{\mathscr{B}}$ and $v_{\mathscr{B}}$ are constant functions.

Theorem 17 Let $G=(\mathscr{A}, \mathscr{B})$ be a PDFG on a crisp graph $G^{*}=(\mathscr{V}, E)$. If $\mu_{\mathscr{B}}$ and $\nu_{\mathscr{B}}$ are constant functions, then $G$ 
is $\left(\mathrm{R}_{1}, \mathrm{R}_{2}\right)$-edge regular if and only if $G^{*}$ is an edge regular graph.

Proof Suppose that $\mu_{\mathscr{B}}$ and $\nu_{\mathscr{B}}$ are constant functions such that $\mu_{\mathscr{B}}\left(s_{j} s_{k}\right)=C_{1}$ and $\nu_{\mathscr{B}}\left(s_{j} s_{k}\right)=C_{2}$ for all $s_{j} s_{k} \in E$. Assume that $G$ is $\left(\mathrm{R}_{1}, \mathrm{R}_{2}\right)$-edge regular. To prove that $G^{*}$ is an edge regular graph, we suppose on contrary that $G^{*}$ is not an edge regular graph, i.e., $(\mathscr{D})_{G^{*}}\left(s_{j} s_{k}\right) \neq(\mathscr{D})_{G^{*}}\left(s_{l} s_{n}\right)$ for at least one pair of $s_{j} s_{k}, s_{l} s_{n} \in E$. By definition of degree of edge of PDFG, we have

$$
\begin{aligned}
(\mathscr{D})_{\mu}\left(s_{j} s_{k}\right)= & (\mathscr{D})_{\mu_{\mathscr{A}}}\left(s_{j}\right)+(\mathscr{D})_{\mu_{\mathscr{A}}}\left(s_{k}\right) \\
& -2\left(\frac{\mu_{\mathscr{A}}\left(s_{j}\right) \mu_{\mathscr{A}}\left(s_{k}\right)}{\mu_{\mathscr{A}}\left(s_{j}\right)+\mu_{\mathscr{A}}\left(s_{k}\right)-\mu_{\mathscr{A}}\left(s_{j}\right) \mu_{\mathscr{A}}\left(s_{k}\right)}\right) \\
= & \sum_{s_{j} s_{l} \in E, l \neq k} \mu_{\mathscr{B}}\left(s_{j} s_{l}\right) \\
& +\sum_{s_{k} s_{l} \in E, l \neq j} \mu_{\mathscr{B}}\left(s_{k} s_{l}\right)-2 \mu_{\mathscr{B}}\left(s_{j} s_{k}\right) \\
= & \sum_{s_{j} s_{l} \in E} C_{1}+\sum_{s_{k} s_{l} \in E} C_{1}-2 \mathrm{C}_{1} \\
= & (\mathscr{D})_{G^{*}}\left(s_{j}\right) C_{1}+(\mathscr{D})_{G^{*}}\left(s_{k}\right) C_{1}-2 \mathrm{C}_{1} \\
= & C_{1}\left((\mathscr{D})_{G^{*}}\left(s_{j}\right)+(\mathscr{D})_{G^{*}}\left(s_{k}\right)-2\right) \\
= & C_{1}(\mathscr{D})_{G^{*}}\left(s_{j} s_{k}\right) .
\end{aligned}
$$

Likewise, we can easily show that $(\mathscr{D})_{v}\left(s_{j} s_{k}\right)=C_{2}(\mathscr{D})_{G^{*}}$ $\left(s_{j} s_{k}\right)$ for all $s_{j} s_{k} \in E$. Therefore, $(\mathscr{D})_{G}\left(s_{j} s_{k}\right)=\left(C_{1}(\mathscr{D})_{G^{*}}\right.$ $\left.\left(s_{j} s_{k}\right), C_{2}(\mathscr{D})_{G^{*}}\left(s_{j} s_{k}\right)\right)$ and $(\mathscr{D})_{G}\left(s_{l} s_{n}\right)=\left(C_{1}(\mathscr{D})_{G^{*}}\left(s_{l} s_{n}\right)\right.$, $\left.C_{2}(\mathscr{D})_{G^{*}}\left(s_{l} s_{n}\right)\right)$. As $(\mathscr{D})_{G^{*}}\left(s_{j} s_{k}\right) \neq(\mathscr{D})_{G^{*}}\left(s_{l} s_{n}\right)$, thus $(\mathscr{D})_{G}\left(s_{j} s_{k}\right) \neq(\mathscr{D})_{G}\left(s_{l} s_{n}\right)$, Therefore, $G$ is not $\left(\mathrm{R}_{1}, \mathrm{R}_{2}\right)$ edge regular PDFG, a contradiction. Hence we conclude that $G^{*}$ is an edge regular graph.

Conversely, assume that $G^{*}$ is an edge regular graph. To show that $G$ is $\left(\mathrm{R}_{1}, \mathrm{R}_{2}\right)$-edge regular PDFG, we suppose on the contrary that $G$ is not $\left(\mathrm{R}_{1}, \mathrm{R}_{2}\right)$-edge regular PDFG, i.e., $(\mathscr{D})_{G}\left(s_{j} s_{k}\right) \neq(\mathscr{D})_{G}\left(s_{l} s_{n}\right)$ for at least one pair of $s_{j} s_{k}, s_{l} s_{n} \in$ $E,\left((\mathscr{D})_{\mu}\left(s_{j} s_{k}\right),(\mathscr{D})_{\nu}\left(s_{j} s_{k}\right)\right) \neq\left((\mathscr{D})_{\mu}\left(s_{l} s_{n}\right),(\mathscr{D})_{\nu}\left(s_{l} s_{n}\right)\right)$.

Now $(\mathscr{D})_{\mu}\left(s_{j} s_{k}\right) \neq(\mathscr{D})_{\mu}\left(s_{l} s_{n}\right)$ implies that

$$
\begin{aligned}
& \sum_{s_{j} s_{p} \in E, p \neq k} \mu_{\mathscr{B}}\left(s_{j} s_{p}\right)+\sum_{s_{k} s_{p} \in E, p \neq j} \mu_{\mathscr{B}}\left(s_{k} s_{p}\right) \\
& \quad \neq \sum_{s_{l} s_{m} \in E, m \neq n} \mu_{\mathscr{B}}\left(s_{l} s_{m}\right)+\sum_{s_{n} s_{m} \in E, m \neq l} \mu_{\mathscr{B}}\left(s_{n} s_{m}\right) .
\end{aligned}
$$

As $\mu_{\mathscr{B}}$ is a constant function, we have $(\mathscr{D})_{G^{*}}\left(s_{j} s_{k}\right) \neq$ $(\mathscr{D})_{G^{*}}\left(s_{l} s_{n}\right)$, a contradiction. Hence we conclude that $G$ is a $\left(\mathrm{R}_{1}, \mathrm{R}_{2}\right)$-edge regular PDFG.

\section{Numerical example to decision-making}

In this section, a decision-making problem concerning the evaluation of appropriate ETL (Extract, Transform and Load) software for a Business Intelligence (BI) project (adopted from [47]) is solved to illustrate the applicability of the proposed concept of PDFGs in realistic scenario. The algorithm for the evaluation of appropriate ETL software for a BI project within the framework of Pythagorean fuzzy preference relation (PFPR) [27] is outlined in Algorithm 1.

\section{Evaluation of appropriate ETL software for a BI project}

Business Intelligence, a field of information systems architecture, allows implementation that includes collection, transformation and restoring of data for assisting the decisionmaking experts in enterprises. The central part of BI is established on data warehouses powered by ETL. With the gradual development of BI usage, ETL, the initial point of the project, has become a key factor that affects the failure or success of the BI project. The main task of BI project is the evaluation of most appropriate and suitable ETL software which maximizes the profits, limits the costs, performs well and is flexible to accommodate future advancements in the project. A number of ETL software are available in the market. Each software has its own technique for extracting, loading and transforming of data. A decision-making expert is hired that pairwise compares the five ETL softwares $\mathscr{S}_{l}(l=1,2, \ldots, 5)$ for a new BI project on the basis of the criterion 'functionality and reliability' and provides his preference information in the form of PFPR $\mathscr{Q}=\left(q_{l p}\right)_{5 \times 5}$, where $q_{l p}=\left(\mu_{l p}, v_{l p}\right)$ is the Pythagorean fuzzy element assigned by the decision-making expert with $\mu_{l p}$ as the degree to which the ETL software $\mathscr{S}_{l}$ is preferred over the ETL software $\mathscr{S}_{p}$ with respect to the given criterion and $v_{l p}$ as the degree to which the ETL software $\mathscr{S}_{l}$ is not preferred over the ETL software $\mathscr{S}_{p}$ with respect to the given criterion. The PFPR $\mathscr{Q}=\left(q_{l p}\right)_{5 \times 5}$ is expressed in the following tabular form (Table 1).

The Pythagorean fuzzy directed network $\mathscr{D}$ corresponding to PFPR $\mathscr{Q}$ given in Table 1 , is presented in Fig. 20.

In order to compute the clumped PFE $q_{l p}=\left(\mu_{l p}, v_{l p}\right)$ $(l, p=1,2, \ldots, 5)$ of the ETL software $\mathscr{S}_{l}$, over all the other ETL softwares, we utilize Pythagorean Dombi

Table 1 PFPR of the decision-making expert

\begin{tabular}{llllll}
\hline $\mathscr{Q}$ & $\mathscr{S}_{1}$ & $\mathscr{S}_{2}$ & $\mathscr{S}_{3}$ & $\mathscr{S}_{4}$ & $\mathscr{S}_{5}$ \\
\hline $\mathscr{S}_{1}$ & $(0.5,0.5)$ & $(0.7,0.5)$ & $(0.5,0.6)$ & $(0.3,0.8)$ & $(0.4,0.3)$ \\
$\mathscr{S}_{2}$ & $(0.5,0.7)$ & $(0.5,0.5)$ & $(0.2,0.9)$ & $(0.8,0.4)$ & $(0.1,0.8)$ \\
$\mathscr{S}_{3}$ & $(0.6,0.5)$ & $(0.9,0.2)$ & $(0.5,0.5)$ & $(0.6,0.6)$ & $(0.5,0.4)$ \\
$\mathscr{S}_{4}$ & $(0.8,0.3)$ & $(0.4,0.8)$ & $(0.6,0.6)$ & $(0.5,0.5)$ & $(0.6,0.7)$ \\
$\mathscr{S}_{5}$ & $(0.3,0.4)$ & $(0.8,0.1)$ & $(0.4,0.5)$ & $(0.7,0.6)$ & $(0.5,0.5)$ \\
\hline
\end{tabular}




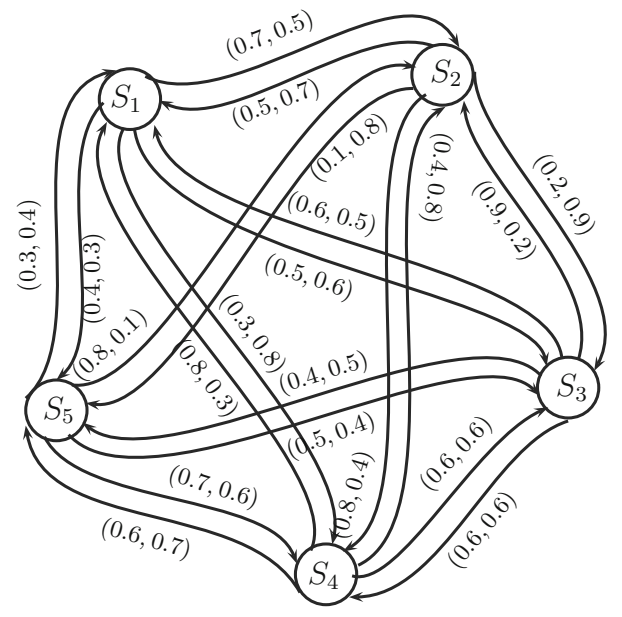

Fig. 20 Directed network of PFPR

fuzzy arithmetic averaging (PDFAA) operator [48] given in Equation 1

$$
\begin{aligned}
& q_{l}=\operatorname{PDFAA}\left(q_{l 1}, q_{l 2}, \ldots, q_{l n}\right) \\
& =\int \sqrt{1-\frac{1}{1+\left[\sum_{p=1}^{n} \frac{1}{n}\left(\frac{\mu_{l p}^{2}}{1-\mu_{l p}^{2}}\right)^{\gamma}\right]^{\frac{1}{\gamma}}}}, \\
& \frac{1}{1+\left[\sum_{p=1}^{n} \frac{1}{n}\left(\frac{1-v_{l p}}{v_{l p}}\right)^{\gamma}\right]^{\frac{1}{\gamma}}} \text {. }
\end{aligned}
$$

As in Dombi's t-norm and t-conorm, we have taken $\gamma=1$. Therefore, in Equation $1, \gamma=1$ is considered to obtain the combined overall preference value $q_{l}(l=1,2, \ldots, 5)$, which is given below:

$$
\begin{aligned}
& q_{1}=(0.5264,0.4878), q_{2}=(0.5770,0.6032), \\
& q_{3}=(0.7401,0.3798), \\
& q_{4}=(0.6377,0.5166), q_{5}=(0.6341,0.2752) .
\end{aligned}
$$

The score functions $\mathbb{S}\left(q_{l}\right)$ of the combined overall preference value $q_{l}(l=1,2, \ldots, 5)$ is calculated by utilizing $\mathbb{S}\left(q_{l}\right)=\mu_{l}^{2}-v_{l}^{2}[16]$, which is shown below:

$\mathbb{S}\left(q_{1}\right)=0.0386, \quad \mathbb{S}\left(q_{2}\right)=-0.0262$,

$\mathbb{S}\left(q_{3}\right)=0.3603, \quad \mathbb{S}\left(q_{4}\right)=0.1211$ and $\mathbb{S}\left(q_{5}\right)=0.3589$.
On the basis of score functions, we get the ranking of the ETL softwares $\mathscr{S}_{l}, l=1,2, \ldots, 5$ as follows:

$\mathscr{S}_{3} \succ \mathscr{S}_{5} \succ \mathscr{S}_{4} \succ \mathscr{S}_{1} \succ \mathscr{S}_{2}$.

According to the ranking, it is concluded that $\mathscr{S}_{3}$ is the most appropriate ETL software for a new project among all.

If Pythagorean Dombi fuzzy geometric averaging (PDFGA) operator [48] is applied instead of PDFAA operator, then the clumped PFE $q_{l p}=\left(\mu_{l p}, v_{l p}\right)(l, p=$ $1,2, \ldots, 5)$ of the ETL software $\mathscr{S}_{l}$, over all the other ETL softwares, is obtained by using Eq. 2 :

$$
\begin{aligned}
q_{l} & =\left(\frac{\operatorname{PDFGA}\left(q_{l 1}, q_{l 2}, \ldots, q_{l n}\right)}{1+\left[\sum_{p=1}^{n} \frac{1}{n}\left(\frac{1-\mu_{l p}}{\mu_{l p}}\right)^{\gamma}\right]^{\frac{1}{\gamma}}},\right. \\
& \left.\sqrt{1-\frac{1}{1+\left[\sum_{p=1}^{n} \frac{1}{n}\left(\frac{v_{l p}^{2}}{1-v_{l p}^{2}}\right)^{\gamma}\right]^{\frac{1}{\gamma}}}}\right) .
\end{aligned}
$$

For $\gamma=1$, we obtain

$q_{1}=(0.4440,0.6190), q_{2}=(0.2469,0.7751)$,

$q_{3}=(0.5921,0.4756)$,

$q_{4}=(0.5504,0.6538), q_{5}=(0.4756,0.4715)$.

The score functions $\mathbb{S}\left(q_{l}\right)$ of the combined overall preference value $q_{l}(l=1,2, \ldots, 5)$ is calculated by utilizing $\mathbb{S}\left(q_{l}\right)=$ $\mu_{l}^{2}-v_{l}^{2}[16]$, which is shown below:

$\mathbb{S}\left(q_{1}\right)=-0.1750, \quad \mathbb{S}\left(q_{2}\right)=-0.5282$,

$\mathbb{S}\left(q_{3}\right)=0.1165, \quad \mathbb{S}\left(q_{4}\right)=-0.1034$ and $\mathbb{S}\left(q_{5}\right)=0.0041$.

On the basis of score functions, we get the ranking of the ETL softwares $\mathscr{S}_{l}, l=1,2, \ldots, 5$ as follows:

$\mathscr{S}_{3} \succ \mathscr{S}_{5} \succ \mathscr{S}_{4} \succ \mathscr{S}_{1} \succ \mathscr{S}_{2}$.

According to the ranking, it is concluded that $\mathscr{S}_{3}$ is the most appropriate ETL software for a new project among all

We present our proposed method for decision-making in the following Algorithm 1: 


\section{$\overline{\text { Algorithm } 1 \text { The algorithm for evaluation of appropriate }}$ \\ ETL software for a BI project.}

INPUT: A discrete set of feasible alternatives $\mathscr{S}=\left\{\mathscr{S}_{1}, \mathscr{S}_{2}, \ldots, \mathscr{S}_{n}\right\}$, a conflicting criterion in order to achieve the target and construction of PFPR $\mathscr{Q}=\left(q_{l p}\right)_{n \times n}$ corresponding to considered criterion.

OUTPUT: The selection of optimal alternative.

1. begin

2. Aggregate all $q_{l p}=\left(\mu_{l p}, \nu_{l p}\right)(l, p=1,2, \ldots, n)$ corresponding to the alternative $\mathscr{S}_{l}$ and get the PFE $q_{l}$ of the alternative $\mathscr{S}_{l}$ over all the other alternatives by utilizing the PDFAA operator

$$
\begin{aligned}
& q_{l}=\operatorname{PDFAA}\left(q_{l 1}, q_{l 2}, \ldots, q_{l n}\right) \\
& =\left(\sqrt{1-\frac{1}{1+\left[\sum_{p=1}^{n} \frac{1}{n}\left(\frac{\mu_{l p}^{2}}{1-\mu_{l p}^{2}}\right)^{\gamma}\right]^{\frac{1}{\gamma}}}},\right. \\
& \left.\frac{1}{1}\right), \quad l=1,2,3, \ldots, n \text {. } \\
& 1+\left[\sum_{p=1}^{n} \frac{1}{n}\left(\frac{1-v_{l p}}{v_{l p}}\right)^{\gamma}\right] \bar{\gamma}
\end{aligned}
$$

or PDFGA operator

$$
\begin{aligned}
& q_{l}=\operatorname{PDFGA}\left(q_{l 1}, q_{l 2}, \ldots, q_{l n}\right) \\
& =\left(\frac{1}{1+\left[\sum_{p=1}^{n} \frac{1}{n}\left(\frac{1-\mu_{l p}}{\mu_{l p}}\right)^{\gamma}\right]^{\frac{1}{\gamma}}},\right. \\
& \sqrt{\left.1-\frac{1}{1+\left[\sum_{p=1}^{n} \frac{1}{n}\left(\frac{v_{l p}^{2}}{1-v_{l p}^{2}}\right)^{\gamma}\right]^{\frac{1}{\gamma}}}\right)}, \quad l=1,2,3, \ldots, n \text {. }
\end{aligned}
$$

3. Compute the score functions $\mathbb{S}\left(q_{l}\right)$ of the combined overall preference value $q_{l}(l=1,2, \ldots, n)$ by using $\mathbb{S}\left(q_{l}\right)=\mu_{l}^{2}-v_{l}^{2}$.

4. On the basis of score functions $\mathbb{S}\left(q_{l}\right)(l=1,2, \ldots, n)$, rank all the alternatives $\mathscr{S}_{l}(l=1,2, \ldots, n)$.

5. Output the optimal alternative.

6. end

The results computed in this section are compared (given in Table 2) with the decision results in [47], where the problem related to evaluation of ETL software is solved by AHP-TOPSIS method. Table 2 exhibits that the decision results of [47] are consistent with our proposed PDFAA and PDFGA method, which interprets the authenticity of the method.

Table 2 The decision results of the alternatives using the different methods

\begin{tabular}{ll}
\hline Methods & Ranking of alternatives \\
\hline Hanine et al. [47] & $\mathscr{S}_{3} \succ \mathscr{S}_{4} \succ \mathscr{I}_{5} \succ \mathscr{S}_{2} \succ \mathscr{S}_{1}$ \\
Our proposed PDFAA method & $\mathscr{S}_{3} \succ \mathscr{S}_{5} \succ \mathscr{S}_{4} \succ \mathscr{S}_{1} \succ \mathscr{S}_{2}$ \\
Our proposed PDFGA method & $\mathscr{S}_{3} \succ \mathscr{S}_{5} \succ \mathscr{S}_{4} \succ \mathscr{S}_{1} \succ \mathscr{S}_{2}$ \\
\hline
\end{tabular}

\section{Conclusions}

Graphs are the wide-ranging models found almost everywhere in the human made and natural structures such as, for modeling+ relationships and process dynamics in social, biological and physical systems. Pythagorean fuzzy models are more practical and useful than fuzzy and intuitionistic fuzzy models as it provide additional spaces between membership and non-membership grades, for representing imprecise and incomplete information which occur in real world scenarios. In this paper, we have observed the excellent flexibility of Dombi operators with operational parameters in graph theoretical concepts under Pythagorean fuzzy environment. Further, we have inspected some substantial characteristics like strongness, completeness, vertex and edge regularity. As vertex and edge regularity, particularly regular, totally regular, strongly regular and biregular graphs are extensively applied in designing reliable computer networks and matrix representations, they led many developments in structural theory of graphs. Some necessary and sufficient conditions have been initiated to justify above indicated characteristics. By taking distinct values of operational parameters, many decision-making problems can be easily handled. Our work has adopted an incentive approach towards a decisionmaking problem concerning the evaluation of appropriate ETL software for a business intelligence project. Further work will pay particular attention on (1) Interval-valued Pythagorean fuzzy graphs; (2) Simplified interval-valued Pythagorean fuzzy graphs and (3) Hesitant Pythagorean fuzzy graphs.

\section{Compliance with ethical standards}

Conflict of interest The authors declare that they have no conflict of interest regarding the publication of the research article.

Open Access This article is distributed under the terms of the Creative Commons Attribution 4.0 International License (http://creativecomm ons.org/licenses/by/4.0/), which permits unrestricted use, distribution, and reproduction in any medium, provided you give appropriate credit to the original author(s) and the source, provide a link to the Creative Commons license, and indicate if changes were made.

\section{References}

1. Zadeh LA (1965) Fuzzy sets. Inf Control 8(3):338-353

2. Hamacher H (1978) On logical aggregations of non-binar explicit decision criteria. Rita G. Fischer Verlag, Frankfurt

3. Kuwagaki A (1952) On the rational functional equation of function unknown of two variables. Memoirs of the College of Science 28(2)

4. Menger K (1942) Statistical metrics. J Natl Acad Sci 28(12):535537

5. Schweizer B, Sklar S (1983) Probabilistic metric spaces. Probability and Applied Mathematics 
6. Alsina C, Trillas E, Valverde L (1983) On some logical connectives for fuzzy sets theory. J Math Anal Appl 93(1):15-26

7. Klement PE, Mesiar R, Pap E (2000) Triangular norms. Kluwer Academic, Dordrecht

8. Hamacher H (1978) Logical combinations of fuzzy statements and their relative valuation functions. Cybern Syst Res 3:276-288

9. Dubois D, Ostasiewicz W, Prade H (2000) Fuzzy sets: history and basic notions. Handbook of fuzzy sets and possibility theory. Springer, New York, pp 121-124

10. Rosenfeld A (1975) Fuzzy graphs. In: Zadeh LA, Fu KS, Shimura $\mathrm{M}$ (eds) Fuzzy sets and their applications to cognitive and decision processes. Academic Press, Cambridge, pp 77-95

11. Atanassov KT (1986) Intuitionistic fuzzy sets. Fuzzy Sets Syst 20(1):87-96

12. Shannon A, Atanassov KT (1994) A first step to a theory of intuitioistic fuzzy graphs. In: Lakov D (ed) Proceedings of Fuzzy Based Expert Systems. Sofia, pp 59-61

13. Yager RR (2013) Pythagorean fuzzy subsets. Joint IFSA World Congress and NAFIPS Annual Meeting, pp 57-61

14. Yager RR, Abbasov AM (2013) Pythagorean membership grades, complex numbers and decision making. Int J Intell Syst 28(5):436452

15. Yager RR (2014) Pythagorean membership grades in multi-criteria decision making. IEEE Trans Fuzzy Syst 22(4):958-965

16. Zhang X, Xu Z (2014) Extension of TOPSIS to multiple criteria decision making with Pythagorean fuzzy sets. Int J Intell Syst 29(12):1061-1078

17. Peng X, Yang Y (2015) Some results for Pythagorean fuzzy sets. Int J Intell Syst 30(11):1133-1160

18. Ren P, Xu Z, Gou X (2016) Pythagorean fuzzy TODIM approach to multi-criteria decision making. Appl Soft Comput 42:246-259

19. Akram M, Dudek WA, Ilyas F (2019) Group decision-making based on pythagorean fuzzy TOPSIS method. International Journal of Intelligent Systems. https://doi.org/10.1002/int.22103

20. Rangasamy P, Palaniappan N (2003) Some operations on intuitionistic fuzzy sets of second type. Intuitionistic Fuzzy Sets 10(2):1-19

21. Peng X, Selvachandran G (2017) Pythagorean fuzzy set: state of the art and future directions. Artifical Intelligence (Artif Intell Rev) https://doi.org/10.1007/s10462-017-9596-9

22. Garg H (2018) A new exponential operational laws and their aggregation operators of interval-valued Pythagorean fuzzy information. Int J Intell Syst 33(3):653-683

23. Garg H (2018) Some methods for strategic decision-making problems with immediate probabilities in Pythagorean fuzzy environment. Int J Intell Syst 33(4):687-712

24. Garg H (2018) Linguistic Pythagorean fuzzy sets and its applications in multi attribute decision making process. Int J Intell Syst 33(6):1234-1263

25. Garg H (2018) Hesitant Pythagorean fuzzy sets and their aggregation operators in multiple-attribute decision-making. Int J Uncertain Quantif 8(3):267-289

26. Garg H (2016) A novel correlation coefficients between Pythagorean fuzzy sets and its applications to decision-making processes. Int J Intell Syst 31(12):1234-1253

27. Naz S, Ashraf S, Akram M (2018) A novel approach to decisionmaking with Pythagorean fuzzy information. Mathematics 6(6):95. https://doi.org/10.3390/math6060095

28. Verma R, Merigo JM, Sahni M Pythagorean fuzzy graphs: some results. arXiv:1806.06721v1

29. Akram M, Naz S (2018) Energy of Pythagorean fuzzy graphs with applications. Mathematics 6(8):136. https://doi.org/10.3390/ math6080136

30. Akram M, Habib A, Ilyas F, Dar, JM (2018) Specific types of Pythagorean fyzzy graphs and application to decision-making.
Mathematical and Computational Application 23(42). https://doi. org/10.3390/mca23030042.

31. Akram M, Dar JM, Naz S (2019) Certain graphs under pythagorean fuzzy environment. Complex and Intelligent Systems 1-18. https:// doi.org/10.1007/s40747-018-0089-5

32. Akram M, Dar JM, Farooq A (2018) Planar graphs under Pythagorean fuzzy environment. Mathematics 6(12):278. https:// doi.org/10.3390/math6120278

33. Akram M, Habib A (2019) $q$-Rung picture fuzzy graphs: a creative view on regularity with applications. J Appl Math Comput. https:// doi.org/10.1007/s12190-019-01249-y

34. Habib A, Akram M, Farooq A (2019) q-rung orthopair fuzzy competition graphs with application in the soil Ecosystem. Mathematics 7(1) https://doi.org/10.3390/math7010091

35. Dombi J (1982) A general class of fuzzy operators, the De Morgan class of fuzzy operators and fuzziness measures induced by fuzzy operators. Fuzzy Sets Syst 8(2):149-163

36. Chen J, Ye J (2017) Some Single-Valued Neutrosophic Dombi weighted aggregation operators for multiple attribute decisionmaking. Symmetry 9(6). https://doi.org/10.3390/sym9060082

37. Jana C, Pal M, Wang J (2018) Bipolar fuzzy Dombi aggregation operators and its application in multiple-attribute decision-making process. J Ambient Intell Hum Comput 1-17

38. Shi L, Ye J (2018) Dombi Aggregation operators of neutrosophic cubic sets for multiple attribute decision-making. Algorithms 11(29). https://doi.org/10.3390/a11030029.

39. Liu PD, Liu JL, Chen SM (2018) Some intuitionistic fuzzy Dombi Bonferroni mean operators and their application to multi-attribute group decision making. J Oper Res Soc 69(1):1-24

40. He X (2018) Typhoon disaster assessment based on Dombi hesitant fuzzy information aggregation operators. Nat Haz 90(3):11531175

41. Ashraf S, Naz S, Kerre EE (2018) Dombi fuzzy graphs. Fuzzy Inf Eng 10(1):58-79

42. Harary F (1972) Graph theory, 3rd edn. Addison-Wesley, Reading

43. Nagoor Gani A, Radha K (2008) On regular fuzzy graphs. J Phys Sci 12:33-44

44. Radha K, Kumaravel N (2014) On edge regular fuzzy graphs. Int J Math Arch 5(9):100-112

45. Radha K, Rosemine A (2017) Strongly regular fuzzy graph. International Journal of Scintific and Reasearch Publications 7(7)

46. Karunambogai MG, Sivasankar S, Palanivel K (2015) Some properties of a regular intuitionistic fuzzy graph. Int J Math Comput 26(4):53-61

47. Luqman A, Akram M, Al-Kenani N (2019) $q$-rung orthopair fuzzy hypergraphs with applications. Mathematics 7(3):260. https://doi. org/10.3390/math7030260

48. Akram M, Habib A, Koam AN (2019) A novel description on edgeregular $q$-rung picture fuzzy graphs with application. Symmetry 11:489. https://doi.org/10.3390/sym11040489

Publisher's Note Springer Nature remains neutral with regard to jurisdictional claims in published maps and institutional affiliations. 